$\frac{\text { WASH }-736}{\text { BIOLOGY AND MEDICINE }}$
(TD-4500, 13th ed.)

Distributed: September 27, 1957

\author{
PROCEEDINGS OF THE SECOND ANNUAL MEETING \\ ON \\ BIO-ASSAY AND ANALYTICAL CHEMISTRY \\ October 11 and 12, 1956
}

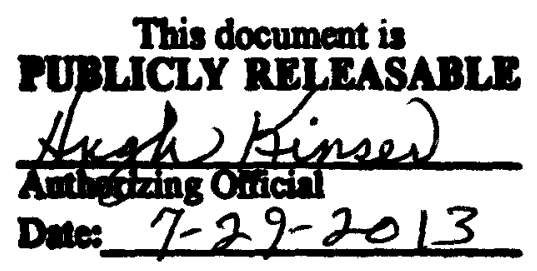

$-1+2$

Sponsored by

Industrial Hygiene Laboratory, Health Division

Los Alamos Scientific Laboratory

University of California

Los Alamos, New Mexico 


\section{DISCLAIMER}

This report was prepared as an account of work sponsored by an agency of the United States Government. Neither the United States Government nor any agency Thereof, nor any of their employees, makes any warranty, express or implied, or assumes any legal liability or responsibility for the accuracy, completeness, or usefulness of any information, apparatus, product, or process disclosed, or represents that its use would not infringe privately owned rights. Reference herein to any specific commercial product, process, or service by trade name, trademark, manufacturer, or otherwise does not necessarily constitute or imply its endorsement, recommendation, or favoring by the United States Government or any agency thereof. The views and opinions of authors expressed herein do not necessarily state or reflect those of the United States Government or any agency thereof. 


\section{DISCLAIMER}

Portions of this document may be illegible in electronic image products. Images are produced from the best available original document. 


\section{INTRODUCTION}

The only previous meeting of the type described in this document was held in October 1955 at Fernald, Ohio, under the auspices of the National Lead Company of Ohio; the proceedings of that meeting are described in USAEC NLCO-595.

The primary objective of that meeting, and of the meeting herein described, was the interchange of information between those working in this field of health protection, sometimes called "bio-assay," health radiochemistry, occupational biochemistry, or radiation hygiene chemistry. It is the hope of those who participated in the sponsorship of this meeting that these proceedings will aid in the attainment of that objective. 
CONTENTS

Page

Introduction $\quad 3$

Polonium Determination in Urine, Feces, and Blood - H. E. Meyer 7

Determination of Beryllium in Biological Materials - J. H. Harley 22

Application of Statistical Methods to Bio-assay - J. W. Healy 37

Low-level Alpha Counting and Techniques of Pulse-height

Analysis - R. J. Watts

61

Panel Discussion of Plutonium - W. H. Langham, J. W. Healy,

H. J. Foreman, S. M. Sanders, Jr.

The Fission-product Problem - P. S. Harris 106

The Determination of Iodine-131 and Strontium-90 in Urine -

A. G. Schrodt

Procedure for the Radiochemical Analysis of Strontium and Barium in Human Urine - L. B. Farabee

The Human Counter - E. C. Anderson 


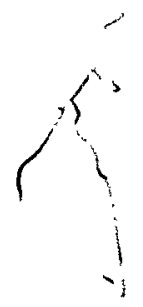


POLONIUM DETERMINATION IN URINE, FECES, AND BLOOD

Herbert E. Meyer

Mound Laboratory

Miamisburg, Ohio

$\underline{\text { Introduction }}$

The estimation of polonium in the body may be conveniently accomplished on a routine basis by the determination of micro-microcurie quantities excreted in the urine. A determination of polonium in the feces and blood may also be made but the time and labor involved compared to that required to do a urinalysis makes feces and blood analysis impractical on a routine basis. The urinalysis is sufficiently sensitive, efficient, and accurate as a polonium body-burden indicator to be used as a routine monitoring control on employees. Feces and blood analyses are used in special cases to assist in estimating body concentrations when the urinalysis has indicated a very high exposure. ${ }^{1}$

\section{Urinalysis}

At the start of the program of developing a suitable urinalysis procedure it was deemed desirable that the procedure should be simple as well as sensitive, efficient, and accurate so that technical personnel could be employed to do the routine analysis of a large number of samples. Several methods of digestion were investigated; however, each of these methods resulted in prohibitive self-absorption of the alpha particles due to the high concentration of salts which crystallized out when the sample mount was made. A tellurium scavenger method was also attempted, but the procedure involved too many chemical manipulations, and difficulties arose in counting the final mount. A direct electrolysis was tried and resulted in a fairly good recovery. This last method was also too time-consuming and required an 
involved electrolytic set-up. Finally, electrochemical deposition of polonium directly on metals from an acidified urine solution was tried. This method proved effective and was developed into the present method for routine analysis.

Personnel working in polonium areas are required to submit spot urine specimens at regularly scheduled intervals depending upon the frequency of contact with polonium, the types of operations performed, and the amounts of polonium handled in the employee's normal work area. Routine work with polonium requires a weekly urine specimen. Samples are submitted at work in half-pint waxed Sealrite food containers, at the convenience of the employee sometime during the morning of the first day of the work week. The sampling period ends at noon. Since the analysis is based on a $50-\mathrm{ml}$ aliquot, a spot sample only is required. At the close of the sampling period the specimen containers are collected and delivered to the urinalysis laboratory for processing.

At the time of assay a 50-ml aliquot of each sample is thoroughly shaken, poured into a waxed paper cup, and the acidity of the solution is brought to $1 \mathrm{~N}$ by the addition of $10 \mathrm{ml}$ of $6 \mathrm{~N}$ hydrochloric acid. A 1-in.diameter copper disc is suspended from a glass hook and submerged in a vertical position in each sample. By means of a multiple unit mechanical stirrer the samples are agitated with glass stirring rods for a 2-hr period. At the end of the plating time the copper discs are removed from the solution, washed in tap water, and dried. In air-conditioned laboratories it has been found that the dises dry quickiy and cleaniy in the air without the use of drying aids such as dipping in alcohol and ether. ${ }^{2}$ The alpha particles emitted by the polonium on each disc are counted on a low-background proportional alpha counter. The sum of the counts on the two sides measures the amount of polonium extracted from the specimen.

During the research and development period of this procedure, the efficiency rate was established from a large number of determinations. It was found that there is a relatively constant efficiency of extraction for concentrations of polonium up to $200 \mathrm{c} / \mathrm{m}$ for $50 \mathrm{ml}$ of urine but a steady decline in efficiency above this count. The mean per cent recovery was 86.1 per cent with a standard deviation of 11.9 per cent. The per cent recovery for the population used did not differ significantly from the normal distribution. Therefore, for routine urine assays it can be expected that 67 per cent of the recoveries will fall between the values 74.2 and 98 per cent. The efficiency of recovery by this procedure depends upon the following factors:

1. If the urine specimen is permitted to stand in the collecting container for any appreciable time before assay, the polonium combines with the precipitate which forms in the urine. This polonium is not recovered by the standard plating procedure, but higher polonium recoveries can be achieved by adding $50 \mathrm{mg}$ of sulfamic acid to the container before it is used. 
2. The metal used for the disc affects the recovery percentage.

3. The disc area is an important factor, in that copper discs of 3/4-in. diameter are only 79 per cent as efficient as those of 1-in. diameter. The latter were found to remove 92 per cent of the polonium in solution in one 3-hr plating period.

4. The most efficient acids were found to be nitric and hydrochloric, and the optimum normality of the urine-hydrochloric solution is between 0.5 and $1.5 \mathrm{~N}$.

5. The optimum plating time for 1-in. copper discs is $3 \mathrm{hr}$ using hydrochloric acid at $1 \mathrm{~N}$, and longer plating periods do not increase the recovery. Higher than usual copper ion concentrations can occur at high acid normalities and long extraction times. These excess copper lons in plating solution will tend to lower the polonium recovery.

6. Tests were conducted to determine the degree of agitation required. Varying the stirrer speeds between 150 and $700 \mathrm{rpm}$ had no apparent effect on the efficiency of extraction.

7. The copper discs used for polonium extraction must be clean and unscratched. Since these discs are stamped out of 16-gauge sheet stock in the machine shop, they are usually solled with oil and grease and are frequently scratched. Discs with large or deep scratches are discarded. The oil and grease, which would also affect the extraction efficiency by masking the copper surface, must be removed. The commonly used cleaning procedure is to dip the discs successively in the following solutions: (1) hot concentrated nitric acid, (2) water bath, (3) concentrated nitric acid, (4) two water baths, (5) concentrated hydrochloric acid, (6) two water baths, (7) two ethyl alcohol baths, and (8) two ether baths. This procedure produces an uncontaminated, clean, bright surface. Ten per cent of the clean discs are counted for a background which is normally less than $0.5 \mathrm{c} / \mathrm{m}$ per disc primarily due to the natural activity of the metal.

In addition to simplicity, the electrochemical deposition procedure has several other merits. It is rapid, consistent, and sensitive. One operator can process many samples daily, depending primarily upon the capacity of the stirring apparatus. The same basic procedure can be used to assay both aqueous samples with a low polonium concentration and digested solid samples.

\section{Feces and Blood Samples}

Feces and blood samples may be analyzed to help estimate a body concentration if the urinalysis has indicated a high exposure. Any employee requested to submit a feces sample is provided with a 1-pint Sealrite container for the purpose of collecting a 24-hr sample. For the analysis 
25 to $50 \mathrm{~g}$ of collected sample are treated by a standard nitric acidperchloric acid digestion procedure. After digestion, methyl violet indicator is added and the solution titrated with 75 per cent sodium hydroxide to a $\mathrm{pH}$ of 1 . Hydrochloric acid of $0.5 \mathrm{~N}$ is added to bring the solution to the desired volume and $50-\mathrm{ml}$ aliquots of the solution are plated on copper discs as in the urinalysis procedure. ${ }^{3}$

If a blood sample is required, the employee is requested to report to the medical department where the plant nurse withdraws $5 \mathrm{ml}$ of blood. The specimen is then sent to the bio-assay laboratory for analysis. The sample is oxalated and $1 \mathrm{ml}$ is treated with a potassium permanganate-sulfuric acid solution and heated in a water bath for $15 \mathrm{~min}$. When the solution clears, water is added to bring the volume to $100 \mathrm{ml}$. The solution is then plated on copper discs as in the urinalysis procedure. This method may not give satisfactory results if the blood specimen is old since old blood may not digest completely. In this case a standard perchloric acid digestion procedure may be used since it will dissolve both new and old blood easily.

In assaying the polonium concentration of peripheral blood in animals used in biological experiments the standard chemical digestion proved to be very time-consuming and laborious. Therefore, a method was developed for direct electronic assay of blood smears. This procedure uses a 5-lambda sample of blood drawn into a calibrated Kato micropipette. The sample is then expelled onto a clean lint-free glass slide, measuring 1 by $1-1 / 2$ in., and is immediately covered by a similar slide. This permits the blood to spread evenly between the two surfaces and forms two smears of the sample when the slides are separated. The slides are allowed to dry before they are counted for alpha particle emission. The sum of the counts of the two slides measures the polonium content of the 5-lambda specimen. For greater accuracy an average of the assays from two 5 lambda samples is used for the recovery figure.

The polonium recoveries by the standard chemical digestion method and by the direct assay method were studied by comparing results obtained from two groups of polonium-injected rats. One group was administered a dose of $36.6 \mu \mathrm{c} / \mathrm{Kg}$ of body weight and the other group $6 \mu \mathrm{c} / \mathrm{Kg}$ of body weight. The average recovery by direct assay was lower for low-level blood concentrations than for high-level blood concentrations. The recoveries show that duplicate samples by the two methods would have equal precision providing a factor relating the two methods was known. However, in a study of polonium blood levels made at various post-injection time intervals, the two methods had equal precision. In such studies the direct assay method saves much time and labor. ${ }^{4}$ 
Variations in Polonium Excretion

In addition to the many analytical problems investigated the physiological problems of diurnal variations in human excretion of polonium in urine were also studied. The following information was obtained: (1) samples passed in the morning and evening have higher concentrations of polonium than those passed in the early afternoon; (2) the concentration of polonium in the second sample passed in the morning was, on the average, lower than the concentration in the first; (3) the concentration in the first sample passed in the morning was higher than the concentration in the 24-hr sample; (4) the concentration over a 24-hr period was rather constant and independent of the volume of urine eliminated; (5) the quantity of polonium eliminated per sample and the concentration in each sample varied during any 24-hr period.

From these results it can be seen that the best estimate of body burden is obtained from a 24-hr sample. However, the routine monitoring of a large group of people by analyzing $24-\mathrm{hr}$ samples is a tremendous burden. No correction factor could be found that could be applied to any given spot sample to obtain the average $24-\mathrm{hr}$ concentration of polonium. Since the concentration in the first sample passed in the morning is generally higher than the concentration in the 24-hr sample, the first sample could be collected as a spot sample and the polonium yield taken as the average of all equal aliquots for the 24-hr elimination. This would place the estimate on the high side and actually introduce a safety factor.

On a routine basis we found a program of obtaining the employee's first specimen of the day at home was ineffective since the employees encountered difficulties too numerous to record. During the past few years it has been the practice for employees to submit a specimen at work sometime during the morning of the assigned sampling day. Should the analyses of a spot sample indicate a polonium concentration in excess of the maximum permissible, a second spot sample is immediately requested. If the second sample verifies the results of the first, the employee is assigned to other work in a cold area until successive spot samples indicate that the average body burden during the period of internal exposure is less than the maximum permissible.

\section{Polonium Excretion}

In the early days of the polonium program the problem of excretion rates was studied at the University of Rochester School of Medicine and Dentistry. The determinations were made on a large group of male rats following the intravenous administration of 1.5 to $30 \mu \mathrm{c}$ of polonium per 
kilogram of body weight. Expressed as the per cent of body content, the daily excretion rate in urine was found to be approximately 0.13 per cent per day after the fortieth day post-injection. The average daily fecal excretion rate was found to be about 0.7 per cent of the body content. The determination of the urinary and fecal excretion rates as per cent of body content permits the simple addition of the loss rates for urine, feces, and by radioactive decay,* to give the total elimination rate of polonium from the body. The total rate of elimination is about 5 per cent of the body content per day on the third day and declines to about 1.3 per cent per day at the eightieth day and thereafter. After 80 days the urinary excretion accounts for about 10 per cent, fecal excretion 53 per cent, and radioactive decay for 37 per cent of the daily loss of polonium from the body. ${ }^{6}$ Data on a limited number of human subjects at the University of Rochester indicated no marked deviation in excretion rate, on the average, from the rat data.

Records on accidentally exposed individuals at the temporary wartime Dayton facilities prior to the construction of Mound Laboratory provided a source of data on the human excretion of polonium by means of the urine. These data were used to obtain a measure of the half-life of polonium in the human body. Although the study here is of interest, the significance is uncertain because of deficiencies in the data. Since urinalysis technique is not without error and the urine sampling method was not adequately controlled, the analysis of the accumulated data must be limited to an estimate. These records were used because more adequately controlled data were not available on humans. In these studies the only cases used were those in which the maximum recorded counts per minute for $50 \mathrm{ml}$ of urine were sufficient to indicate a very positive exposure. The counts included in calculating the half-lives were all those from the first high counts down to, and including, the first two consecutive counts below a count of 12 . In the calculations it was assumed that the human excretion patterns were truly exponential with respect to time and followed the decay formula.

The average half-life obtained in this study was 36.6 days. $^{?}$ By using this derived effective half-life a biological half-life of approximately 50 days may be calculated. From the biological half-life figure we may calculate a total daily body excretion rate of 1.4 per cent. This derived elimination rate compares favorably with the polonium excretion rate determined by animal experimentation with injected single doses. An attempt also was made to correlate the polonium concentration in blood and urine of personnel who had received an exposure. Although the correlation on human subjects was found to be rather poor the concentration in the blood was approximated at 35 times the concentration in the urine. ${ }^{8}$

*Radioactive decay for polonium is 0.5 per cent per day. 
Alpha Counting

One of the most time consuming operations in making a polonium determination of the aqueous solutions, to which all specimens are reduced, is the final operation of obtaining the alpha count of the polonium plated on the copper disc. Since the polonium is plated on the surface of the disc, both sides must be counted in order to obtain a total count. This is taken to be 86 per cent of the polonium which was in the solution. In low counting ranges, such as are expected in routine urinalysis, the counting time per specimen is considerable. This system of counting may be thought of as a two-operation alpha count. The usefulness of a counting instrument which would count both surfaces of the copper disc in one operation was apparent. Accordingly, a single operation alpha counter for plated samples was developed.

To count 50 per cent of the alpha particles in one operation the entire plated surface must be exposed to the counting volume. This was achieved by plating the polonium in solution on $1-1 / 2-i n$. lengths of $5 / 16-i n$. copper tubing which had been spun closed on one end. The plated copper tube in position formed the center electrode in the specially designed ionization chamber of the counter. The charge collected on the center electrode is coupled directly to the input of the preamplifier, the output of which energizes the counter. ${ }^{9}$ Several test runs were made comparing the plated disc and tube. It was concluded that the single-operation counter was capable of giving results at least as good as those obtained from the parallel-plate counter and in half the counting time. The use of the plated tube affords the opportunity of counting plated samples at lower counting rates than are practical with the two-count system. ${ }^{10}$ This counting system has not been taken beyond the prototype and experimental stage. However, it does offer some definite advantages for anyone operating a large scale polonium bio-assay program.

\section{Equipment}

The equipment used to "mass produce" the plating and extraction of polonium from aqueous specimens is very simple. See Fig. 1.

The plating apparatus is built in two tiers holding 24 samples each. Each tier has a drive motor saddled between the two lines of pulleys. The shafts of the pulleys ride on a double set of ball bearings for minimum wear and friction. Endless linen belting of the type used on pantograph machines has been found much more satisfactory than leather belting and has been used for about two years without showing appreciable wear.

Each specimen is placed in a pleated waxed-paper Lily cup inscribed with a 50-ml mark. These marks are made by inserting the cups into a contour-bored, aluminum, cup-marking jig which has a small colored crayon 


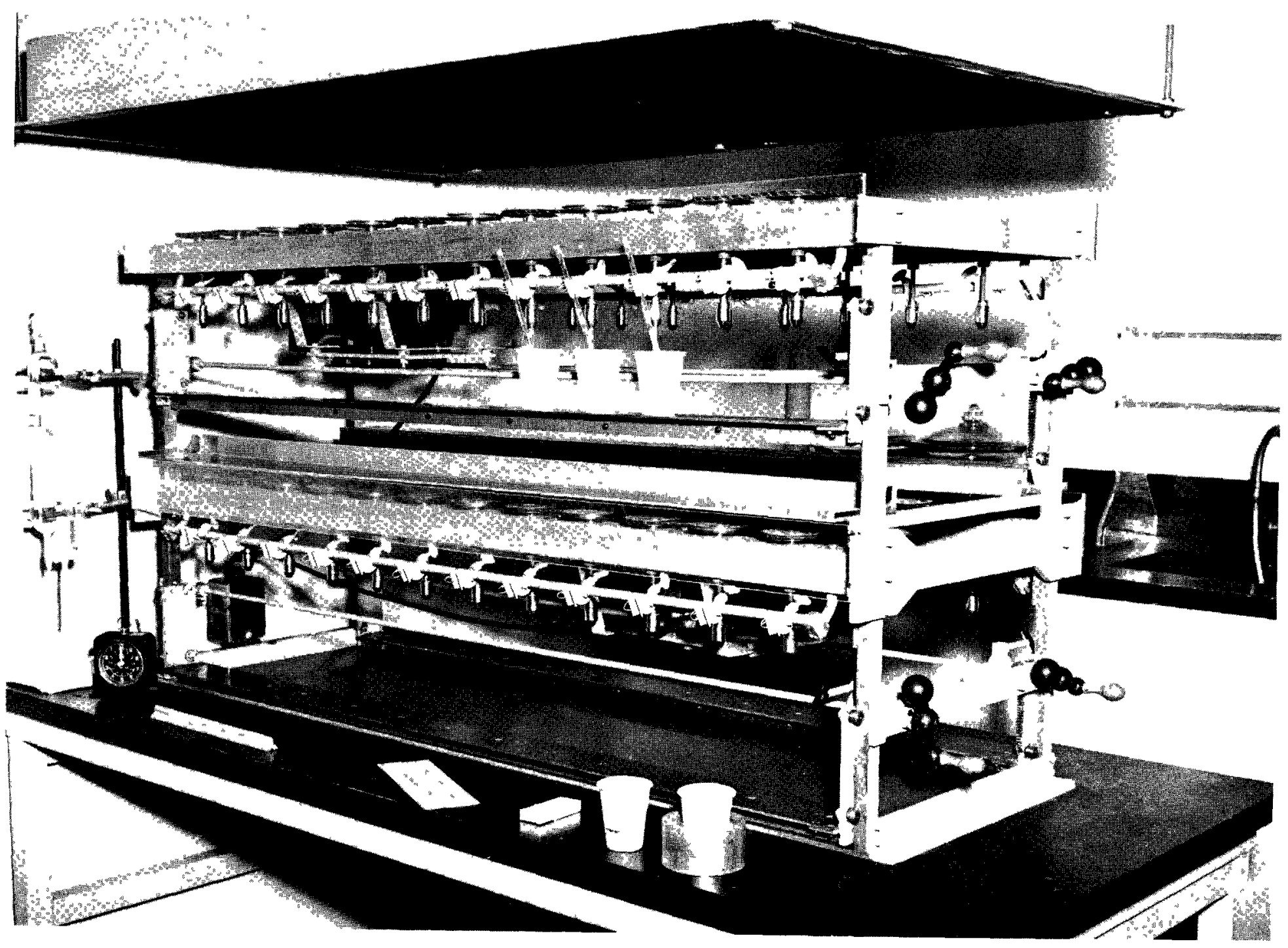

Fig. 1 Equipment used at Mound Laboratory for plating and extraction of polonium from aqueous specimens. 
fixed in the side. The mark is inscribed by giving the cup a quarter turn in the socket of the jig.

The semiautomatic dispenser for the $6 \mathrm{~N}$ hydrochloric acid delivers exactly $10 \mathrm{ml}$ of acid per sample.

The plating discs are suspended on $21-\mathrm{cm}$ glass rods which are drawn into a hook on one end. Spring-type clamps hold the hooked rod and disc at the proper angle in the solution. When a sample is received it is poured into a cup, the hydrochloric acid added, and the cups placed on the frame on each tier. The stirring paddles, $13 \mathrm{~cm}$ long with a $16-\mathrm{mm}$ paddle, and the copper discs are inserted into the cups. The frame is elevated by a rack-and-pinion arrangement with a counter-balanced crankshaft, or crank, to set the correct height precisely for the stirring operation. The disc holders are clamped in position and the stirring rods inserted into a specially designed friction-type chuck with a spring-loaded shoe inside. This is used in place of the ordinary finger-type chuck and has proved a great time-saver. The drawings on this chuck are available. At the end of the plating operation the discs are dried and sent to the counting room in small pill boxes.

Questions and Answers

Question: Are urine specimens collected from personnel in the working area?

Answer: The samples are not actually collected in the working area. We collect them in a clean change room. When people leave the risk area they go first through a hot change room, strip down and use Sanitreads, and then proceed to a cold change room. The samples are submitted in the cold change room.

Question: Have you compared silver or other metals with copper for plating polonium?

Answer: Silver is the only other metal which we have tried. The efficiency was less with silver than with copper. I believe that there are other materials which would give better extraction efficiency, but we have found copper satisfactory.

Question: What is the urine tolerance figure for polonium used at Mound Laboratory?

Answer: We use $0.2 \mu \mathrm{c}--500 \mathrm{~d} / \mathrm{m}$ on a $24-\mathrm{hr}$ sample. Of course, this is based on $8.3 \mathrm{c} / \mathrm{m}$ per $50-\mathrm{ml}$ sample. We do $50-\mathrm{ml}$ spot samples and then normalize to a 24-hr sample assuming a urine volume of $1500 \mathrm{ml}$. 
Question: Have you found any correlation of the polonium yield per spotsample with the sample density or other variable factors of the individual spot samples comprising a 24-hr excretion?

Answer: The people who did this work are no longer with us, and I did not bring their report with me. They did attempt to find a correlating factor in the specific gravity of the spot samples and in the urine excretion rate. However, no correction factor could be found that could be applied to any given spot sample to obtain the average 24- $\mathrm{hr}$ concentration of polonium.

Question: Have you found copper superior to silver or nickel in removing polonium from solution?

Answer: As I said, we found silver less efficient than copper. We have not done work on other metals. At the time our laboratory was satisfied with copper, and we seem to have inherited it.

Question: Would it be advantageous to plate polonium on only one side of the disc in order to reduce the total counting time?

Answer: We have not done this. This appears to have advantages and would reduce the counting time by a factor of 2 .

Question: What were your figures on the effective half-time of polonium in animals and in humans?

Answer: I believe the intravenous studies on rats showed the half-life as 35 days compared to 36.6 in the study made on humans.

Question: What is the daily urine excretion rate of polonium?

Answer: The percentage excreted in the urine was 0.13 per cent per day after the fortieth day.

Procedures for Polonium Analysis

Urine Analysis

\section{Collection}

Routine work with polonium requires that a weekly urine specimen be submitted for analysis. Samples are submitted in half-pint, waxed, Sealrite food containers, at work, at the convenience of the employee, sometime during the morning of the first day of the work week. The sampling period ends at noon. Since the analysis is based on a $50-\mathrm{ml}$ aliquot, a spot sample only 
is required. At the close of the sampling period, the specimen containers are collected and delivered to the urinalysis laboratory for processing.

\section{$\underline{\text { Procedure }}$}

At the time of assay, a 50-ml aliquot of each sample is poured (after thorough shaking), into a pleated wax-paper cup. The urine is brought to $1 \mathrm{~N}$ by the addition of $10 \mathrm{ml}$ of $6 \mathrm{~N}$ hydrochloric acid. A 1-in. diameter copper disc, punched from 16-gauge copper sheet stock, is suspended from a glass hook, which engages a 3/32-in. diameter hole bored near the edge of the disc, and submerged in a vertical position in each sample. The sample is stirred (at 150 to $700 \mathrm{rpm}$ ) for a 2-hr period with a glass stirring paddle. At the end of the plating time, the copper disc is removed from the solution, washed in tap water and air dried. The alpha particles emitted by the attached polonium are counted with a low-background proportional alpha counter. The sum of the counts on the two sides measures the amount of polonium extracted from the specimen.

\section{$\underline{\text { Reagents and Apparatus }}$}

1. Sealrite food containers, waxed, half-pint size

2. $6 \mathrm{~N} \mathrm{HCl}, \mathrm{CP}$

3. Multiple-unit stirring apparatus

4. One-inch-diameter copper discs (16-gauge) with a 3/32-in.diameter hole near the edge of the disc

5. Glass rod with hook to suspend disc in solution

6. Glass paddle to stir solution

7. Pleated waxed paper cups 6 oz (Lily Tulip Company)

8. Low-background proportional alpha counter.

Feces Analysis

Collection

Any employee requested to submit a feces sample is provided a onepint Sealrite food container for the purpose of collecting the 24-hr sample. 


\section{$\underline{\text { Procedure }}$}

Twenty-five to $50 \mathrm{~g}$ of collected sample are placed in an $800-\mathrm{ml}$ Kjeldahl flask together with $100 \mathrm{ml}$ of distilled water and $10 \mathrm{ml}$ of concentrated nitric acid. Heat is applied to the flask and the feces are dissolved in the dilute nitric acid. After the feces are in solution, the sample is cooled to $25^{\circ} \mathrm{C}$. One hundred milliliters of nitric acid are added and the sample is reheated. Cooling the sample before the addition of nitric acid eliminates a violent reaction between the acid and the sample. If foaming occurs in the neck of the flask, the temperature is reduced by immersing In a cold-water bath. Addition of nitric acid continues until a reddish-yellow solution is obtained. At this point, $\mathrm{NO}_{2}$ fumes cease to be evolved indicating the end of the nitric stage.

Perchloric acid $(25 \mathrm{ml})$ is added to the sample and digestion continued until white fumes of perchloric acid appear. If the sample turns brown in the perchloric acid stage, a small volume of nitric acid is added to clear the solution. After the sample is completely digested, the contents of the flask are colorless or slightly greenish-yellow. The latter is due to residual nitric acid.

\section{$\underline{\text { Precautions }}$}

1. Glass beads are used in all digestions.

2. Apply gentle, uniform heat to samples in the beginning of a digestion and after the addition of large quantities of acid.

3. Do not try handling too many samples at one time in the perchloric acid stage.

4. All digestions are started with dilute nitric solution, thereby eliminating excessive foaming and possible loss of sample.

5. Large bubbles appearing at the center of the flask or beaker indicate the end of the nitric acid stage. At the end of the nitric acid stage, the solution is reddish-yellow in color and the volume of sample should be relatively small.

6. Make sure the nitric stage is complete. This eliminates difficulties in the perchloric acid stage.

7. Perchloric acid may be safely added to hot samples.

8. When perchloric acid begins to oxidize and dehydrates the sample, reduce heat by using asbestos pad over wire gauze. The digestion will supply additional heat due to heat of reaction. 
9. If the sample continuously chars or burns, reduce heat and add a small volume of $1: 1$ nitric acid and perchloric acid solution.

After completion of the digestion, distilled water is added to the hot solution to expel any residual nitric acid and the solution heated to dissolve the soluble salts. The clear solution is transferred into a 500-ml Erlenmeyer flask. Three drops of methyl violet indicator are added and the solution is titrated with 75 per cent sodium hydroxide solution to a $\mathrm{pH}$ of 1 (green). Five-tenths normal hydrochloric acid is then added to the $500-\mathrm{ml}$ mark on the Erlenmeyer flask to bring the solution to the desired volume.* Fifty milliliter aliquots of this solution are then plated on 1-in. copper discs as in the urinalysis procedure. ${ }^{3}$

\section{$\underline{\text { Reagents and Apparatus }}$}

1. 800-ml Kjeldahl flask

2. Distilled water

3. $\mathrm{HNO}_{3}, \mathrm{CP}$ concentrated

4. Heating mantle, with wire gauze and asbestos pad

5. Cold-water bath

6. $\mathrm{HClO}_{4}$ (concentrated), 70 per cent

7. Glass beads

8. Methyl violet indicator

9. $\mathrm{NaOH}, 75$ per cent

10. Sealrite food containers, waxed, 1-pint size

11. Reagents and apparatus used in urinalysis procedure

\section{Blood Analysis}

\section{Collection}

If a blood sample is required, the employee is requested to report to the medical department where the plant nurse withdraws $5 \mathrm{ml}$ of blood. The specimen is then sent to the bio-assay laboratory for analysis.

*A large dilution is used to eliminate precipitates in the assay solution. The precipitate is largely composed of sodium and ammonium chlorate. 


\section{$\underline{\text { Procedure }}$}

The blood specimen is oxalated,* and $1 \mathrm{ml}$ of the sample is pipetted into a 250-ml beaker. Ten milliliters of distilled water and $50 \mathrm{ml}$ of a potassium permanganate - sulfuric acld solution (prepared by adding $40 \mathrm{ml}$ of $18 \mathrm{~N}$ sulfuric acid to $10 \mathrm{ml}$ of saturated potassium permanganate solution) are added, and the solution heated in a water bath for $15 \mathrm{~min}$. If the solution has not become clear at the end of this time, small amounts of a saturated sodium sulfite solution are added until the solution clears. Water is then added to bring the volume to $100 \mathrm{ml}$. This solution is processed in the same manner as a spot-urine specimen (see procedure above). This method may not give satisfactory results in all cases because old blood may not be completely digested.

The perchloric acid digestion, which dissolves both new and old blood easily, may be employed if necessary. According to this method, $20 \mathrm{ml}$ of 70 per cent perchloric acid is added to $1 \mathrm{ml}$ of whole blood in a $125-\mathrm{ml}$ Erlenmeyer flask. The system is heated in a wax bath at $205^{\circ} \mathrm{C}$ until the solution becomes colorless. This step requires 10 to $15 \mathrm{~min}$. The solution is cooled and transferred to a $250-\mathrm{ml}$ beaker and, after enough water is added to bring the volume to $100 \mathrm{ml}$, extracted as noted in the first blood analysis procedure. ${ }^{8}$

\section{Reagents and Apparatus}

A.

1. 250-ml beaker

2. 1-ml pipette

3. Distilled water

4. $\mathrm{KMnO}_{4}-\mathrm{H}_{2} \mathrm{SO}_{4}$ solution (prepared by adding $40 \mathrm{ml}$ of $18 \mathrm{~N} \mathrm{H}_{2} \mathrm{SO}_{4}$ to $10 \mathrm{ml}$ of saturated $\mathrm{KMnO}_{4}$ solution)

5. Water bath

6. Saturated $\mathrm{Na}_{2} \mathrm{SO}_{3}$ solution

7. $\mathrm{Na}_{2} \mathrm{C}_{2} \mathrm{O}_{4}$ 7a. $\mathrm{NaCl}$

8. Reagents and apparatus used in urinalysis procedure

*Using 200 lambda of sodium oxalate solution prepared with $1 \mathrm{~g}$ of sodium oxalate dissolved in $20 \mathrm{ml}$ of 0.9 per cent sodium chloride solution. 
B.

1. 1-ml pipette

2. $\mathrm{HClO}_{4}$ (concentrated), .70 per cent

3. 125-ml Erlenmeyer flask

4. Wax bath

5. 250-ml beaker

6. Reagents and apparatus used in urinalysis procedure.

\section{$\underline{\text { References }}$}

1. Silverman, L. B., "Excretion Analysis as a Monitor for Polonium Exposures," Mound Laboratory Report MLM-M-1443, page 1, November 30, 1944 (Classified).

2. Spoerl, E. S., et al., "Urine Assay Procedure at the Mound Laboratory," Mound Laboratory Report MLM-460-Del, April 14, 1950.

3. Cummins, O. J., and Roesch, D., "Wet-Ashing of Biological Materials (Rat)," unpublished Mound Laboratory procedures, July 23, 1954.

4. Report for Blological Research for September 5 to November 27, 1950, Mound Laboratory Report MLM-527-Del, page 19.

5. Steinberg, G., and Baker, M. L., "Low Activity Urine Analysis," Final Report No. 30, Mound Laboratory Report MLM-M-181, page 11, March 21, 1947 (Classified).

6. Silberstein, H. E., et al., "Polonium Distribution and Excretion Experiments with Animals" in Biological Studies with Polonium, Radium, and Plutonium, R. M. Fink, editor, Div. VI, Vol. 3, pp. 40-53, National Nuclear Energy Series (McGraw-Hill Book Company, Inc., New York, 1950).

7. Report for Biological Research for June 18 to September 17, 1951, Mound Laboratory Report MLM-626-Del, page 73 (Classified).

8. Wolfe, B. S., "Physiology of Polonium," Mound Laboratory Report MLM-M-766, March 1, 1946 (Classified).

9. Report for General Research, October 29, 1951 to January 28, 1952 , Mound Laboratory Report MLM-658, page 23.

10. Curtis, M. L., and Paige, M. K., "Evaluation of the Single-Operation Alpha Counter," Mound Laboratory Report MLM-800, December 8, 1952. 


\title{
THE DETERMINATION OF BERYLLIUM IN BIOLOGICAL MATERIALS
}

\author{
J. H. Harley \\ Health and Safety Laboratory \\ U. S. Atomic Energy Commission
}

Since the procedures for the determination of beryllium are given in detail in a summary at the end of this paper, I shall talk first about the general problems of beryllium exposures, difficulties in interpretation of the data, and a bit about methods for the determination of beryllium.

The full explanation of the mechanism of beryllium poisoning is not clear, but it is certain that the material is toxic in very small quantities. It is also evident that the greatest hazard arises from inhalation of airborne material, chiefly the oxide, although beryl and many other compounds show no toxicity. One of the anomalies associated with beryllium is the twotolerance system--one tolerance for people working with beryllium in a plant and one for the area adjacent to the plant. I know of no other nonradioactive material where this is practiced. This two-tolerance system had its origin in our experience in a beryllium-producing plant located at Lorain, Ohio, in the $1940^{\prime} \mathrm{s}$. Approximately 1 per cent of the population of the area surrounding the plant developed chronic berylliosis. Among the workers in the plant the incidence of disease was around 0.5 per cent. They developed acute berylliosis in contrast to the chronlc berylliosis of the surrounding population. Air sampling showed that the beryllium concentration was much higher in the plant than outside. So the accepted levels were set at $0.01 \mu \mathrm{g}$ of beryllium per cubic meter of air in the vicinity of a beryllium plant and at $2 \mu \mathrm{g}$ per cubic meter as the maximum permissible average daily exposure within a beryllium plant.

If you estimate the possible exposure in the plant neighborhood in Lorain, you find something on the order of $0.5 \mu \mathrm{g}$ per cubic meter. If you extrapolate this for five years, the major operating period of the plant, these people should have taken in approximately $10 \mathrm{mg}$ of beryllium. Beryllium excretion rates are not well established, but as an estimate you could say 
that at the end of a year they would have 1 to 2 per cent, or $100 \mu \mathrm{g}$, of beryllium in the body. From the little data available on urinary excretion of beryllium, one would expect these people to excrete approximately $0.05 \mu \mathrm{g}$ of beryllium per day.

This leads to another anomaly associated with beryllium. A patient will have all the symptoms associated with a diagnosis of chronic berylliosis, yet on urinanalysis we are seldom able to find beryllium. It apparently does not follow that a patient with berylliosis will excrete beryllium in measurable quantities. On the other hand, we do have data on two groups of people working in a beryllium plant. Thirty-two people were working with beryllium hydroxide in an area where the exposure was about three and onehalf times the permissible level of $2 \mu \mathrm{g}$ per cubic meter. This means that these people were taking into the body something on the order of $70 \mu \mathrm{g}$ of beryllium per day. Twenty-four-hour specimens on this group gave an average excretion of $1.4 \mu \mathrm{g}$ per day. A second group of 88 people in this plant worked with beryllium fluorides. Their exposure was approximately four and one-half times the permissible level. Urine specimens from this group averaged about $1.1 \mu \mathrm{g}$ per day. There was considerable variation in excretion by individuals in the groups, but all but three people in the fluoride group excreted a measurable quantity of $0.1 \mu \mathrm{g}$ or more per day. These two examples make it apparent that we cannot follow our usual course and establish a normal excretion level for beryllium in an unexposed population unless we use zero as the background level. So we have, on one hand, people who are exposed to high beryllium concentrations and are excreting very small amounts, and on the other hand, people who have chronic berylliosis and excrete no measurable beryllium.

The few cases of tissue analyses which we have done have not added much to our knowledge. The methods of "collection" used by various pathologists is, in part, responsible for this. The 1/2-g samples which we often receive are suitable for sectioning but are insufficient for chemical analysis. Another method is to divide a lung into several equal parts by weight and send it to several laboratories for beryllium analysis. This has resulted in various answers, differing by as much as a factor of 10 . We finally tried an experiment of our own. We took both lungs from a patient with a history of beryllium exposure, divided one into three equal parts, sent it to three laboratories, and got different answers. The other was homogenized in a blender and three portions of the "soup" sent to three laboratories. The results which we received agreed very well. These differences are not surprising when you consider that one would only expect to find something on the order of $0.25 \mu \mathrm{g}$ of beryllium per $100 \mathrm{~g}$ of lung tissue in a case of berylliosis. In addition the beryllium will not be uniformly distributed throughout the tissue. It is apparent that one will have to use a whole lung, or an 
aliquot of homogenized lung, in order to obtain the beryllium concentration in autopsy material.

This points up a third anomaly. In the Lorain neighborhood survey we found fantastically low concentrations of beryllium at distances of onehalf to three-fourths mile from the plant--the area in which the chronic berylliosis was contracted. At this distance you expect dust particles of about $1 \mu$ in size. The $50-\mu$ rocks should have fallen out in the stack area. One-micron particles should be fairly uniformly distributed throughout the area, but we found marked differences in beryllium concentration at different locations at the same radius from the stack source. Again, the lungs of individuals breathing these $1-\mu$ particles should show a uniform distribution throughout the lung, but this is not what we found. The variations found in both the lung specimens and the air samples are real--they are not due to a single particle landing on the air filter or in the lung and giving a detectable concentration or the beginning of a chronic berylliosis.

Another problem in beryllium studies is the lack of agreement of results between one laboratory and another. Differences in analytical methods may contribute to this. The spectrographic procedure used by Cholak at Kettering Institute in Cincinnati is probably as sensitive a method as any. For high concentrations of about $5 \mu \mathrm{g}$ or more colorimetric methods such as the p-nitrobenzeneazoresorcinol method are good. For lower concentrations, down to the spectrographic level, the fluorometric procedures with morin are quite adequate. The basic difficulty is probably due to the nature of the material analyzed. We find the best agreement on water samples and the poorest agreement on urine samples. Lung samples agree pretty well, but $100 \mathrm{~g}$ of lung tissue contains very little inorganic material.

We have given up the spectrographic procedure at the Health and Safety Laboratory and are using morin exclusively. I believe that this is true of most of the installations with the exception of Kettering. Many other reagents have been explored, but no beryllium specific has been found as yet. The basic problem, of course, is that beryllium has no individual chemical properties. We are now using a simple ion-exchange batch process for the initial purification of beryllium. This offers the advantage of simplicity, speed, and reasonable recovery. We are also spiking each spectal sample with a carrier-free radioberyllium at the end of the wet-ashing procedure and using this as our basis for recovery. Most of the beryllium losses occur in the preliminary separation steps so a measure of the recovery is necessary. Of course, the tracer is not in the same form as the beryllium found in the lung, urine, etc., but the ease with which beryllium compounds go into solution makes this unimportant.

For a time it seemed that beryllium exposures would become more or less academic but, as you have read, beryllium-producing facilities are agaln 
being expanded. Thus, the air-control problem is again acute. The problem here is the length of time that it takes to run a number of air samples. Our plants do not work under laboratory conditions, so our air samples contain much insoluble crud, beryl, and other insoluble beryllium materials. These samples are difficult to put into solution, and the analysis involves chemical separation. The operations are time consuming, and we cannot get the results out in time for the data to be used most effectively for plant hygiene.

A great deal of work is being done on methods for obtaining rapid beryllium results. Fitzgerald at the Knolls Atomic Power Laboratory has studied a direct procedure for collection and spectrographic analysis which avoids these chemical problems. The sampling unit collects dust on a 13/16 Whatman No. 40 paper. After sampling, the filter is rolled up and placed in the cavity of a graphite electrode. The paper is charred and excited by a standard d.c. arc. The absolute sensitivity is of the order of $0.0002 \mu \mathrm{g}$ of beryllium. With a flow rate of 10 liters per minute for $15 \mathrm{~min}$, less than 1 per cent of the Industrial MPL may be detected. This method requires only one-quarter of the time needed for an analysis by standard methods. No data on interference are available, but my feeling is that this procedure is limited to operations having low general dust concentrations in the air. However, I belleve that these conditions are fulfilled at General Electric, so the method is sultable for their use.

There have been rumors of a portable monitor developed by the British. The unit is a small direct-reading spectrograph where the air sample is passed between two carbon electrodes. The beryllium concentration is read directly, supposedly to about $2 \mu \mathrm{g}$ per cubic meter. This would amount to a screening procedure or an alarm system. No written information is available on this instrument, and I have not been able to obtain any data on its performance.

Gold, at the Armour Research Foundation, has been working on a system for determining beryllium on filters by alpha bombardment. The reaction involved is $\mathrm{Be}^{9}+\alpha \rightarrow \mathrm{C}^{12}+\mathrm{n}$. The $\mathrm{C}^{12}$ is in an excited state so that the interaction results in a $4.5-\mathrm{Mev}$ gamma as well as a fast neutron. The counting efficiency for neutrons is low so that most of the effort has been devoted to gamma measurement. They have found that there is a good relationship between the amount of beryllium on the paper and the amount of gamma activity. The present system consists of a 5.6-curie Po ${ }^{21 \theta}$ source with a sodium iodide detector. A discriminator system is used to cut out unwanted gamma. Alpha absorption by the filter has been reduced by using millipore filters. The discriminator reduces the gamma background to some extent, but it is still high. Gold has not found other materials likely to be found in plant monitoring which undergo a reaction releasing a high-energy 
gamma ray, but he has encountered some interference with supposedly inert materials. Work done at Mound Laboratory indicates that aluminum and magnesium have similar reactions. The method has the advantage of being rapid and enabling one to get results on plant air samples soon enough for them to have value in a control program.

The following table gives the relative values obtained for several levels of beryllium in millipore filters:

Table 1

\section{GAMMA SENSITIVITY OF BERYLLIUM ANALYZER*}

$\begin{array}{cr}\text { Beryllium, } \mu \mathrm{g} & \mathrm{c} / \mathrm{m} \text { (net) } \\ 0.55 \pm 0.15 & 172 \pm 28 \\ 0.90 \pm 0.2 & 212 \pm 34 \\ 1.1 \text { to } 1.4 & 234 \pm 30 \\ 4.0 \pm 0.6 & 2,220 \pm 50 \\ 16 \text { to } 19 & 4,360 \pm 60 \\ 21 \text { to } 30 & 5,740 \pm 50 \\ 64 \text { to } 80 & 25,800 \pm 120\end{array}$

*5.6-curie $\mathrm{Po}^{210}$ source, 3-in. crystal, 5/32-in. source-to-crystal distance, 2.67-Mev discrimination, and $1737 \pm 20 \mathrm{c} / \mathrm{m}$ background.

In summary, monitoring for hazard evaluation has been largely limited to airborne dusts. The analysis of urine has not been carried out on a large enough scale to obtain any correlation with exposure. By the time analytical methods were adequate, the range of exposure was not available for correlation purposes. We need more beryllium urine data which can be correlated with known exposure levels.

\section{Questions and Answers}

Question: Do you have any estimates of the amount of beryllium carried home by workers in the Lorain neighborhood cases?

Answer: Out of the number of cases of chronic berylliosis known in this incident, to date it is 19 cases out of a population of about 500; only one case was known to have contact with workers in the 
plant. The patient's only contact with beryllium was with her husband's work clothes. We tried to reproduce her washing operations and found that she was exposed to something like 17 $\mu \mathrm{g}$ per cubic meter for a few minutes each week.

\section{Air Dust Sampling}

One of the important tools in the prevention of beryllium poisoning is the monitoring of the concentration of beryllium in the air. The critical concentration for out-plant air may be as low as $0.01 \mu \mathrm{g}$ per cubic meter, ${ }^{1}$ and it is necessary to measure at the level of $0.001 \mu \mathrm{g}$ per cubic meter. Even with a sensitive method the necessity for taking large-volume air samples leads to many difficulties in the analytical process.

The most widely adopted method for spectrographic analysis for microquantities of beryllium is that of Cholak and Hubbard ${ }^{2,3}$. Barnes, Piros, Bryson, and Wiener ${ }^{4}$ have described a method for urine, tissue, and electrostatic separator dust samples. The general methods of Steadman ${ }^{5}$ and Feld$\operatorname{man}^{6}$ have also been applied to the determination of traces of beryllium.

\section{Collection}

Air dust samples run in this laboratory are collected by drawing known volumes of air through paper filters. Depending on the atmosphere sampled, varying types and amounts of impurities are collected with the beryllium. This makes it necessary to use chemical separations to obtain the beryllium in relatively pure solution.

Although the techniques of air sampling are outside the scope of this paper, certain features of the sampling influence the analytical method and must be described.

In-plant samples are collected on small Whatman No. 41 ashless filter paper. The MPL of $2 \mu \mathrm{g}$ per cubic meter average exposure allows breathing zone samples to be taken for a few minutes at 15 to 20 liters per minute. The small paper and low contamination by normal dust make the analysis relatively simple.

Three types of outdoor filters are in common use: the Whatman No. 41 ashless filter paper, the Mine Safety Appliances Company's All-dust paper No. 2133, and Type $S$ impregnated filter. The first filter, in a $10-\mathrm{cm}$ (4-in.) diameter, allows a sampling rate about 0.7 cubic meter per minute but clogs rapidly in dusty atmospheres. The second paper allows a sampling rate of 1.0 to 1.3 cubic meters per minute but does not clog so rapidly. The Type $S$ fllter, because of the large area of the pleated structure, allows a sampling 
rate of about 2 cubic meters per minute and is very resistant to clogging. However, it has an ash content of over 1 per cent (125 mg per filter), which adds to the impurities in the sample. Blanks run on these papers show up to $0.3 \mu \mathrm{g}$ of beryllium per filter.

\section{Urine Sampling}

Collection

Twenty-four-hour urine specimens or one-liter specimens are collected in polyethylene bottles. These are acidifled with about 1 per cent hydrochloric acid to maintain the beryllium in solution. Because of the low output a large sample is used.

\section{$\underline{\text { References }}$}

1. Eisenbud, Merril, Wanta, R. C., Dustan, Cyril, Steadman, L. T., Harris, W. B., and Wolf, B. S., "Non-Occupational Berylliosis," J. Ind. Hyg. Toxicol., 31, 282-294 (1949).

2. Cholak, Jacob and Hubbard, D. M., "Spectrographic Determination of Beryllium in Biological Material and in Air," Anal. Chem., 20, 73-76 (1948).

3. Cholak, Jacob and Hubbard, D. M., "Spectrochemical Determination of Beryllium," Anal. Chem., 20, 970-972 (1948).

4. Barnes, E. C., Piros, W. E., Bryson, T. C., and Wiener, G. W., Spectrochemical Determination of Beryllium in Microquantitles," Anal. Chem., 21, 1281-1283 (1949).

5. Steadman, L. T., "The Spectrochemical Determination of Beryllium," U. S. Atomic Energy Commission Report AECD-1957, May 12, 1948.

6. Feldman, Cyrus, "Direct Spectrochemical Analysis of Solutions," Anal. Chem., 21, 1041-1045 (1949).

\section{Bibliography}

Klemperer, F. W. and Martin, A. P., "Determination of Traces of Beryllium in Biological Material," Anal. Chem., 22, 828-831 (1950).

Laitinen, H. A. and Kivalo, Pekko, "Fluorometric Determination of Traces of Beryllium," Anal. Chem., 24, 1467-1471 (1952). 
Sax, N. I. and Kramlich, W., "A Fluorophotometric Determination for Beryllium in Microquantities," General Electric Data Folder No. DF 505L100.

Sterner, J. H. and Eisenbud, Merril, "Epidemiology of Beryllium Intoxication," A. M. A. Arch. Ind. Hyg. and Occupational Med., 4, 123-151 (1951).

Summary of Beryllium Methods Used at HASL

The following outlined procedures show the actual operating procedures used at HASL for determination of beryllium in various materials. The determinations are given, then the separation procedures for various matrices.

This material is a condensation of unpublished material and the following previously published articles from the Health and Safety Laboratory:

"Fluorimetric Determination of Trace Amounts of Beryllium," by G. A. Welford and J. H. Harley, Am. Ind. Hyg. Assoc. Quart., 13, 232-234, 1952;

"Spectrographic Microdetermination of Beryllium in Air Dust Samples," by G. E. Peterson, G. A. Welford, and J. H. Harley, Anal. Chem., 22, 1197-1200 (1950).

Spectrographic Procedure

Spectrograph, Baird 3-meter grating, 50- $\mu$ slit.

Source Unit, Baird with Sola constant-voltage transformer.

Lower Electrode, National Carbon Co., 0.25-in. (0.6-cm) nominal

diameter graphite, regular grade, $6.3 \times 50 \mathrm{~mm}$, with a $4.5-\mathrm{mm}$ cup, $5 \mathrm{~mm}$ deep.

Upper Electrode, Same type, sharpened to a $45^{\circ}$ point.

Excitation, Direct current arc, $10 \mathrm{amp}, 2 \mathrm{~min}$.

Densitometer, Baird non-recording.

The aluminum-beryllium precipitate obtained on collection of beryllium after preliminary separation is dissolved and made up to $1 \mathrm{ml}$ with $1: 7 \mathrm{sul-}$ phuric acid.

The cupped electrodes are weatherproofed with a solution of Duco cement in acetone and filled with sodium chloride to within $1 \mathrm{~mm}$ of the top by tamping in a dish of the salt. The weight of sodium chloride has been found to be $45 \pm 5 \mathrm{mg}$ in all cases measured. Three electrodes for each sample are treated with $0.05 \mathrm{ml}$ of the solution described above and dried in an oven at $110^{\circ} \mathrm{C}$. 
The lower electrode is made the anode in the arc. The electrode spacing of $4.5 \mathrm{~mm}$ and the length of anode extending from the water-cooled electrode holders are set manually, using an auxiliary lens and screen. The arc image is focused on the grating with a long-focal-length quartz lens.

The spectrum is recorded on Eastman Type 33 plates, and standard development and fixing procedures are used. The densities of $\mathrm{Be}$ at 2348.6A, $\mathrm{Be}$ at $2650.8 \mathrm{~A}$, and $\mathrm{Al}$ at $2367.1 \mathrm{~A}$ are read, and the ratio of the suitable beryllium line to the aluminum line is used as the analytical function.

No plate calibration procedure is used; the ratio $\mathrm{Be} 2348 \mathrm{~A}: \mathrm{Al}$ 2367A gives a straight line from 0.005 to above $0.2 \mu \mathrm{g}$ of beryllium on the electrode, as does the ratio $\mathrm{Be} 2650 \mathrm{~A}: \mathrm{Al} 2367 \mathrm{~A}$ from 0.1 to $10 \mu \mathrm{g}$ of beryllium. Standards are run periodically, and the analytical curve is remade if excessive shifts appear.

The sample burns smoothly during the excitation period, giving a negligible background on the plate. Although reburning the electrodes shows that some beryllium is left, complete burning does not increase sensitivity or accuracy because an increased continuous background is produced.

Fluorometric Procedure

After preliminary separation, the beryllium is collected using $2.5 \mathrm{mg}$ of aluminum as a carrier. This precipitate is dissolved and made up to $5 \mathrm{ml}$ with $2 \mathrm{~N}$ sodium hydroxide. Triplicate $1-\mathrm{ml}$ aliquots are treated with $1 \mathrm{ml}$ of 0.0001 per cent morin and diluted to $5 \mathrm{ml}$ and the fluorescence is measured.

The fluorometer was designed and built by the Instruments Branch of HASL. It has a sensitivity of $0.005 \mu \mathrm{g}$ and is linear over a range of 200 .

A General Electric AH-4 100-watt lamp is the source of ultraviolet, and a photomultiplier tube, amplifier, and microammeter are used in the measuring circuit. Four solution cells of $5 \mathrm{ml}$ capacity are held in a slide, the ultraviolet entering at the side and the fluorescence being measured at the top. This allows blank and triplicate samples to be measured in rapid succession.

A 0.01 per cent quinine sulfate solution is used to set the high end of the scale, varying the phototube voltage until the quinine solution reads 20,000 scale units. The total scale range is 100,000 units in four decades. The blank reading is about 20 units, and $0.01 \mu \mathrm{g}$ of beryllium gives an average reading of about 20 units above the blank. 
Purity and Concentration of Morin

A 1 per cent acetone solution of technical grade morin as purchased from Eastman Kodak was originally used. Further dilutions were made in absolute ethyl alcohol. This morin in a sodium hydroxide concentration of $0.2 \mathrm{~N}$ per tube gave very high blanks which ranged from 26 to 34 on the thousand scale. These values, on the lower end of the least sensitive scale, are undesirable. Further dilutions did not increase the ratio of beryllium to blank to a workable difference.

A method of purification of morin modified from that of J. F. Bonner, University of Rochester Report UR-111, has been used successfully. A 1 per cent solution of morin in acetone is used as the basis for further dilutions with distilled water. This solution is stable for months if kept in the dark. This reagent gives a reagent blank of 40 deflection units at a 0.0001 per cent concentration.

Morin of better than 99 per cent purity is avallable from L. Light \& Co., Ltd., Colnbrook, Bucks, England, at reasonable prices. This morin has been used in our laboratory for the past two years and gives a reagent blank of 20 deflection units at 0.0001 per cent concentration. A typical calibration curve is linear over a range of 200. Addition of $0.005 \mu \mathrm{g}$ of beryllium gives 10 deflection units above the blank.

\section{Reagents}

1. Standard beryllium solution - Spectroscopically pure beryllium sulfate $(0.982 \mathrm{~g})$ is dissolved in $100 \mathrm{ml}$ of 20 per cent hydrochloric acid and diluted to 1 liter with water. The nominal composition is $50 \mu \mathrm{g} / \mathrm{ml}$. Further dilutions are made with water just before use.

2. Standard aluminum solution - A solution containing approximately $2.5 \mathrm{mg} \mathrm{Al} / \mathrm{ml}$ is prepared by dissolving aluminum nitrate nonahydrate in water. The solution is prepared in large amounts and standardized gravimetrically.

3. Oxine solution - $12 \mathrm{~g}$ of oxine (8-quinolinol, 8-hydroxyquinoline) is dissolved in glacial acetic acid and made up to $100 \mathrm{ml}$. The solution should be prepared fresh weekly and kept out of the light when not in use.

4. Versene, or tetrasodium salt of ethylenediaminetetraacetic acid.

5. Resin IR 4B (OH) 
6. 2N sodium hydroxide

7. Quinine sulfate solution - 0.01 per cent solution of quinine sulfate in 1 per cent sulfuric acid

8. 1 per cent morin in acetone solution - $1 \mathrm{~g}$ of purified morin dissolved in $100 \mathrm{ml}$ of acetone, store in refrigerator

9. 0.0001 per cent morin solution - made up by diluting the 1 per cent morin solution with distilled water, store in refrigerator

\section{Chemical Procedure for Beryllium in Type S Filter Discs} acid.

1. Place the filter paper in a 400-ml beaker and add $100 \mathrm{ml}$ of nitric

2. Stir the paper to a pulp with a stirring rod, and add $5 \mathrm{ml}$ of sulfuric acid and $10 \mathrm{ml}$ of perchloric acid.

3. Cover beaker, place on hot plate, and evaporate to sulfur trioxide fumes.

4. Evaporate with additional nitric acid till no further reduction in color results.

5. Transfer the solution to a $50-\mathrm{ml}$ platinum dish, add $2 \mathrm{ml}$ of hydrofluoric acid, and evaporate to dryness on a sand bath. tube.

6. Add $4 \mathrm{ml}$ of hydrochloric acid and transfer to a 50-ml centrifuge

7. Adjust the volume to $20 \mathrm{ml}$ with distilled water.

8. Neutralize with ammonium hydroxide to the first iron or aluminum hydroxide precipitate.

9. Add $2 \mathrm{ml}$ of glacial acetic acid. Add hydrochloric acid dropwise until precipitate is dissolved.

10. Add $5 \mathrm{ml}$ of 12 per cent oxine solution.

11. Adjust the $\mathrm{pH}$ to 6 with ammonium hydroxide; using Hydrion test paper as an indicator.

12. Filter the sample through No. 41 Whatman paper into a clean 125-ml separatory funnel.

13. Wash the precipitate with water.

14. Combine the washings with the original filtrate. 
15. Extract filtrate and washings with $10 \mathrm{ml}$ of chloroform to remove excess oxine.

16. Repeat Step 14 until chloroform layer is clear.

17. Transfer aqueous phase to a clean $50-\mathrm{ml}$ centrifuge tube and adjust $\mathrm{pH}$ to 8 .

18. If precipitate appears at this point, collect precipitate and repeat Steps 6 through 17.

19. If the solution is clear, add $1.0 \mathrm{ml}$ of aluminum nitrate solution.

20. Centrifuge and discard supernatant liquid.

21. Dissolve precipitate with $5 \mathrm{ml}$ of $2 \mathrm{~N}$ sodium hydroxide.

22. Transfer to $15-\mathrm{ml}$ centrifuge tube, adjust volume to $10 \mathrm{ml}$ with distilled water.

23. Add $3 \mathrm{ml}$ of disttlled water to three fluorometric cells.

24. Pipette $1 \mathrm{ml}$ of sample solution into each cell.

25. Add $1 \mathrm{ml}$ of morin solution (0.0001 per cent) to each cell and stir solution thoroughly.

26. Place cells in fluorometer, standardized with 0.01 per cent quinine sulfate solution, and record galvanometer deflections.

27. Prepare reagent blanks in manner outlined in Steps 23 through 25.

\section{Chemical Procedure for Beryllium in Tissue}

1. Dry sample in oven at $110^{\circ} \mathrm{C}$ overnight.

2. Ash aliquot of sample until all organic matter is destroyed.

3. Follow procedure outlined for Beryllium in Urine from Step 2 through Step 16.

4. Follow procedure outlined for Beryllium in Type S Filter Discs at Step 19 through to completion.

Chemical Procedure for Beryllium in Water

1. Add a $250-\mathrm{ml}$ aliquot of the sample to a $400-\mathrm{ml}$ beaker. 
2. Add $50 \mathrm{ml}$ of nitric and $5 \mathrm{ml}$ of sulfuric acids and evaporate to trioxide fumes.

3. Follow procedure for Beryllium in Type S Filter Discs from Step 5 through Step 27.

\section{Chemical Procedure for Beryllium in Urine}

1. Place sample in a beaker with an equal volume of nitric acid and evaporate until all organic material is destroyed.

2. Pick up residue with distilled water and adjust volume to $100 \mathrm{ml}$.

3. Add $1 \mathrm{ml}$ of 10 per cent Versene solution.

4. Add 60 per cent sodium hydroxide until $\mathrm{pH}$ is 11.5 to 12 .

5. Add a quantity of wet resin (approximately $5 \mathrm{~g}$ dry weight of IR-4B (OH) and stir mechanically for $1 \mathrm{hr}$.

6. Add $100 \mathrm{ml}$ of Versene wash solution and stir for $10 \mathrm{~min}$. liquid.

7. Filter through No. 41 Whatman paper and discard supernatant

8. Punch a hole into filter paper and wash resin quantitatively with $6 \mathrm{~N}$ hydrochloric acid into a 250-ml beaker.

9. Stir solution mechanically for $1 / 2 \mathrm{hr}$.

10. Filter through No. 41 Whatman paper, collect the filtrate, and discard the resin.

11. Add $75 \mathrm{ml}$ of nitric acid and $5 \mathrm{ml}$ of sulfuric actd and evaporate samples to dryness.

12. Repeat ashing step until all organic matter is destroyed.

13. Dissolve the residue in approximately $4 \mathrm{ml}$ of hydrochloric acid and transfer with distilled water into a 50-nil centrifuge tube.

14. Adjust volume to $20 \mathrm{ml}$.

15. Neutralize with ammonium hydroxide.*

\footnotetext{
*If precipitate appears at this point, separation of iron and aluminum is incomplete, and the ion-exchange procedure must be repeated.
} 
$\underline{\text { Resin Preparation for Beryllium in Urine Procedure }}$

1. Wash $500 \mathrm{~g}$ of $\mathrm{IR}-4 \mathrm{~B}(\mathrm{OH})$ resin with 8 liters of water (20-min wash interval). Decant solution.

2. Make up in 8 liters of 10 per cent diammonium citrate solution (wash for $20 \mathrm{~min}$ ).

3. Repeat Step 2.

4. Wash in water, 4-liter portions, three times (20 min each washing).

5. Add 8 liters of 1 per cent hydrochloric acid $(20 \mathrm{~min})$.

6. Repeat Step 5 for $10 \mathrm{~min}$.

7. Make up 10 per cent hydrochloric acid solution and wash for $20 \mathrm{~min}$.

8. Repeat wash of 10 per cent hydrochloric acid for $10 \mathrm{~min}$.

9. Wash with 4 liters of water for $20 \mathrm{~min}$.

10. Wash again with 4 liters of water for $10 \mathrm{~min}$.

Procedure for Beryllium in Soil

1. Weigh out $50 \mathrm{ng}$ soil sample to the nearest $0.1 \mathrm{~g}$.

2. Add $500 \mathrm{ml}$ of $6 \mathrm{~N}$ hydrochloric acid and stir mechanically for $30 \mathrm{~min}$.

3. Add $200 \mathrm{ml}$ of water, filter, and wash precipitate with $100-\mathrm{ml}$ portions of $6 \mathrm{~N}$ hydrochloric acid.

4. Evaporate, leach to approximately $30 \mathrm{ml}$, and transfer to $100-\mathrm{ml}$ platinum dish.

5. Add $2 \mathrm{ml}$ of concentrated sulfuric acid and approximately $20 \mathrm{ml}$ of hydrofluoric acid.

6. Pick up residue with $2 \mathrm{ml}$ of concentrated hydrochloric acid and transfer to 50-ml centrifuge tube.

7. Add $10 \mathrm{ml}$ of 6 per cent oxine solution.

8. Add $2 \mathrm{ml}$ of acetic acid.

9. Bring $\mathrm{pH}$ to 6 with ammonium hydroxide, filter into 125-ml separatory funnel, and extract oxinates with three 10-ml portions of chloroform. 
10. Discard chloroform extractions, transfer the water solution to a 50-ml centrifuge tube, and boil off the chloroform.

11. Bring $\mathrm{pH}$ to 8 with ammonium hydroxide and add approximately $1 \mathrm{ml}$ of aluminum solution. Centrifuge and discard the supernatant.

12. The solution is now ready for fluorometric analysis.

13. Follow procedure for Beryllium in Type $S$ Filter Discs from Step 21.

Bibliography

DiGiovanni, H. J., Graveson, R. T., Dwork, B., "Photofluorimeters for Determination of Uranium and Beryllium Concentrations," Health and Safety Laboratory Report NYO-4508, August 18, 1954.

Peterson, G. E., Welford, G. A., and Harley, J. H., "Spectrographic Microdetermination of Beryllium in Air Dust Samples," Anal Chem., 22, 1197-1200 (1950).

Welford, G. A., Peterson, G. E., Fast, I., and Harley, J. H., "Determination of Beryllium in Urine" (Unpublished).

Welford, G. A., and Harley, J. H., "Fluorimetric Determination of Trace Amounts of Beryllium," Am. Ind. Hyg. Assoc. Quart., 13, 232-234 (1952).

Welford, G. A., et al, "Isolation of Beryllium in Urine," Health and Safety Laboratory Report, 1952. 


\title{
APPLICATION OF STATISTICAL METHODS TO BIO-ASSAY
}

\author{
J. W. Healy \\ Hanford Atomic Products Operation \\ Richland, Washington
}

\section{Introduction}

The word statistics is used with two common meanings. The first is as a replacement for the word "data." Thus, any compilation of information concerning a given subject can be referred to as the "statistics" of that subject. In the meaning of interest in this paper, it refers to the principles and methods which have been developed for handling these data, that is, the collection, presentation, analysis, and interpretation of numerical data.

In a more limited sense we can refer to statistics as the formal manipulation of information which will aid in the interpretation of the meaning of a given set of data. It is important to recognize at the start that statistical methods are not a primary aim but are rather the application of techniques which permit one to obtain a scientific estimate of the meaning of the information. The final numbers from a statistical examination are useless until they are used in an interpretation.

This paper is intended to describe some of the simpler statistical techniques which may be used in a bio-assay program and to indicate the usefulness of some of these concepts in bio-assay work.

\section{Statistical Methods}

Before discussing the application of statistics to bio-assay, we will review some of the more elementary concepts of statistics. These concepts, while simple, provide the basis for a large fraction of the statistical methods which are applicable for general use. We will avoid the more advanced and detailed treatments which require a serious study of statistics. 
The Frequency Distribution

The important fundamental in all statistical studies is the realization that measurements of any nature are not absolute. Thus, a given measurement repeated a large number of times will produce results which are not completely reproducible but show deviations from one extreme to another. These variations occur widely in work with radioactive materials, where measurements are concerned with the results of the disintegration of individual atoms, each of which occurs strictly according to the laws of chance. In such a situation it is possible to measure the counting rate of a sample ten times and arrive at ten different answers. All of these answers are correct for the particular time period involved, but none of them necessarily represents a true value of the average number of atoms which disintegrate in a given time. In such a situation it is impossible to describe an absolute value of the disintegration rate. We can only obtain an estimate of this rate from the measurements.

An array of data obtained by measurements over a number of time intervals will indicate that there is a most probable value which occurs more times than any other. If one moves in either direction from this most probable value, the number of times which the value in question is obtained diminishes. This information can be arranged as a frequency distribution which expresses the probability of obtaining a given value for a particular measurement. Thus, in dealing with statistical techniques, we do not simply compare two numbers, each of which is mathematically accurate, but rather we compare the frequency distribution curves, each of which has no definite boundary. This results in a situation where conclusions must be made according to the probability with which a given event may occur.

In the ideal situation we know the frequency distribution curve of the entire group of numbers with which we are working. Thus, using a simple analogy, if we are concerned with the frequency distribution of the height of sixth-grade students in the city of New York, it is possible to measure the height of every individual in the sixth grade and therefore draw a distribution curve for the entire group. If in a later experiment one does not have the facilities to measure the height of every individual, then a sample of 5 or 10 per cent of the children may be measured and the distribution curve of the entire group used for comparison. In this analogy the frequency distribution curve obtained from every individual in the sixth grade is known as the frequency distribution of the "population," while the curve obtained from the sample is an estimate of this frequency distribution as obtained from the sample. This concept is of considerable importance in interpreting results since, in most cases, the distribution of results in the entire population is not known and can be estimated only from the frequency distribution of the sample. 
There are several types of frequency distribution or probability distribution which can be expressed by relatively simple mathematical equations. The most fundamental is the binomial law which states that the probability of an event occurring $r$ times in $n$ independent trials is

$$
P_{r}=\frac{n !}{r !(n-r) !} p^{r} q^{n-r}
$$

In this expression $p$ is the probability of the event occurring in a single trial, and $q$ is the probability of the event not occurring.

A simple approximation of this type of distribution for many problems is given by the Poisson Law,

$$
P_{\mathbf{r}}=\frac{(n p)^{r} e^{-n p}}{r !}
$$

The Poisson distribution gives a good approximation to the binomial when $r$, the number of times the event occurs, is small compared with $n$, the number of trials; and $p$ therefore represents the probability of a rare event in a single trial. Since np represents the expected number of successes, Eq. 2 can be written as

$$
\mathbf{P}_{\mathbf{r}}=\frac{\mathbf{M}^{\mathbf{r}} \mathrm{e}^{-\mathrm{M}}}{\mathbf{r} !}
$$

In Eq. $3 \mathrm{P}_{r}$ is the probability of having the event, $r$, occur; and $M$, which represents $\mathrm{np}$, is the most probable value of $r$. This distribution is skewed to the right, that is, the curve is not symmetrical about the center but has a tail in the higher probability region (Fig. 1). The distribution of individual measurements on radioactive sources, where the total number of events measured is small as in most bio-assay work, will follow the Poisson equation if the equipment is working properly and no other factors are involved. Note that the degree of skewness in this distribution is dependent upon the magnitude of $r$, the average number of events measured. The wide spread at low values of $r$ is of considerable importance in bio-assay since many of the measurements are in this range. Note also that these curves are drawn with flat tops. The Poisson distribution as it is written is a discrete, not a continuous, distribution.

For large values of $M$, that is measurement of large numbers of individual events, the Poisson distribution approximates the more familiar normal or Gaussian distribution curve. This occurs when more than about 30 


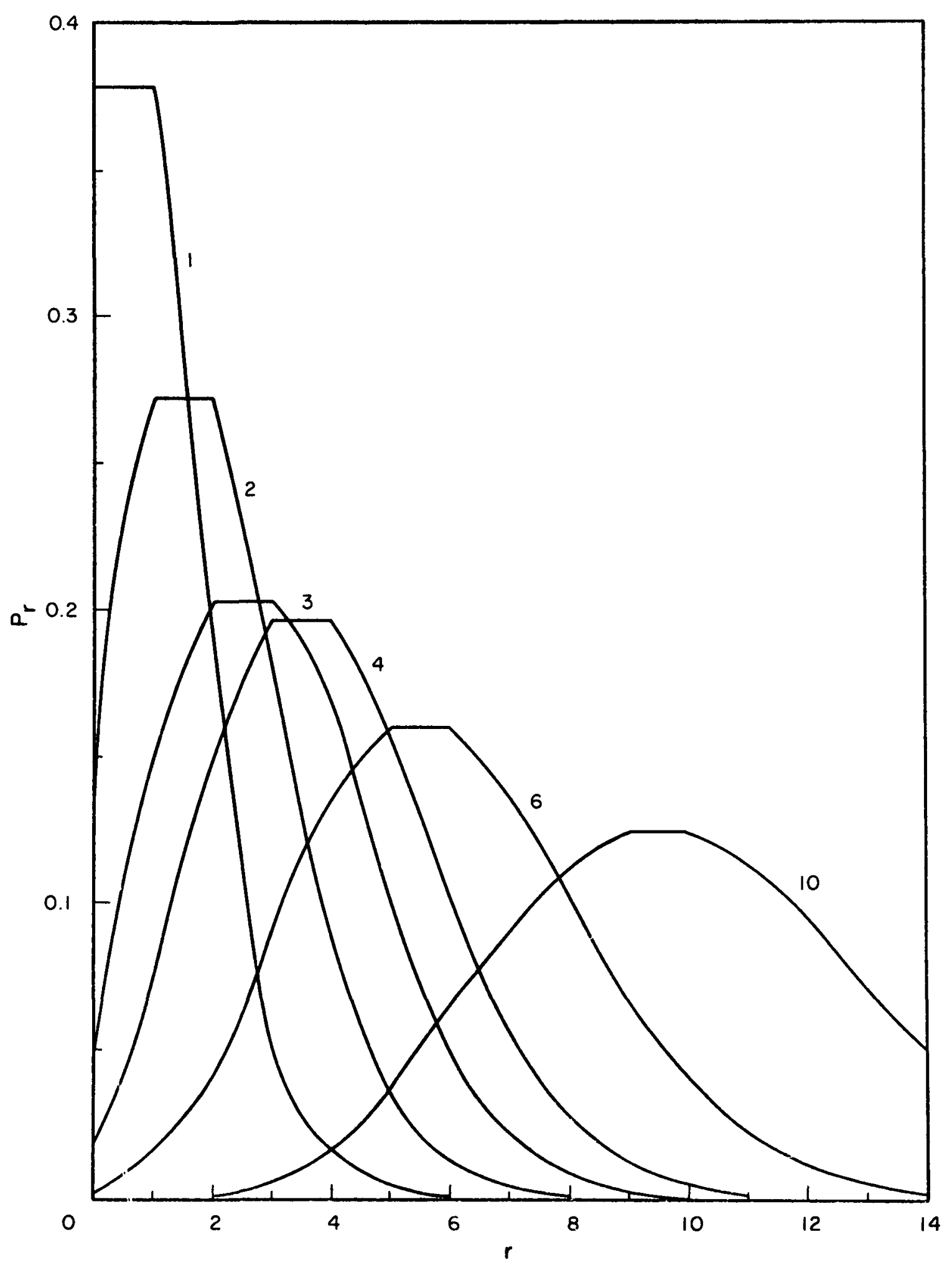

Fig. 1 Poisson distribution.

$$
=40-
$$




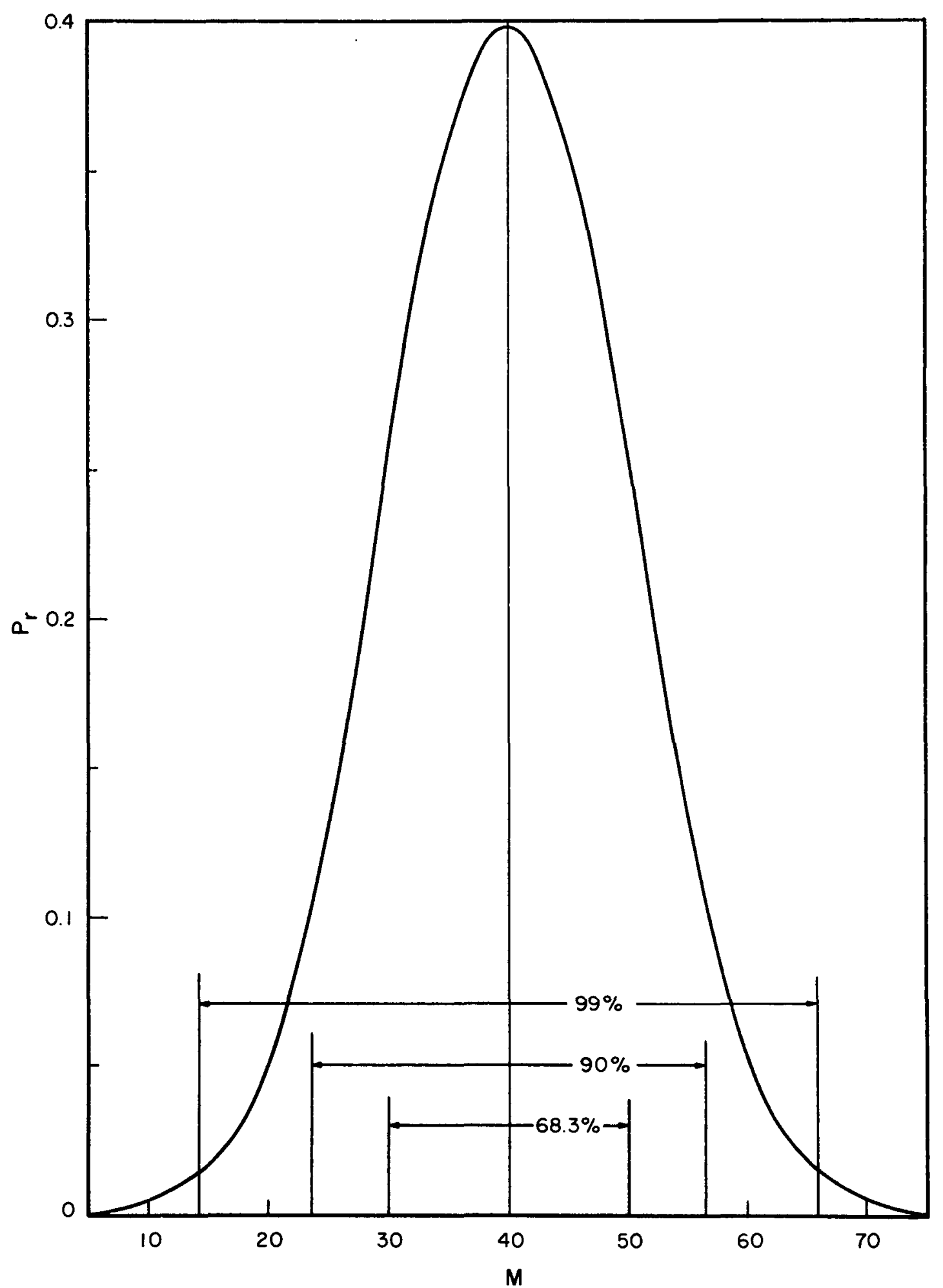

Fig. 2 Normal distribution curve. 
events are measured. The normal curve is symmetrical on both sides of the mean with the mean, corresponding to the value observed most frequently (Fig. 2). The width of a normal distribution curve which describes the spread of data is adjustable so that it may be fitted to data regardless of the error in the measurement. The normal distribution curve is given by Eq. 4,

$$
f=\frac{n}{\sigma \sqrt{2 \pi}} e^{-(r-M)^{2} / 2 \sigma^{2}}
$$

In this equation $f$ is the frequency of occurrence of any value of $r, n$ is the total number of observations made, and $\sigma$ is the standard deviation or the distance from the mean to the point of inflection of the curve. The constants of the curve in units of the standard deviation are listed in mathematical tables so that the curve can be used without further calculations.

\section{Statistical Measures}

In the use of statistical concepts certain measures are applied to provide the criteria for interpretation. The best known of these criteria is the mean, or average, value. In statistics the mean is considered as the arithmetic average of all the values measured. In terms of the normal frequency distribution one-half of the area under the curve falls on either side of the mean. The median is defined as the value which has equal numbers of items on either side. Note that the median can differ from the mean since the median is not weighted for the distance by which the individual value differs from the mean. The mode is the value at the point around which the items are most heavily grouped or, in other words, the most frequent item to appear. It should be noted that these three items are not equivalent. Only in a normal distribution, which is symmetrical about the center, will these items be the same number.

Although these measures give an indication of the central density of the midpoint of the distribution, some additional numbers are needed to provide a measure of the spread of the data. The most familiar and most widely used of these measures is the standard deviation which is defined by Eq. 5,

$$
\sigma=\sqrt{\frac{\Sigma(\mathrm{x}-\overline{\mathrm{x}})^{2}}{\mathrm{n}}}
$$

Where $\mathrm{x}$ represents the individual values, $\bar{x}$ represents the mean, and $n$ 
represents the number of values. In this equation the sum of the square of the individual deviations is used since an algebraic summation of the deviations will result in zero. Alternate methods of describing the deviation indicate the range covered by the individual points or, in some cases, the average deviation without regard to arithmetical sign. These measures suffer from the inability to define quantitatively the width of the probability distribution which is encountered. Although Eq. 5 describes the standard deviation in the simplest possible terms, it is not convenient for use on the calculator. Equation 6 may be derived from Eq. 5 and permits independent summation of the data without the necessity of first calculating an average.

$$
\sigma=\sqrt{\frac{\Sigma \mathrm{x}^{2}-\mathrm{x} \Sigma \overline{\mathrm{x}}}{\mathrm{n}}}
$$

Thus, running tables of summations of individual values squared and of individual values may be made and the standard deviation calculated at any time. If it is later desired to add additional data, it is sufficient to obtain the summations for the new data, combine with the old data, and recalculate the standard deviation. This equation is useful in cases where continuing control on a process is required.

In measurement of the disintegration rate of a radioactive material, the standard deviation may be estimated from the square root of the total number of events measured. Thus, the accuracy of measurement of a radioactive material is proportional to the square root of the total number of disintegrations actually registered in the measuring device.

The standard deviation, physically, is a measure of the width of the normal distribution curve. It represents the distance from the mean within which 68.3 per cent of all of the measurements taken in a similar manner will fall. Thus, in the tables of the normal distribution curve, when the value of the abscissa is 1 , the area between the positive and negative abscissa will be 0.683 . The probability of obtaining a value different from the mean is then a function of the ratio of the measured deviation from the mean to the standard deviation. For our purposes the standard deviation can be considered as applying only to the normal distribution curve although it has definite meaning in the mathematics of the other curves.

If a series of measurements, such as of the height of a group of school children, are made, the standard deviation resulting indicates the probability of variation of the individuals. If the entire group of measurements is repeated several times, the variations in the means should be less than the variations in the individuals. An estimate of the variations expected in the means of such measurements is given by 


$$
\sigma_{m}=\frac{\sigma_{i}}{\sqrt{n}},
$$

where $\mathrm{n}$ is the number of individuals in the group.

\section{Statistical Tests}

In the application of statistics to any set of data the interpretations are made in terms of probability. Thus, an event may be highly probable as shown by the statistical tests, and we then consider that the data show the trend that we are investigating. It is never possible in statistics to state conclusively that a given interpretation should be made.

In testing data by the statistical method, we assume that individual variations are strictly random and due to chance. We then test the probability of finding variations as large as those observed and from the results of this test conclude whether the hypothesis of chance variations is valid. In many of the tests we assume that all of the data are drawn from the same population and that there are no significant differences, and then proceed to test the truth of this hypothesis. The point at which we should draw a line as to a significant difference is frequently arbitrary and usually depends upon the purpose of the analysis. Thus, if we are testing the uniformity of gradation on a ruler to be sold at the 5- and 10-cent store, a probability of difference of perhaps 5 to 10 per cent may be acceptable. If however we are testing the gradation on a precision slide rule, then the probability criteria should probably be set at $1 / 100$ or $1 / 1000$.

The simplest statistical test, and one which is used frequently, is the testing of an individual against a series of data to determine whether there is a significant difference. This test is known as the "t test" since the values of the abscissa in the plots of the normal distribution curve are usually labeled as the $t$ values. This test is given in simple form by Eq. 8,

$$
t=\frac{x-\bar{x}}{\sigma_{p}}
$$

The value of $t$ is simply the ratio of the individual deviation from the mean to the standard deviation. This gives the value of the abscissa on the normal distribution from which the area of the normal curve between this value and the mean, or the probability of occurrence, can be read directly.

If two sample means are to be tested, that is, if the significance of the difference between two series of samples is desired, the problem becomes somewhat more complex since the standard deviation of the difference between 
the two means must be computed. The equation for such a $t$ test is

$$
t=\frac{\bar{x}_{1}-\bar{x}_{2}}{\sqrt{\frac{n_{1} \sigma_{1}^{2}+n_{2} \sigma_{2}^{2}}{n_{1}+n_{2}-2}\left(\frac{1}{n_{1}}+\frac{1}{n_{2}}\right)}} .
$$

If more than about 32 samples are involved in this test, the $t$ distribution approximates the normal distribution, and the values can be read from the tables which can be found in the Handbook of Chemistry and Physics or most texts on statistics. If a smaller number of readings are involved, the limits must be obtained from statistical tables which list the probabilities for the given number of readings.

In many cases it is desirable to know whether the frequency distribution involved in a particular series of samples is different from the frequency distribution which is assumed in making the tests. As an example, in a large number of samples it may be desired to test the actual fit of data to a Poisson or normal distribution. This is carried out by means of the $\chi^{2}$ test,

$$
\chi^{2}=\sum \frac{\left(f_{c}-f\right)^{2}}{f_{c}}=\frac{n \sigma^{2}}{\sigma_{p}^{2}}
$$

The values of $\chi^{2}$ for various probabilities may be obtained from standard statistical tables.

In the application of statistics it is frequently desired to know the trends which may occur between two variables and to indicate whether these apparent trends can be caused strictly by chance or whether the probability of such chance can be estimated. This is carried out by correlation techniques. A correlation coefficient which describes the probability of a linear relationship appearing simply due to chance variations in both variables is given by Eq. 11,

$$
r=\frac{\Sigma x y-\bar{x} \Sigma y}{\sqrt{\left(\Sigma x^{2}-\bar{x} \Sigma x\right)\left(\Sigma y^{2}-\bar{y} \Sigma y\right)}} .
$$

The correlation coefficient can never exceed 1. It does not express the probability itself, but this probability may again be obtained from tables. 
If a significant correlation is indicated, the linear-regression line or estimating equation may be obtained by using the least-squares fit. This technique minimizes the sum of the squares of the deviations of the individual measured values from the estimated line. The expressions for the leastsquares estimate of the parameters $a$ and $b$ of the linear function given in Eq. 12 are found in Eqs. 13 and 14:

$$
\begin{aligned}
& \mathrm{y}=\mathrm{a}+\mathrm{bx}, \\
& \mathrm{b}=\frac{\Sigma \mathrm{xy}-\overline{\mathrm{y}} \Sigma \mathrm{x}}{\Sigma \mathrm{x}^{2}-\overline{\mathrm{x}} \Sigma \mathrm{x}}, \\
& \mathrm{a}=\overline{\mathrm{y}}-\overline{\mathrm{x}} \mathrm{b} .
\end{aligned}
$$

\section{$\underline{\text { Process Needs }}$}

\section{Excretion Relations}

One of the fundamental needs in any bio-assay program is the relationship between body content of a radioisotope and excretion rate. Such relations can be obtained only by experimental data with the associated uncertainties in measurements. Since the elimination rate of the radioisotope has a trend with time, it is necessary to establish a curve which describes the excretion rate at various times after administration of the radioisotope. In order to obtain the best curve possible, such relationships are derived from the experimental data by the methods of least squares. The most familiar equations of this type are those for the excretion of plutonium, which were derived by Langham. Note that in this case a power function of the time was used to describe the excretion rate. As a result the correlation was carried out with the logarithm of the fractional excretion and the logarithm of the time. This technique places the data in the form of a linear equation so that the linear correlation methods previously described may be used.

In this particular case the data on quantity administered was readily available so that the excretion rates could be expressed in terms of the percentage of the administered quantity. In most problems involving accidental exposure to other isotopes the quantities administered are not known, but valuable information on the rate of excretion of these isotopes can be obtained by similar correlations using the absolute quantities excreted per day. Figure 3 illustrates a correlation between the quantity of strontium excreted per day by a worker who was exposed to radiostrontium and the time. 


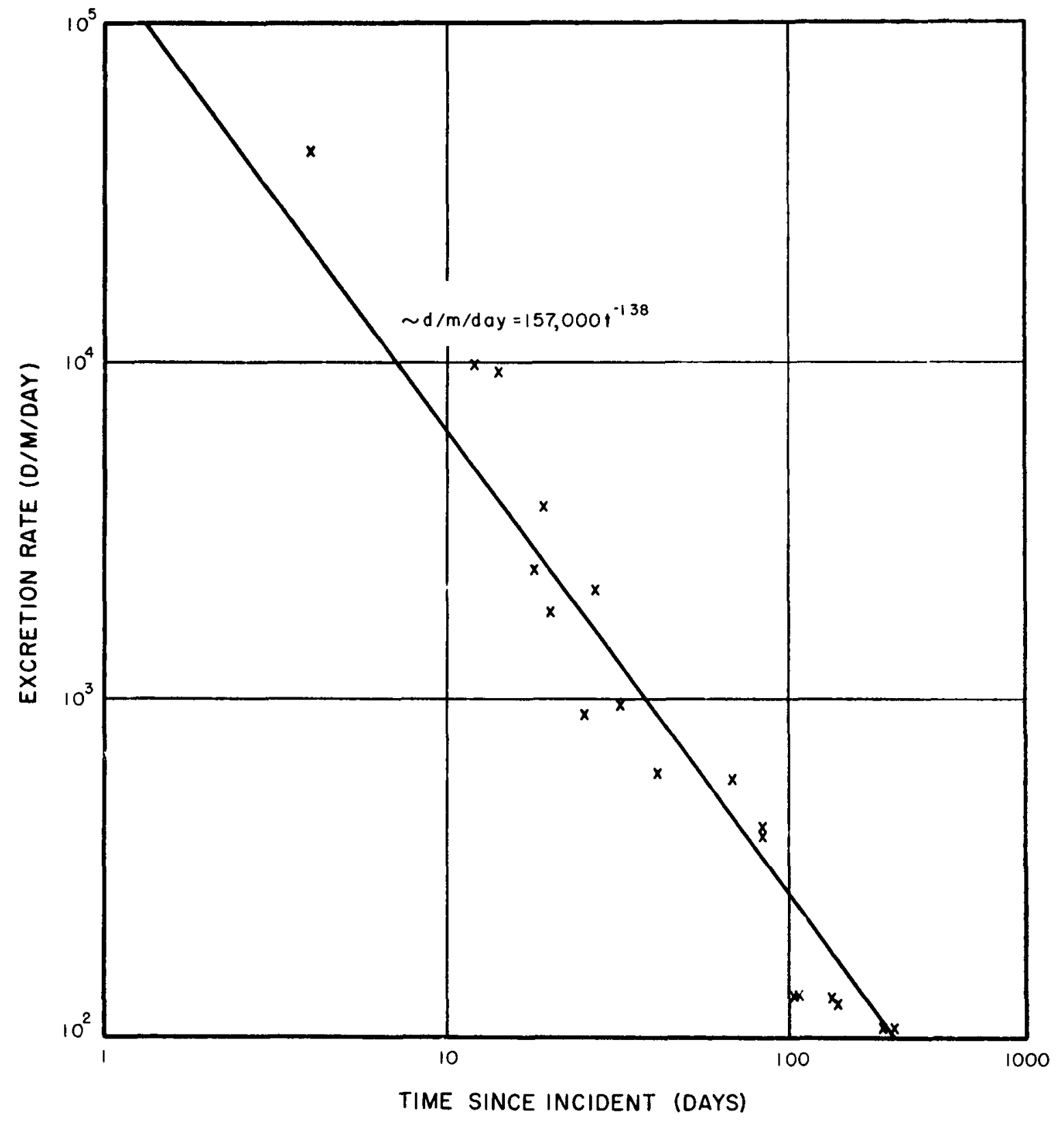

Fig. $3 \mathrm{Sr}^{90}$ excretion. 
Similar correlations are frequently of value in the interpretation of biological data and later extrapolation to humans. Figure 4 illustrates the excretion of ruthenium administered to the rat by several different techniques.

\section{Process Control}

Statistical techniques for the control of the various analytical procedures used to obtain the measurement of the excreted quantity are as applicable to bio-assay work as to any other process. The main differences in technique arise from the need to estimate relatively small quantities of material and these differences result in conclusions on individual samples drawn from the measurement of a relatively small number of events.

\section{Control Charts}

A control chart is a graphical method for obtaining a $t$ test on the significance of the difference between an individual value and a previously established normal performance. It is used to indicate the probability of deviations of individual samples from this performance and to draw attention to trends which will cause eventual difficulty.

Two types of control charts may be defined depending upon the definition of "normal" performance. In many cases the limits are set by comparison to an expected performance which will not change over a long period of time. In this case the limits may be set and not changed over the period of the chart. Such a chart could be prepared for the performance of a counter that is expected to produce results which are dependent only upon the number of particles entering the active volume and not upon the particular settings involved. Even in this case, however, a major change in circuitry (such as the replacement of a GM tube) may result in a change in the mean response to the radiation which will require a new control chart although the limits are still controlled by the theoretical deviation.

Figure 5 illustrates this type of control chart for a counter. This control chart was calculated for a counter in which the individual measurements are made for a $10-\mathrm{min}$ period at this counting rate. The standard deviation is given by the square root of the total number of counts. The 90 and 99 per cent limits are obtained by multiplying the standard deviation by the ratio of the area of the normal distribution curve to the 90 and 99 per cent limits to the standard deviation. In this case this turns out to be 1.645 times the standard deviation for the 90 per cent limits and 2.575 times the standard deviation for the 99 per cent limits. Normally there will be a distribution of points about the mean indicated by the solid line. Approximately 


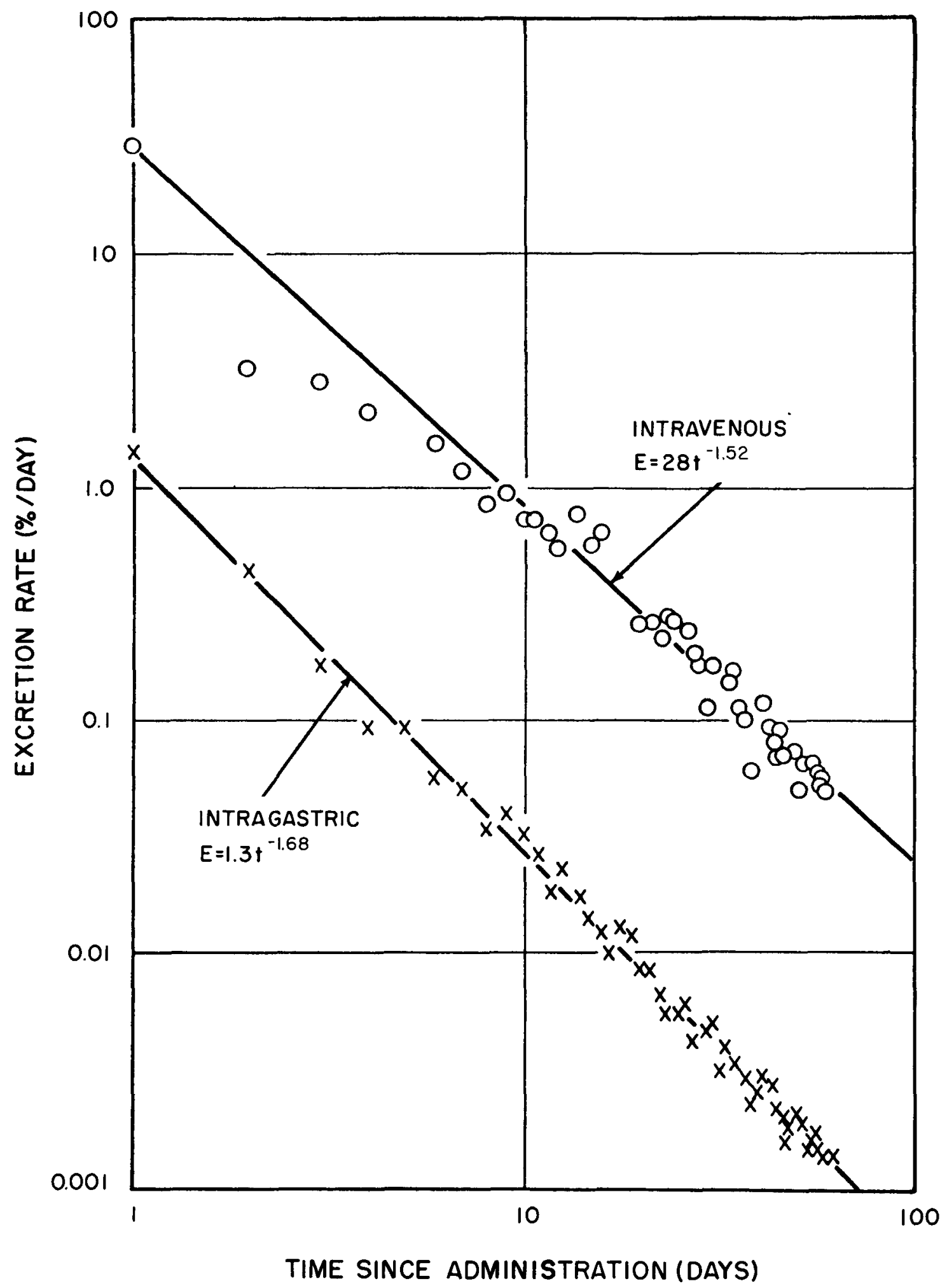

Fig. 4 Urinary excretion of ruthenium in the rat. 


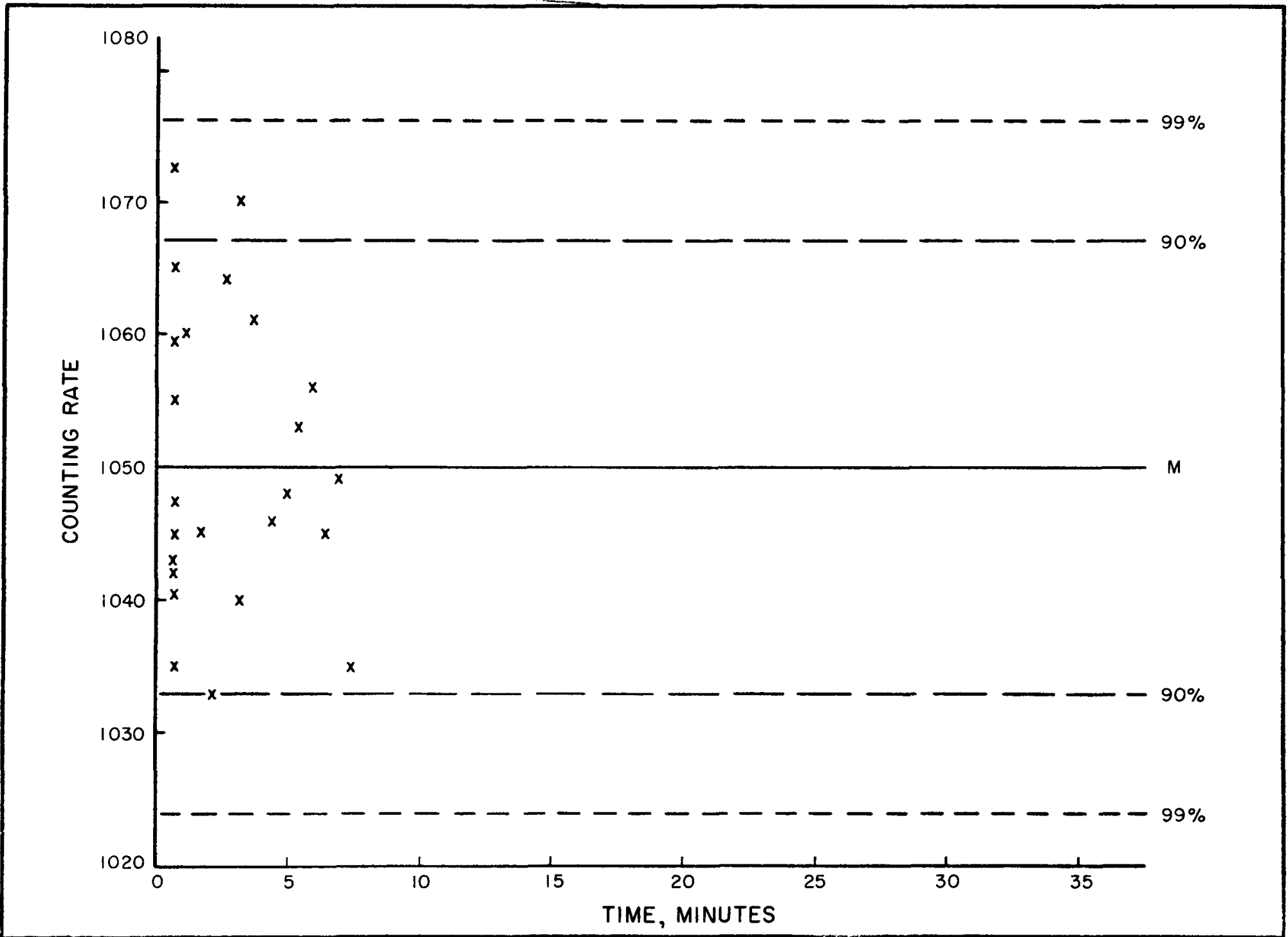

Fig. 5 Control chart for a counter. 
one point in ten falls outside the 90 per cent limits and one point in 100 outside the 99 per cent limits. This type of chart simply shows the deviation of the individual points from the mean compared to the deviation which is expected.

In other procedures the interest is in the performance over a relatively short time so that the limits could change. For example, in the establishment of chemical yields to be used in correcting analytical results, it is desired to obtain a value representative of the present practices and to control the procedures according to the present practices (Fig. 6). In this case the limits are frequently obtained by using the data for the past period and dropping the obsolete information each time the more recent data are available. Thus, the mean is representative of the present performance, and data for the next period will be compared against the most recent limits.

The establishment of limits on the control chart depends upon the type of frequency distribution encountered. For a normal distribution the standard deviation represents the range within which 68.3 per cent of the values may be obtained. The limits for other probabilities may be obtained by considerations of the area of the distribution curve represented by other ordinates. Thus, two standard deviations represent 95.5 per cent of the area while three standard deviations represent 99.7 per cent. In practice the 90 and 99 per cent limits occur at 1.645 and 2.575 standard deviations from the mean. For other distributions, such as the Poisson, the limits are nonsymmetrical and must be obtained from tables. In some cases the results follow a frequency distribution which is described by functions other than the normal, Poisson, or binomial. In this case the limits may be obtained by fitting to a more complicated expression or in the extreme case by plotting the distribution and estimating from this unique plot.

In the use of such a chart it should be remembered that a proper set of data describes a frequency distribution about the mean. It is therefore as troublesome to see all of the points fall within the 90 per cent limits as it is to see most of them fall outside.

\section{Counter Controls}

A counter designed to measure individual particles is ideally suited for statistical controls since the ideal performance provides a well-defined frequency pattern. If all particles entering produce a pulse with no spurious pulses added by the counter, the results will be described by the Poisson distribution; or if the counting rate is high enough, the results will be described by a normal distribution with the standard deviation given by the square root of the total number of events measured in the given period. 


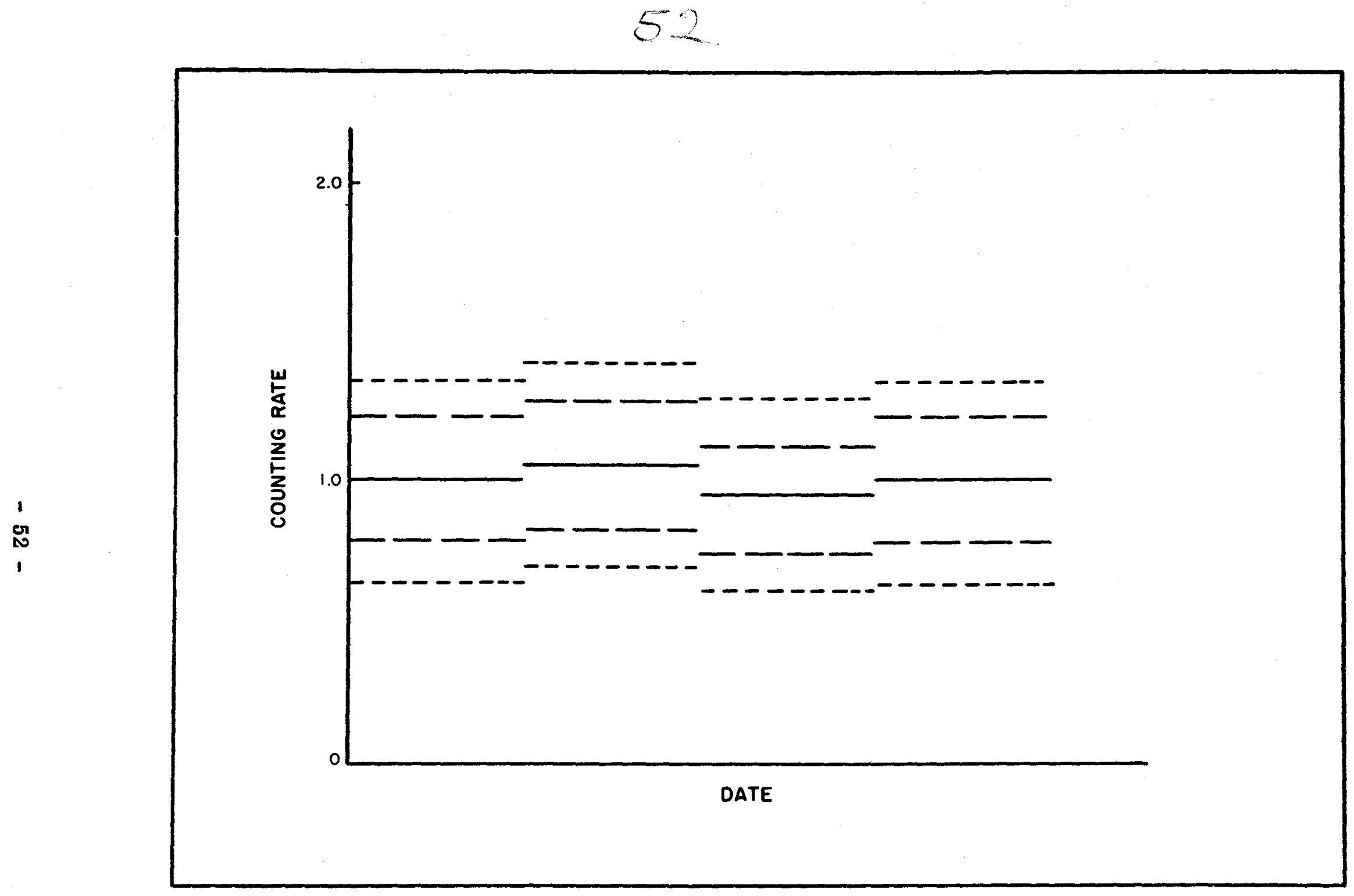

Fig. 6 Control chart of chemical yields. 
Thus, once the mean is established, it is possible to immediately obtain the limits which should be applied to a proper counting device.

The usual counter requires control of two items which enter into the calculation of the quantity of radioisotope in the sample: (1) background and (2) the response to a standard quantity of radiation. The background control provides information on the trends which may occur due to contamination or faulty circuitry; however it is usually not valuable to indicate minor defects because the counting rates are low and the limits correspondingly broad. The standard response is of importance in assessing the counter geometry to be applied and in keeping measurements internally consistent.

A control chart is usually kept for each counter with measurements of the control sample at least once per day. The interpretation of such data must include the probability of occurrence. Thus, one point outside of the 90 per cent limits has little meaning although a recheck may be desired. One point outside of the 99 per cent limits is less probable and a recheck should be mandatory. Two points in succession outside of the 99 per cent limits should require some counter checkup, or if the situation persists, a re-establishment of the correction factors. In addition a long-range review of the performance may be made by noting the number of points outside of the 90 and 99 per cent limits. Although these numbers will not be exactly 10 or 1 per cent since there will be fluctuations in this fraction, a major deviation can indicate trouble. If a small number of points fall outside the given limits, it may indicate that there is uniform pulsing in the electronic circuitry associated with the counter.

In establishing such a control chart, a series of measurements on the standard source will be required to establish the mean value of the counting rate. This mean will have an uncertainty which is less than the uncertainty of the individual value by the square root of the number of measurements. It is possible that this estimate of the mean may be different from the true mean so that points will fall off the control chart. As additional measurements are made, they may be added to the initial values, thereby decreasing the uncertainty of the mean and minimizing this problem.

As an interesting corollary to the chance nature of the disintegrations, it may be noted that the probability of time intervals between disintegrations (therefore between individual counts) is an exponential function of the form $e^{-\bar{x} t}$. This states that the most probable time of obtaining a second event is immediately following another event. In cases where the individual events are displayed as a "pip" on a strip chart, it is possible to examine the statistical behaviour of the counter during a single measurement by measuring the time intervals between events and comparing to the exponential distribution by a chi-square test. This may be simplified by a series of scales for various counting rates in which the distances within which given fractions of 
the events should fall are marked. The scale can then be moved along the chart and the fraction falling within the 10 per cent limits, 20 per cent limits, etc., measured directly.

\section{Spiked Samples}

In most analytical laboratories a series of samples to which a known amount of material is added is analyzed along with the actual samples. These data not only provide a basis for later correction of the samples but also indicate the performance in the laboratory on a continuous basis.

The application of control techniques here requires prior information on the laboratory performance since the sources of error are random and the standard deviation is not a theoretical function of other relations. In practice this control may be kept on the most recent data, such as for the last three or six months. This is best accomplished by dropping the earlier month and adding the most recent month in the calculation of the mean and the standard deviation. Such a control accounts for improved procedures and ensures that the yield is current.

The application of such a control permits estimation of the variability introduced into the final answer from this source as well as the reliability of any individual sample. The use of this knowledge depends upon other operating practices. For instance, in a laboratory where a spiked sample is analyzed with each batch of samples, it may be desirable to throw out any data obtained in a batch where the yield falls outside of the 99 per cent limit, or a separate assessment of the effect of low yield may be made on these samples. Although this appears drastic, if it is assumed that the spiked sample is representative of the entire group, then a probable indication of defective performance exists. Longer term trends in the performance will be indicated by a high percentage of values falling outside of the limits on one side or the other, although such trends will be minimized by using current data for the estimation of limits.

An alternate use of this type of control is in the study of the performance of individual analysts. In this case different symbols or colors appear on the chart with the same criteria used to indicate individual differences from the mean.

Data Interpretation

Proper interpretation of data involving the random fluctuations encountered in low-level measurements of radioactive material requires a knowledge of the fundamentals of statistics. In many cases this results in a less 
definitive answer than is desired or which is written down by "intuitive" reasoning. One of the advantages of statistical methods, however, is that they assist in defining the limitations which can be placed on any interpretation.

\section{Positive Samples}

In assessing the results of a bio-assay program, some criterion to differentiate positive samples from the large body of data is required. This can be accomplished by the use of a technique similar to that of a test, where the deviation required to indicate a given probability is used as the criterion. Thus, a sample which differs from the mean by 1.645 standard deviations has a 90 per cent probability of differing from the body of data from a cause other than chance, while a difference of 2.575 standard deviations indicates a 99 per cent probability. It should be noted here that statistical methods make no attempt to ascribe a cause to this difference. They simply point out that a difference exists and that the cause should be investigated.

The data which serve as the basis of comparison require some attention. Again, many groups analyze a "blank" sample, which consists of urine obtained from people known to have no exposure, along with each group of samples. This procedure serves as a control on the possibility of crosscontamination in the laboratory or carrying of extraneous radioactive materials from the reagents. It also provides an excellent source of data on the results of the analytical procedure on a noncontaminated population. These data may also be used as a basis for comparison of a group with exposure potential against a zero potential population. Such comparisons may lead to indications of routine exposure of the entire group since the sensitivity available from a series of measurements is always greater than from individuals.

If the bulk of the samples is essentially background, then the test for significance may use the over-all data from sample analyses. Here the assumption is made that the effect of an occasional positive sample is negligible and that a sample which deviates significantly from the mean is drawn from another population. In this case the positive sample should be eliminated from the tabulation and the average and standard deviation recalculated.

\section{Detection Limits}

One of the early lessons from a study of statistics is that the number 0 has no meaning when applied to a measurement. That is, we cannot state that there is no radioactive material present in a sample since the errors 
in measurement always permit the possibility of its presence. As an example, suppose that in the nuclear track technique for plutonium we measure a given area of the film and find no tracks. According to the Poisson distribution we could find no tracks 37 per cent of the time if there were a true average of one track in this area. There would be a 1 per cent chance of obtaining zero tracks if the true number were 4.6 , or a 10 per cent chance if the true number were 2.3. Thus, a reading of 0 indicates a 99 per cent probability that there are not more than 4.6 tracks. Increasing the area read to ten or 100 times will not change the situation since the initial area was not specified in the discussion; however, increasing the area will decrease the sensitivity per unit area.

Such a measurement, then, on a low sample results in the conclusion that there is less than a given amount of radioactive material in the sample. Any result above this may be considered to be positive.

In contrast to this "positive value" we can define a "detection limit" as the quantity of radioactive material which, if present in the sample, will give a positive signal the desired fraction of the time. For illustration we will use the 99 per cent probability level. The positive value defined earlier is one which is different from the background of the process (the blank samples). If samples containing radioactive materials equivalent to exactly this value are analyzed, the results will describe a frequency distribution about the positive value as a mean. This infers that 50 per cent of the samples will be less than positive and will not be detected.

Thus, at this limit the probability of detection will be 50 per cent. As the quantity of radioactive material is increased, the probability of detection is increased. In order to provide a 99 per cent probability of detection, the quantity of radioactive material must equal the mean of the distribution which has a lower 99 per cent limit at the positive value, i.e., the upper 99 per cent limit of the blank samples (Fig. 7). This detection limit, then, indicates the quantity of radioactive material which will result in a positive sample 99 times out of 100 .

In calculation of the detection limit, the estimation of the frequency distribution of the positive samples may be made from spiked samples by comparing to theoretical distributions. This will point out variables in the procedures which may affect the results and will permit compensation. The same distribution function is then applied to obtain the detection limit. Extrapolation of this function to a new mean is simple if it obeys one of the theoretical distributions such as the Poisson. Routine data on samples with varied quantities of radioactive materials will assist in extrapolation of other functions but require additional control samples. In most cases an assumption of the same percentage deviation at all values of the average will provide a reasonable approximation. 


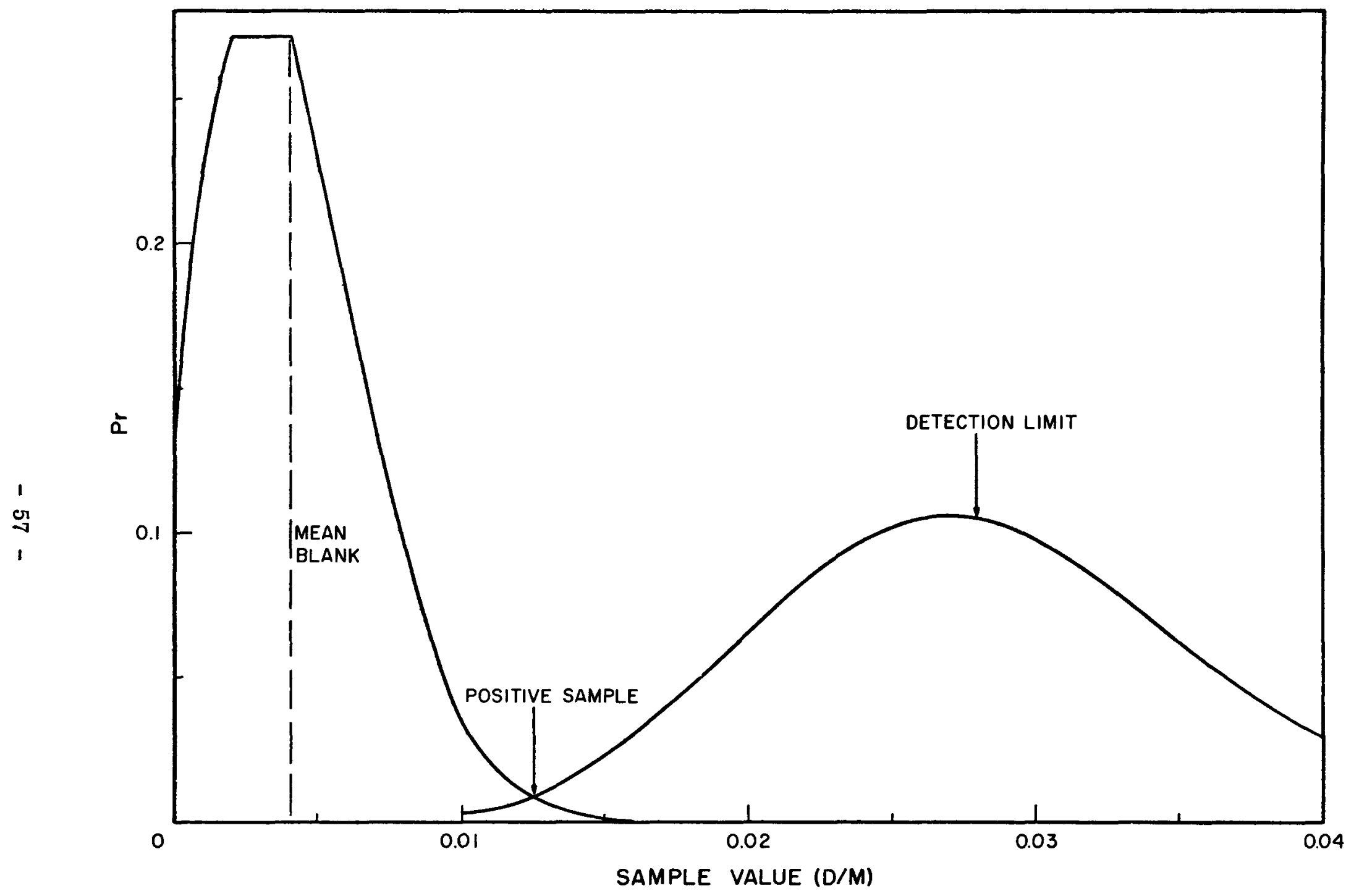

Fig. 7 Detection limit. 


\section{Resamples}

The statistical manipulations described provide a criterion on which decisions can be made; they do not make the decision. The probability limits and application of the criteria are left to the judgment of the individual. In this fashion the question of re-examination of positive values is left to the operator. One criterion would be to resample and reprocess all samples which have greater than 99 per cent probability of being positive. This automatically requires an increase of 1 per cent in the workload, even if no positive samples are obtained, and results in confirmation of a truly positive result at this limit in only a fraction of the cases. It does, however, provide a rigid criterion of checking which may detect possible longrange trends. It is also the only criterion to be used if the detection limit is to be defined as given above since the detection limit is keyed to this quantity.

Other criteria are possible, however, depending upon the economics and the need for sensitivity. For example, in a very inexpensive process where the detection limit is close to the permissible limit, a criterion of 95 or even 90 per cent may be applied for resampling. In any process it may be desirable to remeasure samples above the 90 or 95 per cent limit although the resample limit remains at 99 . Such a technique is generally inexpensive and provides a method of concentrating measurement time on those samples which are most uncertain. Consistent application of such a policy will improve the detection limit by decreasing the deviation.

\section{Averages}

The estimate of the body burden from bio-assay results may be accomplished from individual values, although the usual practice is to obtain a number of samples to provide increased accuracy. Statistical methods may be used to estimate the uncertainty of the final answer by taking into account the deviation of the individual values. The problem is complicated by the trend of the excretion function with time; but if this function is known, individual values may be corrected before averaging. This may be illustrated by the hypothetical example of plutonium excretion following a single administration as given in Table 1 . These calculations indicate that the average excretion rate at one day is $10 \mathrm{~d} / \mathrm{m}$, with a 68 per cent probability that additional samples will give between 8.1 and $11.9 \mathrm{~d} / \mathrm{m}$. 
Table 1

\section{HYPOTHETICAL PLUTONIUM EXCRETION \\ $E=0.002 t^{-0.74}$}

Time since
Administration,
days

2
10
17
30
140
320
476
723

Excretion Rate, $\mathrm{d} / \mathrm{m} /$ day

5.6

1.7

1.0

0.95

0.35

0.10

0.10

0.08
Excretion Rate

Corrected to 1 Day, $\mathrm{d} / \mathrm{m} / \mathrm{day}$

10.2

9.4

8.1

11.7

13.6

7.1

9.6

10.5

If the experiment were repeated a number of times, the means should show a standard deviation of $1.9 / \sqrt{10}$, or $0.60 \mathrm{~d} / \mathrm{m}$. It may then be stated that there is a 68 per cent probability that the mean is within $0.60 \mathrm{~d} / \mathrm{m}$ of the true mean. In extrapolation to the body burden these values of the uncertainty provide an index to the possibility of exposure. Note that in the above example, no weighting has been made for the accuracy of the individual measurement. Obviously the value of $5.6 \mathrm{~d} / \mathrm{m}$ can be measured more accurately than the $0.08 \mathrm{~d} / \mathrm{m}$. Thus, the values at the beginning of the table could be given additional weight in the estimation of the average and deviation.

\section{Conclusions}

In summary, the usual bio-assay procedures are ideally suited for the application of statistical techniques. Many valuable conclusions may be drawn from the application of even elementary concepts. This paper has emphasized some of the aspects which can be applied by an individual with some knowledge of statistical methods and has purposely omitted some of the finer details which could be obtained from a statistician's treatments. 


\section{Questions and Answers}

Question: How do you report your results?

Answer: We take into account the plating area, the time of exposure, etc., and then express our results in so many $\mathrm{d} / \mathrm{m}$ with the Poisson limits allowed.

Question: With a frequency distribution which does not have the same limits of error on either side of the mean, how do you express the precision?

Answer: You're worried about the skewness of the Poisson distribution compared to a normal distribution. We report, for example, $4.6_{-2}^{+3}$.

Question: Does the calculated standard deviation actually fit the data obtained by measurement?

Answer: Since I am of a skeptical nature, I have plotted the data for some counters and found that the standard deviation is the square root of the total count, etc. I found excellent agreement between the calculated and the measured deviations. I have not done this, however, for low background counters. 


\section{LOW-LEVEL ALPHA COUNTING AND TECHNIQUES OF PULSE-HEIGHT ANALYSIS}

Richard J. Watts

Los Alamos Scientific Laboratory

University of California

Los Alamos, New Mexico

The purpose of my paper is to present some of the practical problems we have encountered in low-level alpha counting and then to turn attention to pulse-height analysis and discuss up-to-date developments in this fleld.

Low-Level Alpha Counting

We have been interested in low-level alpha counting for bio-assay work since 1945. At that time we received some of the original Simpson Alpha Counters built by Hallicrafters, from the Metallurgical Laboratory, and we have been using them ever since.

These counters, of course, have been rebuilt many times and other types have been added so that I can summarize the situation by saying that at present we have 12 counters in operation. Our average counting times are on the order of $2 \frac{1}{2} \mathrm{hr}$, so that if we are pressed we can do about 36 samples per day.

In low-level alpha counting, backgrounds are all-important. For many years we were doing this work in the basement of a temporary building. Because of an unfortunate accident, we found that it was very difficult to get our backgrounds below $9.0 \mathrm{c} / \mathrm{hr}$ with 50 per cent of a $4 \pi$ geometry.

In 1953 we moved to a special counting room in the new Health Research Laboratory and we found that with the same instruments our background dropped to about $6 \mathrm{c} / \mathrm{hr}$.

Two years ago we were able to drop our backgrounds to $3.6 \mathrm{c} / \mathrm{hr}$ by lining our chambers with 5-mil electrolytically deposited copper foil. This 
works, presumably because the deposition filters out the impurities in the copper. We have also tried electrolytically depositing copper on the chambers but with little improvement in background.

We differ from other installations in the type of counting gas that we use. Since 1949 we have used raw methane exactly as it comes from the Gough Well south of Los Angeles. Our reasons are purely economical. We find, however, that there is one difficulty with this gas. When the tank is filled, there is some impurity that condenses at high pressure on the walls of the tanks. When the tanks again reach about $200 \mathrm{lb}$, we begin to notice irregularities in our backgrounds. To avoid this difficulty we disconnect our tanks at $250 \mathrm{Ib}$.

We have tried to compare our methane counting with scintillation counting. In order to get the best geometry, we did not use any thin windows over our phosphors. These phosphors were put on with silicon grease and we found that we had to keep our samples $2 \mathrm{~mm}$ away from the phosphor so that it wouldn't be scratched accidentally. Our geometry was thus 86 per cent of $2 \pi$, and with this geometry our background was $1.8 \mathrm{c} / \mathrm{hr}$. Although we gained on a lower background, we lost on efficiency; therefore, we concluded that the difference wasn't significant enough to justify discarding valuable equipment.

We are operating four Nuclear Measurements Counters, and we find that when the chambers of these counters are new, they have a lower background than the Simpson chamber. However, we use these chambers for counting polonium which, as everyone knows, is quite a volatile substance. Thus, we occasionally contaminate the chambers, and after decontamination we find our backgrounds average $9.0 \mathrm{c} / \mathrm{hr}$ with 50 per cent of a $4 \pi$ geometry. This figure we can maintain quite well.

Electronic malfunctions can contribute quite substantially to background. These troubles are often difficult to locate and require much patience. Two examples will illustrate. Some years ago we were using Glassmike capacitors in the high-voltage supply. If one puts a microammeter in series with the capacitor and $1.5 \mathrm{kv}$ across the combination, some capacitors will show a d.c. leakage of 5 to $10 \mu \mathrm{a}$, whereas others will show no leakage. Replacing these leaky capacitors with good ones will reduce the background. In recent years Glassmikes have improved considerably in this respect.

In Nuclear Measurements Counters we have found that the ceramic disc capacitors which couple the stages of the preamplifier can be a source of trouble. Over a period of months the background will increase steadily. Replacing these capacitors will drop it again. We find that the CornellDubilier brand seems to be best in this respect, but we have never succeeded in eliminating this source of trouble. 
The lack of a good standard in this type of work can be very keenly felt. We have tried to solve our problem in the following way. There are three groups in our Laboratory which do gas-flow counting independent of each other. We therefore had special standards made and took them to each group to be counted. Table 1 summarizes the results of two of the six standards.

\section{Table 1}

$\begin{array}{ccc}\text { Standard } & \text { Group } & \mathrm{c} / \mathrm{m} \\ 1-\mathrm{A} & \mathrm{A} & 21,835 \\ & \mathrm{~B} & 21,986 \\ & \mathrm{C} & 21,941 \\ 2-\mathrm{A} & \mathrm{A} & 12,900 \\ & \mathrm{~B} & 13,132 \\ & \mathrm{C} & 13,003\end{array}$

In all cases backgrounds were negligible and the samples were counted for $30 \mathrm{~min}$. Interestingly enough, the efficiencies quoted varied from 49 to 51 per cent of a $4 \pi$ geometry. We keep these standards under lock and key and check others against them.

\section{Pulse-Height Analysis}

Let us turn our attention now to pulse-height analysis and try to catch up with this fast-moving field. First of all, by a pulse-height analyzer I mean a device which sorts and counts pulses according to their peak voltage. Ideally what we would like to have is a device with an infinite number of channels and one count in each channel. Actually we are limited by the electronics and have to settle on a number of channels which seems reasonable. Before 1946 the single channel was reasonable.

We have two difficulties with analyzers and both relate to channel boundaries. The first difficulty is that if we have a number of channels, each one spaced an increment of voltage above the other, they might drift up and down voltagewise together. If they drift up and down 0.3 volt together, there isn't much point in making the channels less than 0.3 volt wide. Thus, this over-all drift tends to limit the narrowness of the channels 
which you might have. There is an even more serious difficulty in that the channel boundaries might drift with respect to each other, that is, each channel may drift in voltage in a completely random manner independent of its neighbor channels. If we want to count $10^{4}$ random pulses in a channel, we must be sure that during this counting time the drifts in channel widths are to be completely negligible; otherwise, some counts will be dropped into adjacent channels and our statistical accuracy will be destroyed. To give you some idea of what is needed here: If the channel widths are 0.3 volt and you want to count $10^{4}$ random pulses in order to take advantage of the statistics, the channel level should not drift more than $2 \mathrm{mv}$. This is not easy to do in electronics.

About 1946 the trend started to go to 10 channels. These channels were all controlled by multivibrators which were completely independent of each other. Ten-volt-wide channels were popular and it was found that over long periods of time they could be held to 0.25 volt. It soon became apparent, as more and more channels were used, that a disproportionate amount of time was being spent in keeping them "lined up."

In 1950 Wilkinson $^{1}$ at the Cavendish Laboratory published a theory which enabled us to avoid some of these difficulties. The idea was that it is easier in electronics to measure a time than a pulse height. Oscillators can be very stable. Wilkinson's idea was to generate a square wave the duration of which would be linearly related to the pulse height. An oscillator would then be turned on and off by this square wave. Thus, if a pulse were 100 volts high, the duration of the square waves would be just enough to allow 100 cycles of the oscillator. Likewise, if a pulse we re 63 volts high, 63 cycles would be passed from the oscillator. Thus, each cycle would constitute a 1-volt channel and would then be fed to a scaler for summation. If we had a 63-volt pulse and a decimal scaler, the 60 light of the scaler and the 3 light would be turned on indicating that these stages were active. Wilkinson then placed a relay in each stage of the scaler so that it was activated when the stage was on. These relays were then wired in a matrix such that a pulse could be placed in a message register that corresponded to the pulse height. Wilkinson quoted stabilities of 1 per cent over a period of months. We have built eight of these instruments $^{2}$ and found this to be true. Thus, we are not limited by the stability of the instrument. We are limited, however, by the speed of the message registers. These are electro-mechanical devices which in general are not capable of counting pulses faster than 30 times per second. The virtue of the instrument is that it has enabled us to eliminate the problem of drifting channels. Each cycle of the oscillator constitutes a channel and there is no way these channels can overlap. Figure 1 is a picture of this instrument as we have developed it here at Los Alamos. Figure 2 shows 


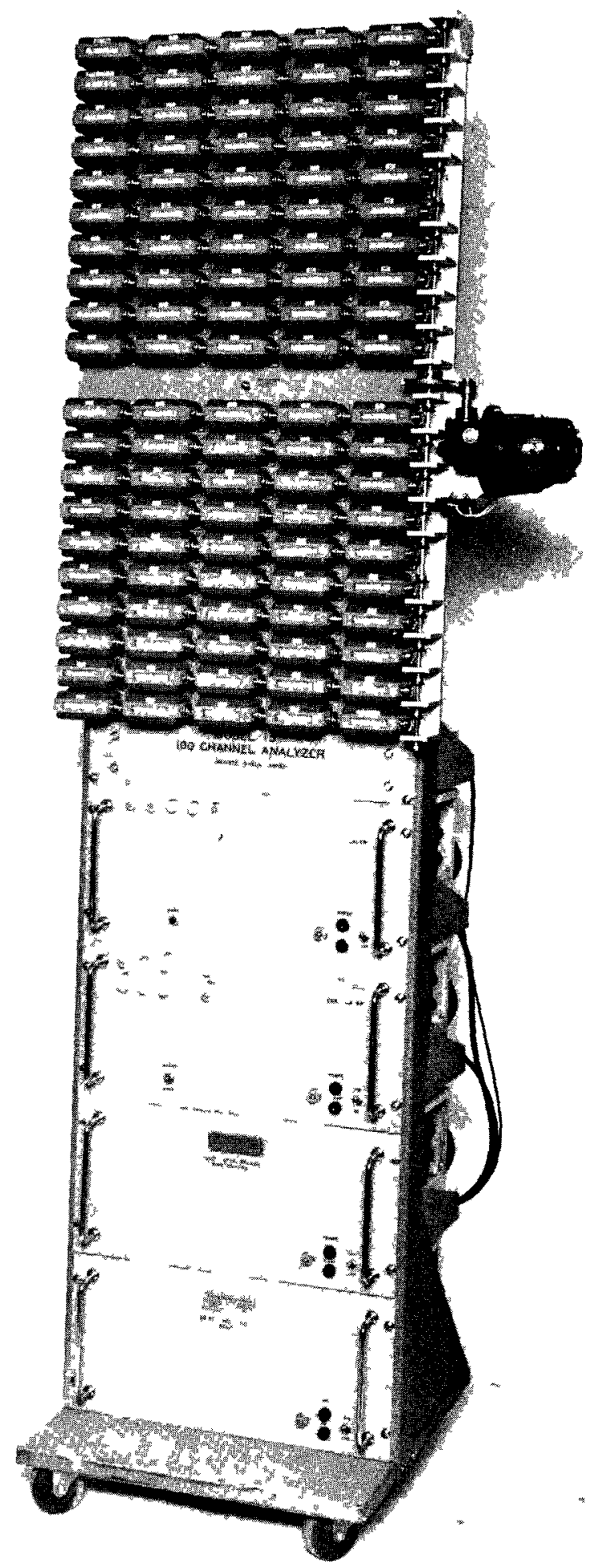

Fig. 1 Model 15100 channel analyzer. 
a typical spectrum taken with a Frisch grid alpha chamber, and Fig. 3 illustrates the over-all linearity obtainable.

The next step was to find some means of speeding up the process. In 1951 Hutchinson and scarrott ${ }^{3}$ published a method for adapting this conversion technique to serial storage using a mercury delay line. Figure 4 illustrates this method as we have developed it at Los Alamos. ${ }^{4}$ Instead of a mercury line we have used a quartz delay line. The pulse to be analyzed is first "stretched." That is, the amplitude is preserved accurately, but the pulse is made to have a much longer duration. In this case the duration is much longer than $990 \mu \mathrm{sec}$, which is the time required for a pulse to travel around the delay line. If we can imagine a fiducial pulse to be traveling around the delay line, then we can imagine that this pulse, when it passes a point in the delay line, will start a sawtooth sweep voltage. The flducial pulse will, of course, continue on its way. We can arrange a circult so that when the amplitude voltage of the sawtooth sweep is identical to that of the stretched pulse, a second pulse will be inserted into the delay line. Thus, the time at which this second pulse is inserted into the delay line after the fiducial pulse is a measure of the pulse height.

Unfortunately, it is not possible to do the conversion quite this simply because another pulse of the same height could cause a third pulse to be inserted at the same time as the second. Thus, it is necessary to divide the line into channels with an oscillator. The actual number of the pulse is then stored in the line. Inasmuch as we are not electronic specialists, it seems advisable to back away from the electronics at this point. Figure 5 illustrates what the contents of this memory look like when they are presented on an oscilloscope. The horizontal lines represent the separation of the digits; thus, the lowest four vertical dots represent the first digit of the number. A channel is represented by all the dots in a vertical line. Typical performance is a 1 per cent loss at 20 pulses per second.

Our contribution to this instrument was to store the numbers in this coded decimal form instead of binary, and then to arrange the output of the machine so that the number in each channel could be printed out on an adding machine. There is a great deal of interest in analyzers of this type at present. Many universities are working on them and they appear to have a good future. We have bullt three; however, since the development of magnetic cores, our interest has turned to their possibilities.

The magnetic-core storage instruments ${ }^{5}$ are considerably more expensive than the delay-line type since more components are required. The advantage of the magnetic core is that it is possible to store numbers more rapidly than in a delay line. We have seen that the delay line stores in a serial fashion. The magnetic core stores in a parallel fashion. This is essentially the difference between the two machines. Both use the 



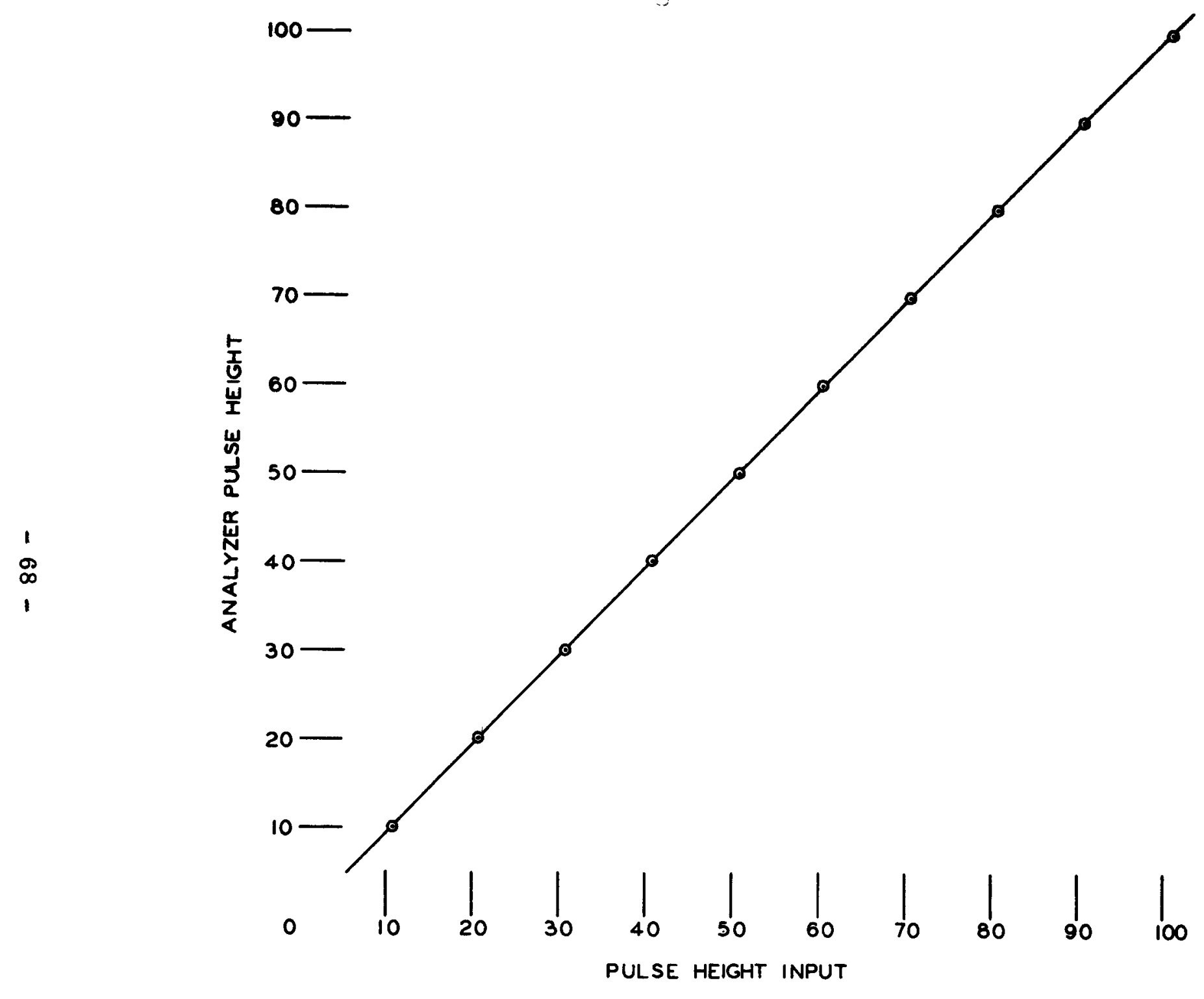

Fig. 3 Over-all linearity of the Model 15100 channel analyzer. 

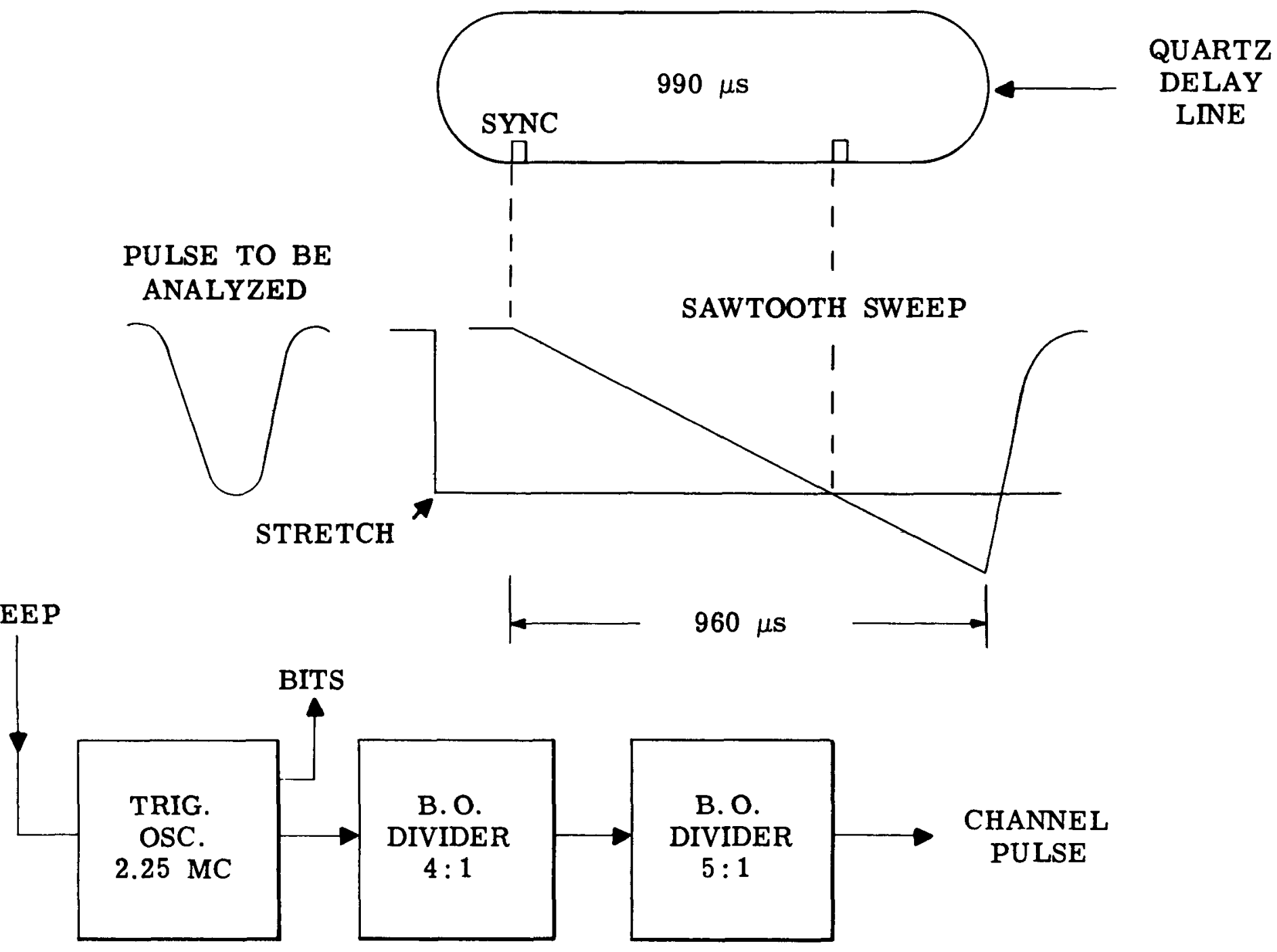

Fig. 4 A simplified schematic of the principle of operation of the modified Hutchinson-Scarrott type of analyzer. 


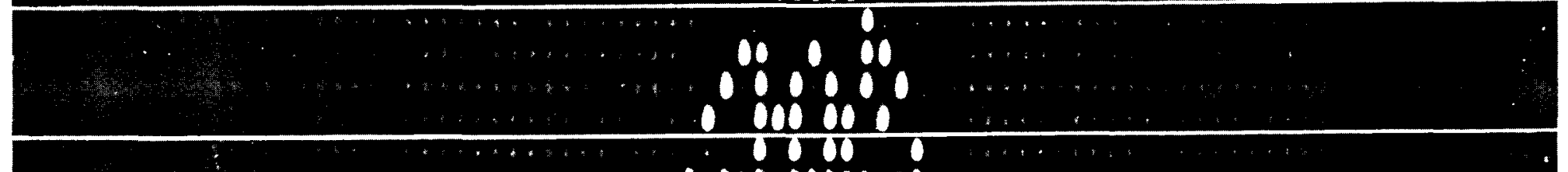

carmen

Prit

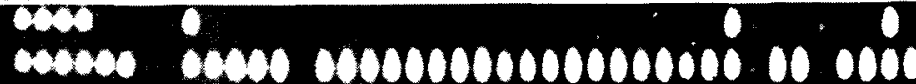

cromen

- o conco

$\sin 0000000000$

000000

000000000

$00,0000,00$

w os ovos 0000

- cos cos o

$\infty .00$

800

00000000000000 o : 800000

0.00

100

00000000

000000

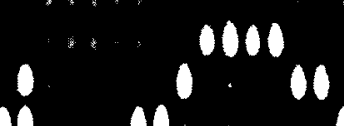

0 1

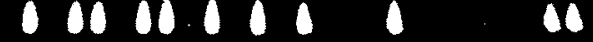

ose nos sose

Fig. 5 A photograph of an oscilloscope presentation in the coded decimal form. 
principle of pulse-helght-to-time conversion. Figure 6 illustrates the characteristics of the core. We can draw the hysteresis loop as a function of $I$, the current through the cores. These cores have the following property: If a current, $I$, is put through them, they will revert to the opposite state. To do this the polarity of I will depend on the state the core is in. If the core is in state 1 , a positive current will switch it to state 0 . Likewise, if it is in state 0 , a negative current will switch it to state 1 . Currents of the opposite polarity to the one required have little effect. Another remarkable property of these cores is that if a current $I / 2$ is put through them, there will be little effect even if it is of the correct polarity. In fact, an infinite number of $I / 2$ pulses may be put through a core without switching it to the opposite state.

If another winding, called a sense winding, is threaded through the core, a signal will be induced in it if the core changes to the opposite state. Thus, as illustrated in Fig. 6, if the core was in the 1 state, a positive voltage, as shown, will be induced if a positive current, $I$, is pulsed through it. In the zero state a positive current will induce a much weaker response. In the zero state the voltages induced will be of the opposite polarity; however, they can be readily reversed with electronic circuitry. These cores are wired up in a $10 \times 10$ matrix as lllustrated in Fig. 7 . Thus, if $I / 2$ is placed on each bus, only the intersecting core will see I and the sense winding will have a response attributable to that core. Our scaler is then connected to this matrix, and again we are able to select a core depending on the number in the scaler. The planes are stacked up 20 high and wired together in series. Thus, the scaler will select one core in each plane. Each plane, however, has its own sense winding which is connected to each stage of a second scaler. The reason for this is that we cannot store the number directly in the core. The old number must first be telegraphed out of the core in which it was stored and into the second scaler. We then add one to this scaler which gives our new number and this number is then telegraphed from the second scaler into the store. This process requires about $25 \mu \mathrm{sec}$. As you can imagine, it requires somewhat elaborate circuitry and Fig. 8 illustrates its complexity. The core planes are in the middle surrounded by the second scaler and other circuitry. Figure 9 shows a picture of the completed instrument. With these faster techniques we are now able to count 1000 pulses per second with a 1 per cent loss. These conversion type instruments are still slow compared to the older type instruments which used independent discriminators and scalers. However, the stability plus the number of channels is far superior to the older 10channel instruments. It is not uncommon in our experience to hear a physicist say that he can accomplish as much in one-half hour with these instruments as he could all day with the 10-channel instruments. 

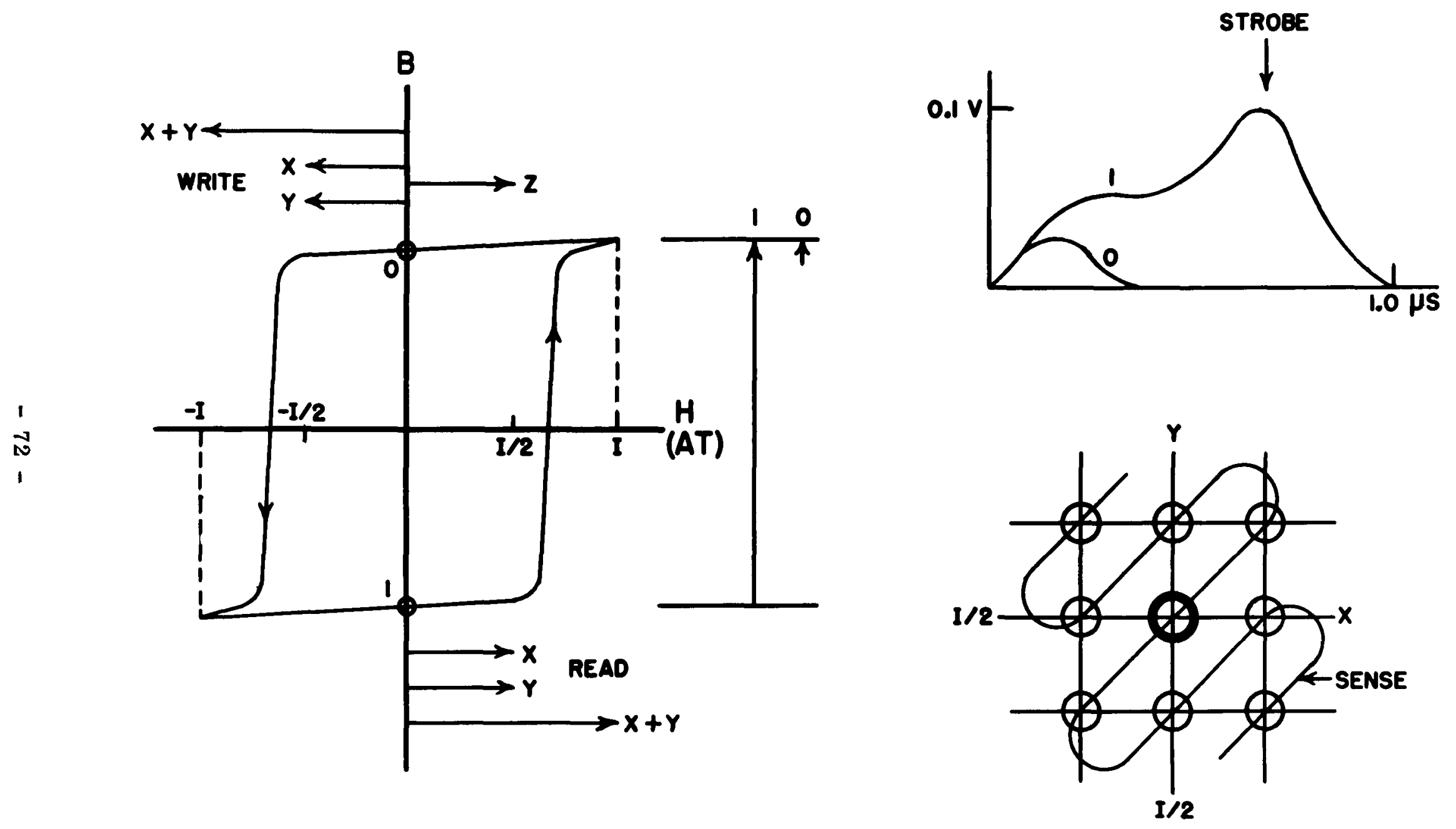

Fig. 6 Magnetic core characteristics. 


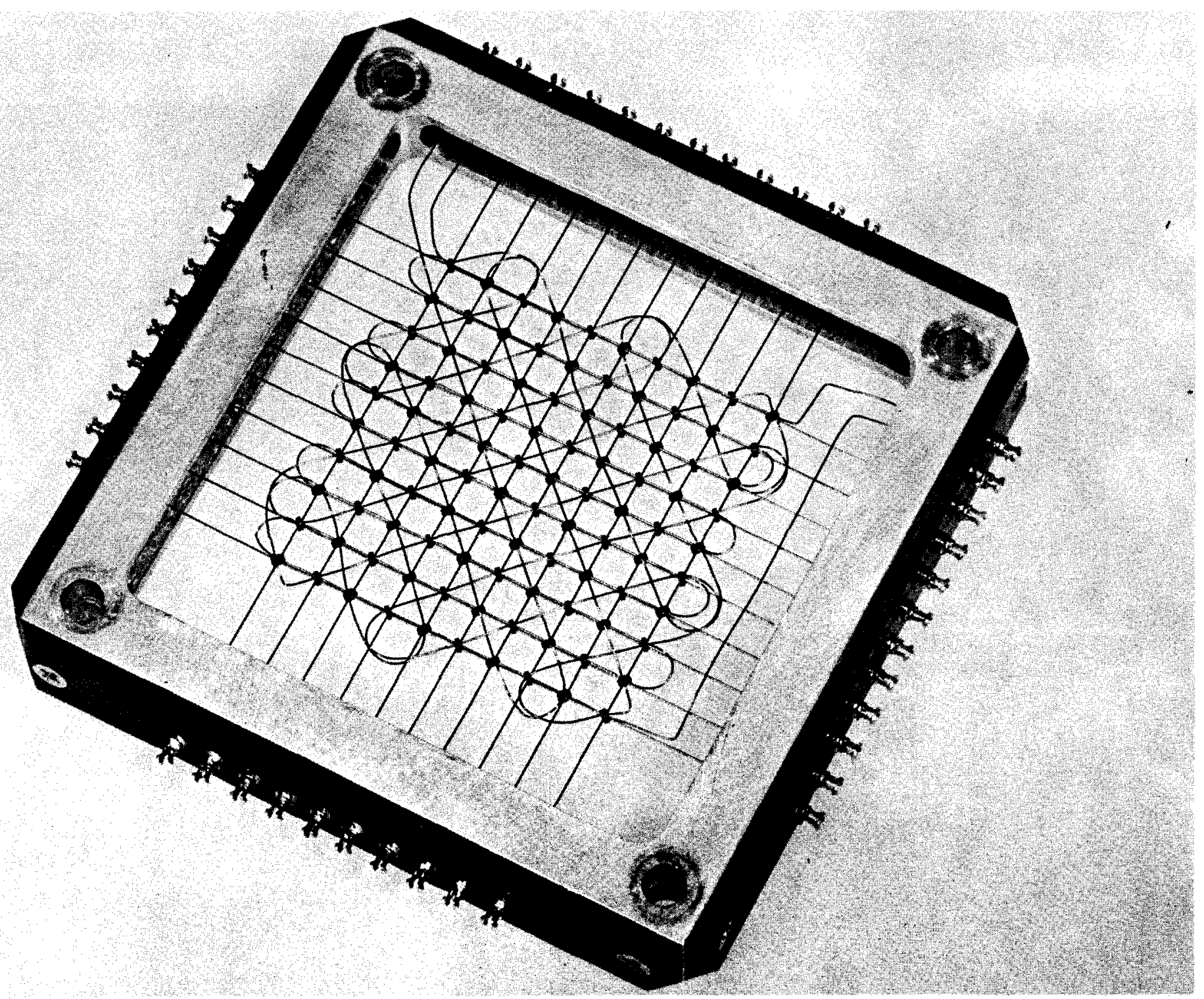

Fig. 7 A typical magnetic memory core plane. 


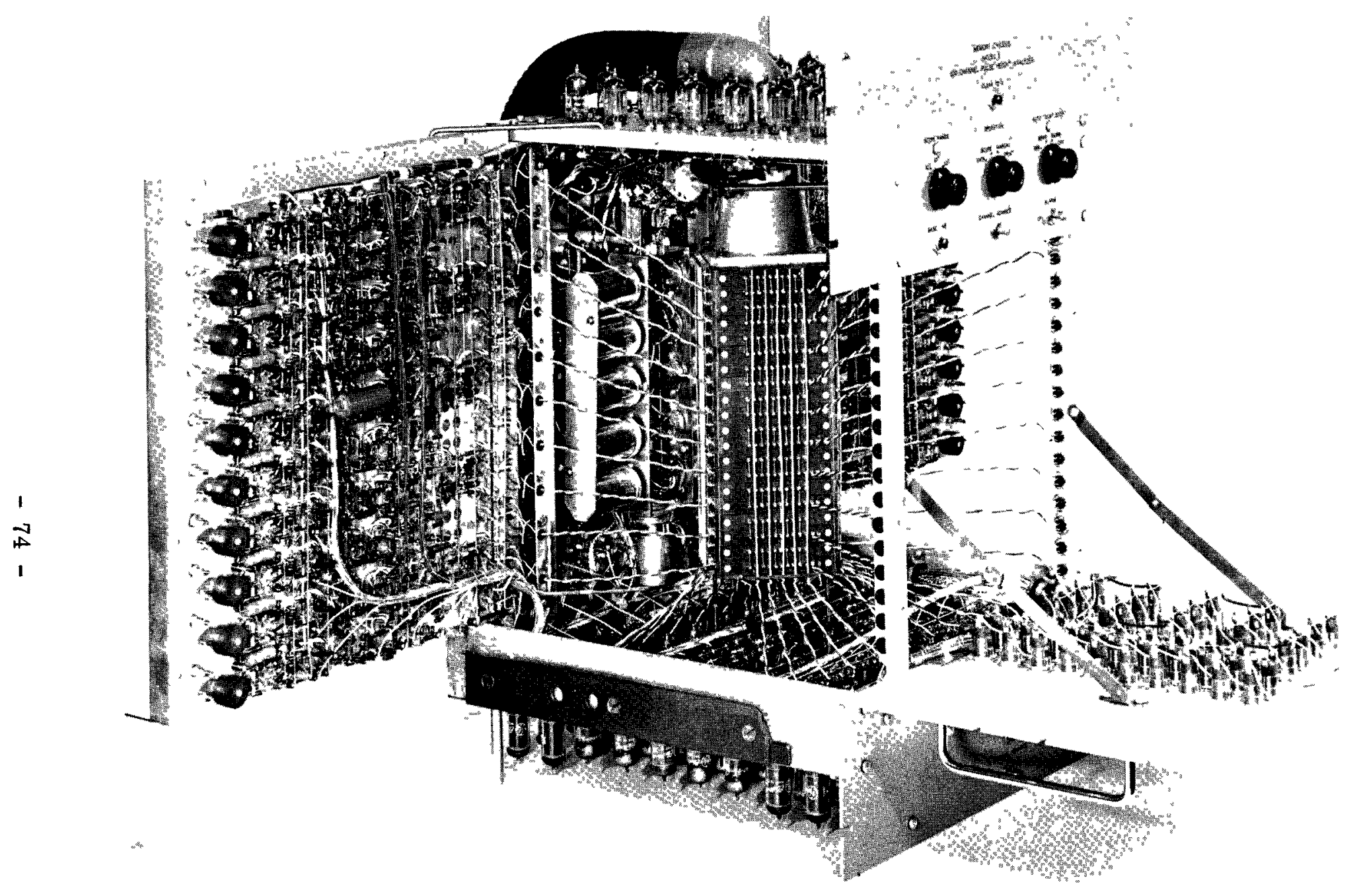

Fig. 8 Memory chassis opened for servicing. 


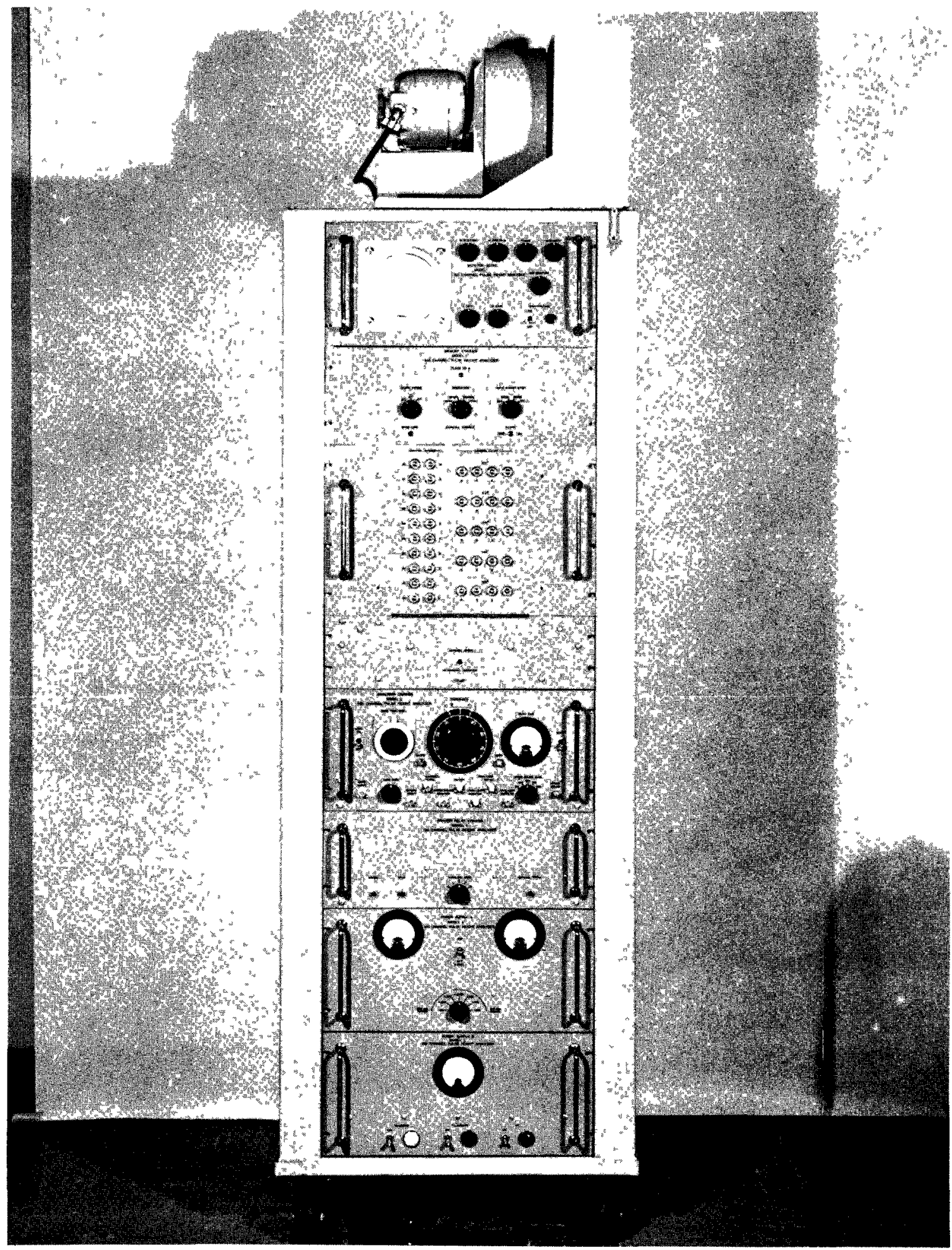

Fig. 9 Front view of the analyzer. 
This speeding up of data gathering has again introduced new problems. There is a considerable trend toward taking the output of these machines and punching tabulating cards directly. With these instruments it is possible to do the following: (a) deposit the contents of the memory on magnetic tape to be fed into a computer, (b) print the number in each channel on adding machine paper, (c) punch the numbers in code form on a tape, and (d) draw an analog curve of the numbers in the whole 100 channels. Other trends are: (a) the transistorizing of the magnetic memory, (b) the desire for less complexity with a consequent gain in reliability, and (c) the desire for faster machines. There is little doubt that these objectives will be attained in the next few years.

\section{References}

1. Wilkinson, D. H., "A Stable Ninety-Nine Channel Pulse Amplitude Analyser for Slow Counting," Proc. Cambridge Phil. Soc., 46, 508-518 (1950).

2. Hiebert, R. D., "The Model 15100 Channel Pulse Height Analyzer," Los Alamos Scientific Laboratory, Report LA-1565, April 1953.

3. Hutchinson, G. W., and Scarrott, G. G., "A High Precision Pulse Height Analyser of Moderately High Speed," Phil. Mag., 42, 792-806 (1951).

4. McKibben, J. L., Gallagher, J. D., and Lang, H. J., "Modifications to the Hutchinson-Scarrott Pulse Height Analyzer to Obtain Coded Decimal Presentation and a Decimal Print-Out," IRE Convention Record, Part $10,186-190,1955$.

5. Byington, P. W., and Johnstone, C. W., "A 100-Channel Pulse Height Analyzer Using Magnetic Core Storage," IRE Convention Record, Part 10, 204-210, 1955. 
PANEL DISCUSSION OF PLUTONIUM

Wright H. Langham, Los Alamos Scientific Laboratory

In the 15 minutes which has been allotted for this talk, I should like to mention a few of the problems encountered in the control of plutonium health hazards and to ask the panel to consider them. I should like to begin by talking about the basis on which the maximum permissible level for plutonium is derived.

- The maximum permissible level (MPL) for plutonium is considered to be $0.5 \mu \mathrm{g}(0.04 \mu \mathrm{c}$ as it is usually rounded off in the handbooks of the National and International Commissions for Radiological Protection). Maximum permissible levels, in general, are derived from three different sources. First, MPL's for radionuclides that deposit predominantly in bone are derived from a comparison with radium. We have had sufficient experience with radium to conclude that $0.1 \mu \mathrm{g}$, fixed in the body, supposedly will do no bodily harm. On the basis of this one point, established from human experience, the International Commission of Radiation Protection, as well as the National Commission on Radiological Protection, has decided to relate all bone seekers to radium according to the following equation:

$$
(\mathrm{MPL})_{\mathrm{n}}=\mathrm{Q}=\frac{0.1 \times 0.99 \times[\Sigma \mathrm{E}(\mathrm{RBE}) \mathrm{N}]_{\mathrm{Ra}}}{\mathrm{f}_{2} \times[\Sigma \mathrm{E}(\mathrm{RBE}) \mathrm{N}]_{\mathrm{n}}}
$$

In this equation $Q$ is the MPL of the nuclide, $n$, expressed as microcurtes and is that amount of material which will deliver to the bone a radiation dose equivalent in biological effect to that of $0.1 \mu \mathrm{g}$ of radium; $f_{2}$ is the fraction in the bones of that in the total body (99 per cent for radium): $\Sigma E(R B E) N$ is a weighted energy term, weighted for the average energy of each type of radiation emitted in the decay chain, their respective RBE's, (relative biological effectiveness) and the fraction of the total energy, $N$, 
absorbed in the bone. This term for $\mathrm{Ra}^{226}$ (assuming 50 per cent of the radon is exhaled before it can undergo further decay) equals 16 . This expression then becomes

$$
\mathrm{Q}=\frac{16}{\mathrm{f}_{2} \times[\Sigma E(\mathrm{RBE}) \mathrm{N}]}
$$

The RBE of alpha particles, by virtue of committee decision, is set at 10 . The quantity $\mathrm{N}$ is sometimes called the "coefficient of ignorance" since it also includes a safety factor to allow for nonhomogeneity of distribution in the critical organ. For example, it is not known whether the mode by which plutonium deposits in the body is more harmful than that of radium. Radium incorporates in the inorganic salts while plutonium seems to be confined to the inorganic or organic surfaces. For plutonium $\mathrm{N}$ has been given a value of 5 which says that the method by which plutonium deposits in the body is 5 times more harmful than that of radium, $f_{2}$ is 75 per cent, the $R B E$ is 10, and the energy is $5.15 \mathrm{Mev}$. Substituting in equation (2), the MPL for plutonium becomes

$$
\mathrm{Q}=\frac{16}{0.75 \times 5.15 \times 10 \times 5}=0.08 \mu \mathrm{c},
$$

which is twice the level in the handbooks.

Maximum permissible body burden may also be derived from human experience with $x$-ray. A committee of authorities has said that, based on our experience with $\mathrm{x}$-ray, $0.3 \mathrm{r} /$ week of hard $\mathrm{x}$ - or $\gamma$-radiation will not, in the course of a working lifetime, produce an appreciable or significant bodily injury. The MPL's of internal emitters can be calculated in relation to this value. The following expression is the one given by the International Commission of Radiological Protection:

$$
\mathrm{Q}=\frac{(100 \mathrm{ergs} / \mathrm{g} / \mathrm{rad})(\mathrm{W})(\mathrm{M})}{\left.\left(3.7 \times 10^{4} \mathrm{~d} / \mathrm{s} / \mu \mathrm{c}\right)\left(1.6 \times 10^{-6} \mathrm{erg} / \mathrm{Mev}\right) ; 6.05 \times 10^{5} \mathrm{~s} / \text { week }\right)\left(\mathrm{f}_{2}\right)[\Sigma \mathrm{E}(\mathrm{RBE}) \mathrm{N}]}
$$

In this expression $Q$ is the microcuries of the substance in question; $W$ is the dose, $0.3 \mathrm{r} /$ week; $M$ is the mass of the critical tissue in which the substance is predominantly deposited; $f_{2}$ is the fraction of the material in the total body that is deposited in the critical tissue of $\mathrm{M}$ grams; $\Sigma \mathrm{E}$ is the 
summation of the energies of the disintegrations; $R B E$ is the relative biological effectiveness; and $\mathrm{N}$ is again the factor for nonhomogeneous distribution and absorption of the radiation in the critical organ. If we substitute $0.3 \mathrm{r} /$ week for $\mathrm{W}, 10,000 \mathrm{~g}$ of bone for $\mathrm{M}, 0.75$ for the fraction of the plutonium deposited in bone, $5.15 \mathrm{Mev}$ for $\Sigma \mathrm{E}, 10$ for the $\mathrm{RBE}$, and 5 for $\mathrm{N}$, we have $Q$ equal to $0.04 \mu \mathrm{c}$ of plutonium as the total body burden. There is an interesting point about this particular calculation. If we use the same calculation to obtain the maximum permissible level for radium, even using $\mathrm{N}$ equal to 5 , we get $0.27 \mu \mathrm{c}$ as the MPL for radium. This is nearly a factor of 3 higher than the officially accepted value.

The third method for obtaining the MPL's is to obtain them directly from biological or animal data. This has been done for plutonium and an account of some of the work is of interest. Austin Brues and Mariam Finkel at Argonne set up experiments on mice in which they compared, on the basis of injected dose, the effect of radium and plutonium on tumor incidence. They arbitrarily assigned radium a biological effect of 1 and compared the effect of plutonium to radium. The dose of radium required to produce a given tumor incidence was determined experimentally. The tumor incidence produced by varying dosages of plutonium was also determined. They found that plutonium had an RBE of 30 compared to radium in the dose ranges which produced a 60 per cent tumor incidence in their animals. As the work continued, it was found that the RBE decreased as the tumor incidence decreased. At present the RBE for plutonium is about 5 for the production of less than 10 per cent tumors. This is approaching the area in which we are interested since we want to find that dose which will produce a minimal number of tumors in man. As the dose-response curve in Brues and Finkel's experiments tends to flatten out in the region of low tumor incidence, it may be reasonable to assume that (RBE)N for plutonium, compared to radium, is about 5 .

Other work was done to compare the life shortening of mice by plutonium as compared to radium. In the region of less than 1 to 10 per cent life shortening, the RBE of plutonium may actually be as low, or lower, than that of radium. On the basis of these experiments and other results relating injected dose to retained dose and correcting for the amount of radon exhaled by mice as compared to man, the MPL for plutonium was officially set at $0.04 \mu \mathrm{c}$. Fortuitously, the biological value agrees with that obtained from the tolerance value for $\mathrm{x}$ - or $\gamma$-radiation, $0.3 \mathrm{r} /$ week. Various work done since this time indicates that the MPL could be raised while still other work shows that it should be lowered. At present the MPL of $0.04 \mu \mathrm{c}$ of plutonium still looks like a reasonable value.

The bio-assay work with which most of us are involved attempts to determine the body burden by relating the amount of material excreted to 
the amount retained in the body. We can analyze feces, urine, and blood. If we can establish the correlation between the urine, fecal, or blood elimination rate and the amount retained in the body, then we can determine the body burden of an individual. The problem, then, is to establish a relationship between excretion and the body burden. This can be done with animal or human experimentation. It cannot be done by analyzing the excreta from accidental exposures. In these cases one can get the slope, or slopes, for the excretion curve but not the absolute values since one does not know the amount of material the individual has absorbed.

Both animal and human experimentation have been done to obtain the per cent excretion as a function of body burden, or the retention in terms of the excretion rate. Fifteen hopelessly sick individuals were given very small single injections of plutonium intravenously as the citrate, and their urine, feces, and blood were analyzed as a function of time after administration. The work showed that urine was the excrement, or fluid of choice, for analysis. The blood values dropped too low to be detected unless large volumes of blood were taken, and the feces presented well-known analytical problems. When a semi-log plot is made of per cent of injected dose excreted per day in the urine versus time after injection, one finds that on the first day about 0.8 per cent was excreted, in 5 days the excretion value dropped to 0.1 per cent, in 40 days it was 0.01 per cent, and at 1200 days it dropped to 0.001 per cent of the body burden. This is not a straight line on a semi-log plot but can be expressed by the following power function:

$$
\mathrm{Y}_{\mathrm{u}}=0.002 \mathrm{t}^{-0.74}
$$

This excretion curve may be in error by as much as 20 to 30 per cent, but it is essentially the only information available on humans in which the body burden was known. This power function can be expressed by four simple exponentials, and one can postulate the reservoir which is the controlling factor in determining the slope for each of the exponentials. For instance, one can postulate that the very long turnover times indicated by the curve represent excretion from the bone.

Now I should like to outline some of the problems that arise in using the above data to control the plutonium health hazards in a plant operation. It would appear that if we have these data, we can analyze a 24-hr sample of urine and, knowing the date of exposure, immediately determine the body burden. It is not quite that simple. There are a number of factors involved which one must keep in mind. First, the data were not obtained from normal people, although we do not think that this greatly affected the results. When 
these data were compared with those obtained from young healthy individuals who had had accidental exposures at Los Alamos, we found that the slopes of the excretion curves were quite comparable. Secondly, these data were obtained from single intravenous injections. An intravenous injection is certainly not comparable to the situation one faces in plant operations. In other words, does a curve obtained from intravenous injection give us a basis from which to determine exposure by other means? A third question is: Can an excretion curve determined on the basis of a single exposure be used as a basis for determining the body burden of an individual who is receiving multiple exposures? A fourth question 1s: What type of sample can we take which will most adequately allow us to use these data in determining a body burden? Do we take a 24-hr sample; do we take two morning voidings; two evening voidings; or do we take the voiding during the first three hours following an exposure, etc.? The flfth question concerns the medical implications and management. If we have an accident resulting in a highly contaminated individual, do we treat him; how and when? Do we decontaminate; if so, how and when? If we find he has received a MPL, do we retire him from further work (since excretion of 50 per cent of his body burden will require about 200 years); and if we do, how do we do it without legal obligation?

With this introduction, which gives many of the toughest problems facing us but gives none of the answers, I would like to turn the meeting back to the panel.

J. W. Healy, Hanford Atomic Products Operation

The problem which I plan to discuss is that of plutonium in the lung. Actually, of all the types of human exposure to plutonium, the most probable is inhalation, but the fate of plutonium after it gets into the lung is not known. I would like to present some evidences of its disposition which we have today. For the purposes of these calculations one should consider the lung apart from the body. In other words, it is separated from the body, so to speak, by a membrane so that urinalyses do not show what is actually in the lung. Hence, estimates of the amount of plutonium in the lung must be made by secondary methods.

If one examines an actual plot of the excretion rate of an individual who has had an inhalation exposure to insoluble plutonium, one finds that the data 
are not fully described by Dr. Langham's equations, * which were derived from intravenous injections of soluble plutonium. The rate falls for a few days as expected and then actually increases from 10 to 200 days. We postulated that such excretion rates could be approximated by assuming a chronic administration of a small amount of plutonium from the lung to the body each day.

As the plutonium is eliminated from the lung, the size of the metabolic pool, and thus the rate at which the plutonium enters the blood stream, decreases. We assumed that the rate of transfer from the lung to the blood stream is essentially a linear function of the quantity present in the lung; or in other words, the quantity in the lung decreases according to an exponential. Then the quantity of plutonium remaining in the lung, $Q$, at a time, $t$, following an acute fixation of a quantity, $Q_{0}$, of an insoluble compound is given by the equation

$$
Q=Q_{0} e^{-\lambda t}
$$

In this equation the over-all rate of elimination is taken as $\lambda$. This rate represents the sum of the rates of the two mechanisms by which plutonium is assumed to be removed from the lung: (1) solubilization and transfer to the blood stream, $\lambda_{\mathrm{s}}$, and (2) ciliary action, $\lambda_{\mathrm{c}}$. Note that $Q_{0}$ includes only that plutonium retained in the lung after initial clearance, i.e., immediately following exposure, a large amount of plutonium is deposited in the bronchial tree and is rapidly eliminated by ciliary action. The rate of transfer, $A$, of the plutonium in the lung to the blood stream is then given by

$$
A=Q \lambda_{S}
$$

If the urinary excretion rate resulting from each increment of this administration is described by the Langham equation

$$
x_{u}=0.002 t^{-0.74}
$$

\footnotetext{
* Langham, W. H., "Determination of Internally Deposited Radioactive Isotopes from Excretion Analyses," Am. Ind. Hyg. Asso. Quart., 17, 305-318 (1956).
} 
the total excretion can be considered as the sum of the excretion rates from the incremental steps. In setting up this equation, $t$ is the time since the material was absorbed into the blood stream. If $R$ is the time at which analyses are taken following entrance of the material to the lung, and $E_{u}$ is the resulting rate of excretion, then

$$
E_{u}=0.002 \lambda_{s} Q_{0} \int_{0}^{R} e^{-\lambda t}(R-t)^{-0.74} d t
$$

Equation 4 is not integrable and must be solved for individual values of $\lambda$, $R$, and $t$. This has been accomplished by expansion of the exponential term and solving until the series converges. Figure 1 shows the solutions of the integrals for various values of $\lambda$ and $t$.

We can also carry out the same type of calculation for fecal excretion using the Langham equation for fecal excretion of plutonium. We can estimate the quantity of plutonium which should be found in the feces due to the plutonium transferred chronically from the lung to the blood. These results can then be compared with those obtained from actual fecal analyses.

These considerations have been applied to several cases of plutonium deposition in which the excretion curves deviate from those expected for a single administration. Figure 2 shows such an excretion curve in a formalized manner. We assume that the early excretion is due to the initially soluble plutonium fraction which is absorbed into the body quite rapidly and behaves as a single administration. This portion of the curve shows a rapid decrease. The total curve is the sum of the lung contribution plus that from the initial soluble fraction. Actual data are shown on the curves in Fig. 3. The plutonium excretion is high at first and then seems to level off, dropping as one would expect. From the shape of the curve we estimate $\lambda$ to be on the order of 0.001 per day, or, approximately a two-year half-life.

Table 1 shows some estimates of the half-life made from several cases with low-level excretion rates. There appears to be a correlation between the total time for which we have analyses and the value of the halflife in days. The longer we examine urine specimens following an exposure, the longer the half-life appears to be. This may be a function of our curvefitting, which is difficult with the scatter in the data. 


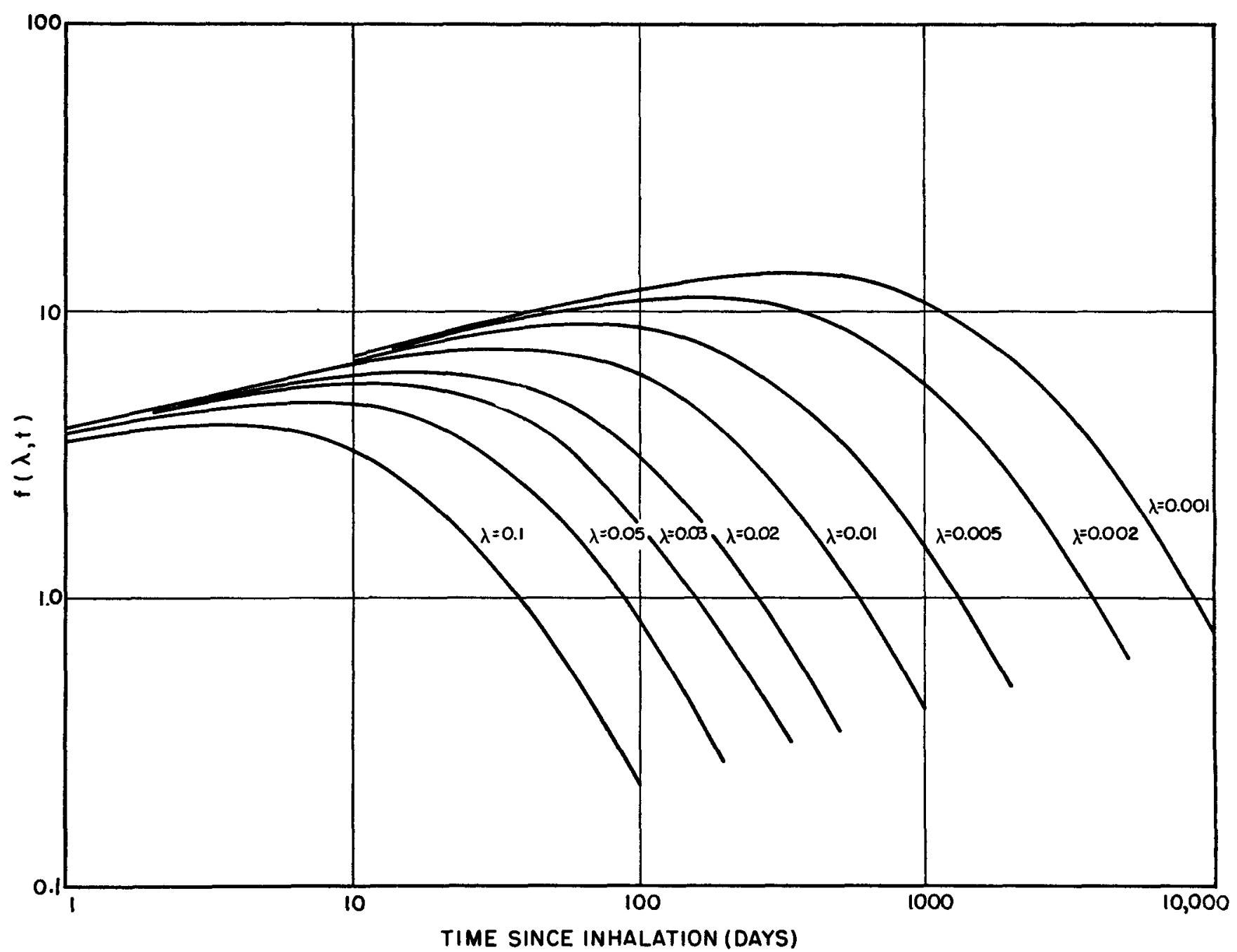

Fig. 1 Urinary excretion of plutonium derived from lung transfer, where $E_{\dot{u}}^{\prime}=0.002 Q_{o} \lambda_{s} f(\lambda, t)$ and $f(\lambda, t)=\int_{0}^{R} e^{-\lambda t}(R-t)^{-0.74} d t$ 


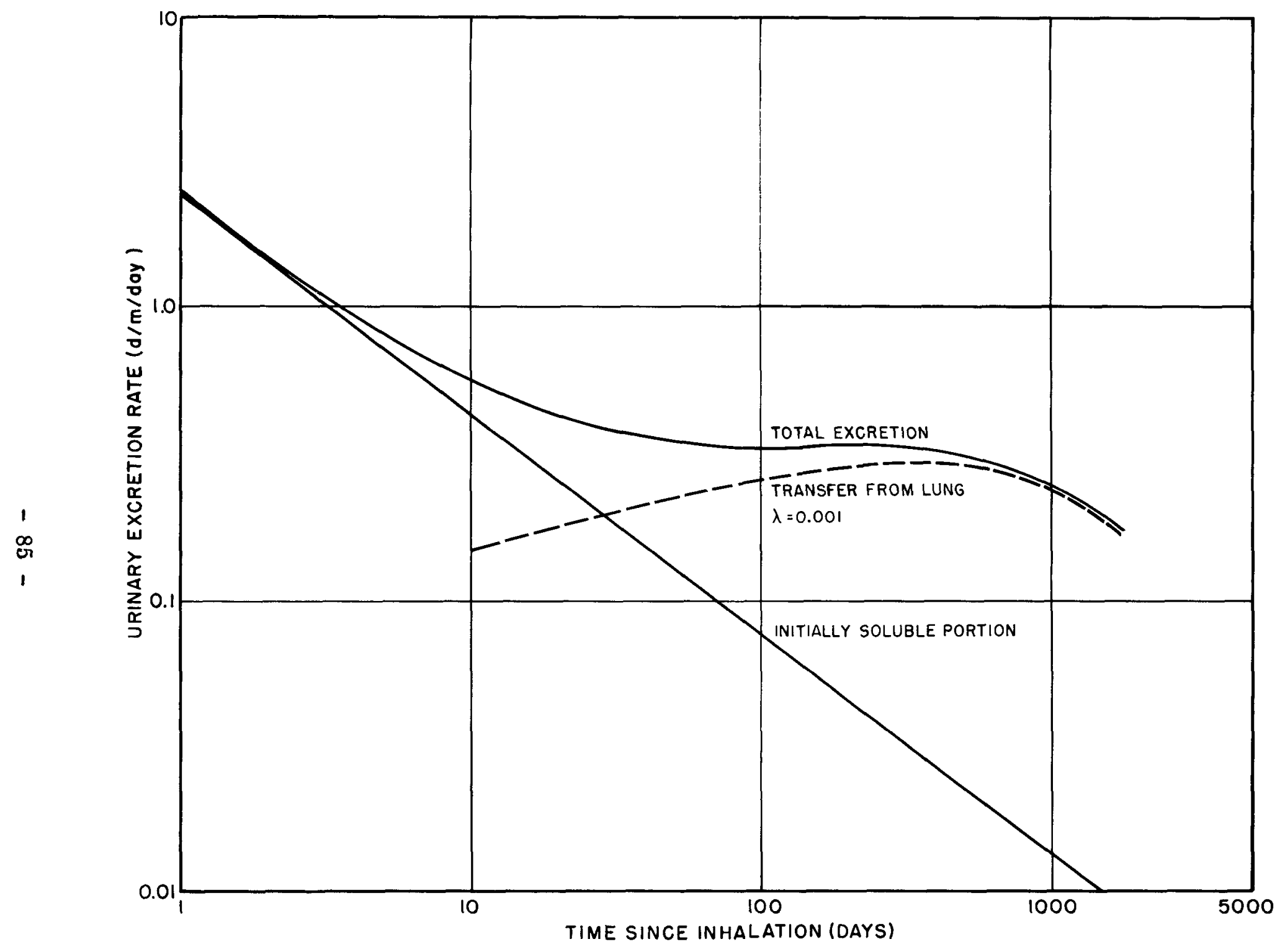

Fig. 2 Urinary excretion of $\mathrm{Pu}^{239}$ in a lung deposition case. 


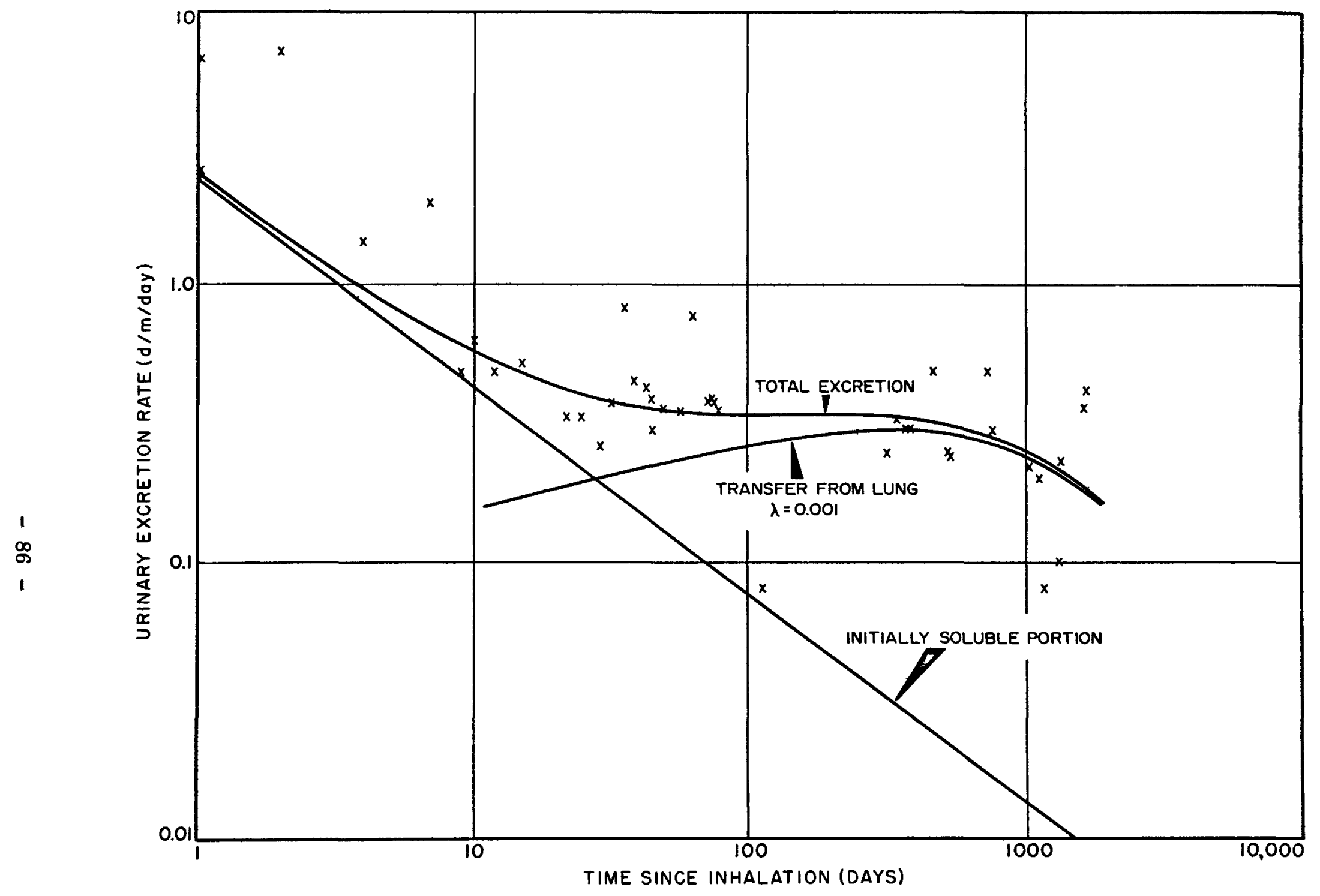

Fig. 3 Urinary excretion of $\mathrm{Pu}^{239}$ in a lung deposition case showing actual data from which curves were plotted. 
Table 1

ESTIMATED PLUTONIUM TURNOVER RATES IN THE LUNG

Case

$\begin{array}{lr}\text { A } & 235 \\ \text { B } & 435 \\ \text { C } & 455 \\ \text { D } & 475 \\ \text { E } & 480 \\ \text { F } & 655 \\ \text { G } & 965 \\ \text { H } & 1660\end{array}$

Time Examined, days

235

435

455

475

480

655

1660
Half-Life, days

$\lambda /$ day

0.01

0.003

0.005

0.003

0.005

0.005

0.003

0.001
70

230

140

230

140

140

230

700

In Table 2 I have calculated the maximum excretion rate for $0.016 \mu \mathrm{c}$ of plutonium initially deposited in the lung and tabulated the approximate time at which the maximum excretion should occur. You will note that the excretion rate is not high, even at the maximum.

\section{Table 2}

MAXIMUM EXCRETIONS FOR $0.016 \mu \mathrm{c}$ OF Pu IN THE LUNG

\begin{tabular}{|c|c|c|c|c|}
\hline $\begin{array}{l}\text { Half-Life } \\
\text { in Lung, } \\
\text { days }\end{array}$ & $\lambda /$ day & \begin{tabular}{l}
\multicolumn{1}{c}{ Time of } \\
Max. Excretion, \\
days
\end{tabular} & $\begin{array}{l}\text { Value of } \\
f(\lambda, t)\end{array}$ & $\begin{array}{r}\mathbf{E}_{\mathrm{u}}^{\prime}, \\
\mathrm{d} / \mathrm{m}\end{array}$ \\
\hline 70 & 0.01 & $30-50$ & 7.2 & 5.1 \\
\hline 140 & 0.005 & $\sim 50$ & 8.7 & 3.1 \\
\hline 350 & 0.002 & $\sim 100$ & 11 & 1.6 \\
\hline 700 & 0.001 & $\sim 500$ & 13 & 0.9 \\
\hline
\end{tabular}

We have applied the same type of analysis to feces data. Table 3 shows the results obtained in one case in which we had both urine and feces analyses. 
Table 3

FECES DATA

$\begin{array}{ccccc}\begin{array}{c}\text { Time since } \\ \text { Exposure, } \\ \text { days }\end{array} & \begin{array}{c}\text { Calc. Total } \\ \text { Fecal Excretion, } \\ \mathrm{d} / \mathrm{m} / \text { day }\end{array} & \begin{array}{c}\text { Measured } \\ \text { Fecal Excretion, } \\ \mathrm{d} / \mathrm{m} / \text { day }\end{array} & \begin{array}{c}\text { Ciliary } \\ \text { Contribution, } \\ \mathrm{d} / \mathrm{m} / \text { day }\end{array} & \begin{array}{c}\text { Rate of } \\ \text { Solution, } \\ \mathrm{d} / \mathrm{m} / \text { day }\end{array} \\ 40 & 0.39 & 1.8 & 1.4 & 11 \\ 70 & 0.35 & 3.5 & 3.1 & 10 \\ 280 & 0.30 & 1.0 & 0.7 & 8.4 \\ 281 & 0.30 & 0.9 & 0.6 & 8.4\end{array}$

The difference between the calculated and measured fecal excretion was attributed to the long-term movement of plutonium from the lung by cillary action and subsequent elimination through the GI tract. Comparing the quantity of plutonium estimated to move from the lung to the body per day with the measured daily fecal excretion rate, we see that roughly 10 per cent is eliminated through the feces and 90 per cent is absorbed into the body. Another case in which we analyzed feces samples at 460 days shows approximately the same ratio. The estimated rate of solution in this case was about $55 \mathrm{~d} / \mathrm{m} /$ day, with a ciliary contribution of about 4 to $8 \mathrm{~d} / \mathrm{m} /$ day. We also attempted to fit a power function to the blood retention of plutonium using the data given in Dr. Langham's report. This gave us an equation for retention in the total blood:

$$
\mathrm{X}_{\mathrm{B}}=0.29 \mathrm{t}^{-1.32}
$$

With this exponent the quantity of plutonium in blood decreases rapidly. Blood samples from one individual gave $211 \mathrm{~d} / \mathrm{m}$ at 140 days and $188 \mathrm{~d} / \mathrm{m}$ at 220 days, assuming the blood volume for the standard man. Since these results were high compared to the quantity one should find in the blood on single administration, we integrated over this time period to obtain the blood plutonium which would result from a chronic administration from the lung. We estimated the lung burden from the urine curves as described earlier. We calculated that at 140 days one should have $231 \mathrm{~d} / \mathrm{m}$ and at 220 days, $162 \mathrm{~d} / \mathrm{m}$. The agreement may be fortuitous, but it indicates that the movement of plutonium to the blood stream is occurring at about the rate expected. In another case we measured $16 \mathrm{~d} / \mathrm{m}$ in the blood at 872 days and calculated $8 \mathrm{~d} / \mathrm{m}$, and at 955 days we measured $7 \mathrm{~d} / \mathrm{m}$ and 
calculated $6 \mathrm{~d} / \mathrm{m}$. These results are of the order of magnitude that one would expect, so we can conclude that this method of interpretation at least is consistent.

\section{Questions and Answers}

Question: What procedure do you follow at Hanford for the determination of the extent of plutonium exposure following an accident?

Answer: We essentially follow the procedure which Dr. Foreman w1ll outline, i.e., early urine samples and rapid processing of these samples. Early urine analyses present some difficulty in interpretation. After $\frac{1}{2}$ to 1 day urinary excretion of plutonium follows a power function, but prior to this time the function is discontinuous depending on the nature of the individual. The results will depend on such factors as the quantity of urine in the bladder at exposure time and the time interval before sample collection. We attempted to determine the effects of some of these factors using pigs, but, unfortunately, it is difficult to get pigs to do what you want them to do when you want them to do it. The problem proved to be much the same with humans. Early blood samples offer a theoretical advantage at early times and probably would indicate a more rapid rise in plutonium levels than one would obtain from urine assay.

Dr. Foreman will discuss plutonium contaminated wounds. You will be interested to know that $W$. C. Roesch at Hanford has developed a method for measuring the amount of plutonium in wounds. Essentially, he determines the amount of plutonium beneath the skin in a wound by measuring the $17 \mathrm{kv}$ x-ray from plutonium using a thin sodium iodide crystal about $\frac{1}{2}$ in. in diameter. He estimates that the sensitivity of this technique is on the order of 2 to $4 \times 10^{-4} \mu \mathrm{c}$ of plutonium in a wound.

Harry Foreman, Los Alamos Scientific Laboratory

Probably the simplest way to discuss the medical management of a plutonium-exposed individual is to go through the course of events following an exposure and discuss the problems in the order in which they arise. 
It is of interest to point out that while contamination accidents are not infrequent, the situations which require medical care are exceedingly few. Of individuals at Los Alamos who were examined because of incidents involving surface contamination, far fewer than 1 per cent ever accumulated an appreciable body burden of plutonium as manifest by urinalysis.

Plutonium contamination accidents have occurred in a large variety of ways. Several resulted from the breaking of glass containers of plutonium or from the spilling, splattering, or spraying of plutonium solutions. Less frequent causes of contamination have been dry-box fires, inadvertent opening of containers of plutonium metal which had oxidized to fine metal-oxide dust, and undetected plutonium line leaks. On a number of occasions the time and nature of the contaminating event or events were never known and the contamination was picked up only through routine urine assays.

The immediate problem following an accident involving plutonium, obviously, is to remove the individual from the source of contamination. When an accident involves severe injury, the contamination problem is complicated by immediate first-aid needs. In this case it may be necessary to give treatment on the spot. The decision about this is a difficult one, but in general one might expect that only emergency life-saving procedures would be carried out in a contaminated area.

Once the individual is away from the contaminated area, the problem arises as to the suitable place to carry out surface decontamination and treatment. It is obvious one must avoid contamination of emergency rooms or any hospital room and equipment used by the public. To meet this need, a number of laboratories, e.g., Los Alamos, Livermore, and Argonne, have installed special treatment rooms designed to prevent spread of contamination. The design and use of these have been described by Finkel and Hathaway. ${ }^{1}$

The next decision of importance is whether to attempt surface decontamination or to do treatment procedures such as suturing and excisions first. Except for emergency procedures (checking of bleeding, removal of painful foreign bodies, etc.) it is probably desirable to remove the readily removable activity, first. Washing skin surfaces with a detergent solution containing about 10 per cent $\mathrm{Na}$ EDTA (ethylenediaminetetraacetate) or $\mathrm{Fe}-3$ specific* has been found efficacious. In all probability there are no deleterious effects from alpha activity on the skin since the alpha particles do not penetrate the cornified layer. For this reason it might be permissible to neglect tenaciously adhering activity on the skin rather than produce

*A chelating agent manufactured by the Versene Division of the Dow Chemical Company. 
abrasion by scrubbing or maceration through which plutonium might penetrate. It has been suggested that plutonium may deposit in hair follicles and affect the sweat and sebaceous glands. At present there is no evidence for this. The decision arises as to how rigorously one should pursue the removal of plutonium from mucous membranes or from the cornea. Here again, information on the degree of damage done by plutonium to such surfaces is lacking. However, common sense dictates that care should be taken not to produce breaks in the mucous surfaces.

After the decontamination is carried out and necessary immediate procedures done, attention can be turned towards more definitive types of treatment, i.e., those dealing directly with the plutonium that has gained entrance into the body. First to be considered is the question of excising contaminated or potentially contaminated wounds. In view of the difficulty of determining the actual amount of plutonium in a wound and the extreme hazard of minute quantities of plutonium, one is tempted to excise all potentially contaminated wounds, particularly if the excision is not a mutilating or disabling one. One can be guided to a certain extent in his decision by the surface activity on the skin about the wound and by the level of activity on the wounding object. In the early days of Los Alamos it was the practice to excise most of the potentially contaminated wound areas. In very few instances was an appreciable amount of plutonium removed. Recently, however, in one case $850,000 \mathrm{~d} / \mathrm{m}$ was removed on excision and $30,000 \mathrm{~d} / \mathrm{m}$ on a second excision. Findings of this magnitude readily lead one to feel justified in excising practically all uncomplicated potentially contaminated wounds. Fortunately, the excision of a wound area is not an immediate emergency procedure. One can delay the decision pending the accumulation of information. Hamilton ${ }^{2}$ has shown in his studies on rats that intramuscular plutonium largely remains fixed at the injection site. Ninety per cent of an injected dose was still remaining at the site after four days. Studies at Los Alamos verified this.

The next step is to estimate the amount of plutonium which might have gained entrance into the body. Since at least $24 \mathrm{hr}$ are required before reliable assay is available on the excreta, and since it is desirable to start treatment for hastening the excretion of the plutonium as soon as possible after the exposure, it is necessary to make an estimate of the body burden from the conditions of the exposure and from spot urine samples.

If it has been estimated that an appreciable body burden of plutonium has been accumulated, there are two procedures which might be used to hasten excretion of the metal. One is the zirconium citrate or zirconium malate procedure devised by Jack Shubert ${ }^{3}$ and the other is the Ca EDTA procedure. 4 won't go into the details of the mechanisms involved, but I'd like to discuss briefly the rationale for the choice of one over the other. 
If zirconium salts are given early, bone deposition is prevented and plutonium excretion is accelerated. This has been conclusively demonstrated in animals. However, I would hesitate to use it because of its side-effects. Two cases of zirconium malate administration for the removal of plutonium have been reported in England. In both cases the patients developed a very severe labrynthitis with severe vertigo which lasted for about one year. I might also mention that zirconium citrate is inconvenient to use since it can't be stored. The zirconium complex breaks down on autoclaving and deteriorates rapidly.

In view of the English reports and other evidence I feel that Ca EDTA is the medication of choice in hastening the excretion of plutonium. Calcium EDTA has been demonstrated to have nephrotoxic potentialities when given repeatedly and in large doses; $;^{5}$ however, when given in the recommended dose range and with properly interspersed rest periods, it is a safe drug to use. The individual receiving the drug should be followed closely with daily urinalysis and the drug stopped if casts or red cells appear.

The following are several case reports illustrating the dosage schedule, the method of determining the body burden, and the method of evaluating the effectiveness of the treatment of the drug.

\section{Case 1}

The first case concerns a 40-year old woman technician who, at the time of the accident, was working with a plutonium solution in $1 \mathrm{~N} \mathrm{HNO}_{3}$ that assayed approximately $2 \times 10^{6} \mathrm{~d} / \mathrm{m} / \mathrm{cc}$. The flask broke and a jagged edge of the broken contaminated glass cut her across the base of the thumb through a rubber glove. First-aid treatment consisted of cleansing the wound area with soap and water and stopping the profuse bleeding with a couple of sutures and a small pressure dressing.

From the conditions of the accident it did not seem likely that any appreciable activity had entered the wound. However, an alpha meter passed over the surface of her hand indicated a few hot spots as high as $1000 \mathrm{c} / \mathrm{m}$ after the initial cleansing. It was decided to collect urine in an attempt to learn how much, if any, plutonium had gained entrance into the body. The first day's collection of urine assayed $90 \mathrm{~d} / \mathrm{m}$. There was a possibility that this sample was contaminated, as occurs not infrequently in the first urine samples collected after an accident. However, subsequent collections on the following days showed continued excretion of measurable amounts of plutonium. It was apparent that a small amount of plutonium had been absorbed from the wound; therefore, it was decided to give her Ca EDTA. 
Before starting the treatment, the wound area was excised. The excised specimen assayed $1500 \mathrm{~d} / \mathrm{m}$, a very small amount. Calcium EDTA was given in $2 \frac{1}{2}-\mathrm{g}$ doses in $500 \mathrm{cc}$ of saline by slow intravenous drip twice a day for 4 days, stopped for 2 days, and then given again under the same regimen for 12 days. The first day of treatment brought forth an unusually high excretion; the count jumped from a pretreatment level of 12 up to 1380. As seen in Fig. 1, on subsequent days under treatment, the output continued high. When treatment was stopped, the level dropped sharply and rose again with renewed treatment. formula: ${ }^{6}$

Calculation of the body burden was made by the use of Langham's

$$
\mathrm{D}_{\mathrm{r}}=435 \mathrm{Ut} \mathrm{t}^{0,76}
$$

where $D_{r}$ is the body burden in $d / m, U$ is the urine excretion in $d / m$ on day $t$, and $t$ is the time in days after the exposure. Forty days after the accident the urine excretion level averaged $1.95 \mathrm{~d} / \mathrm{m}$ of plutonium per $24 \mathrm{hr}$. At this excretion level the equation yields a body burden of $14,000 \mathrm{~d} / \mathrm{m}$.

As seen from Langham's data, under ordinary conditions the body burden of plutonium at 30 days is 96.3 per cent of an initial acute dose, which in this case would give $14,000 / 96.3=14,540 \mathrm{~d} / \mathrm{m}$. To obtain the actual initial dose, the amount excreted under treatment must be added to this value, giving $14,540+4500=19,040 \mathrm{~d} / \mathrm{m}$. With an initial value of this magnitude the body burden at 30 days without treatment would have been $19,040-.037(19,040)$ or $18,350 \mathrm{~d} / \mathrm{m}$. The actual body burden was $14,000 / 18,350$, or approximately 76 per cent of this. The difference of 24 per cent is an index of the effectiveness of the treatment. It is likely that had the drug been started almost immediately after the accident, instead of 5 days later, the treatment would have been even more efficacious.

\section{Case 2}

The next case was a more complicated one. The individual involved had been working with plutonium in various forms for a number of years and had had several minor contamination accidents. He was slowly accumulating plutonium internally as evidenced by a gradually rising urine count over the years. It was just about decided to remove him from his job and place him on work away from plutonium when he had this rather serious accident.

The accident occurred while he was working in a dry-box used for processing plutonium residues. He was placing rubber tubing over the outlet of a suction flask when the tip broke and he was cut across the ball of 


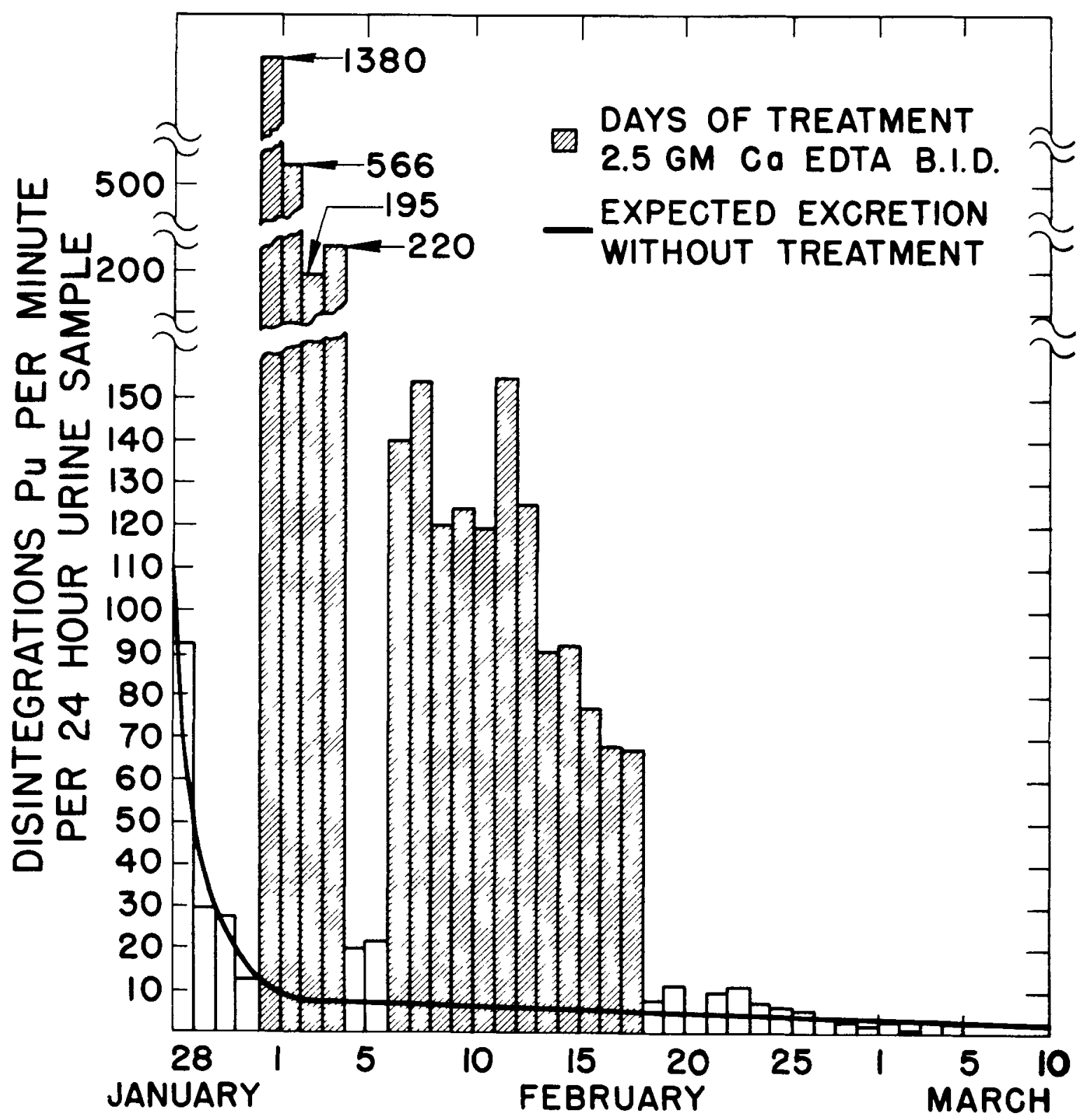

Fig. 1 Urinary excretion of plutonium following Ca EDTA treatment, Case 2. 
the thumb. The flask was highly contaminated with plutonium in various forms; the nitrate, fluoride, oxide, etc. Surface counts of both the glove he was wearing and the skin about the wound were off scale on the alpha meter.

He was taken to the hospital, where the wound area was immediately excised. Analysis of the tissue yielded $850,000 \mathrm{~d} / \mathrm{m}$. The removal of this tremendous amount amply justifies the practice of excising all potentially contaminated wounds.

Treatment with Ca EDTA was started about one hour after the accident. Results of the urine assays are shown in Fig. 2. The urinary excretion of plutonium for the first day (from about 4:30 in the afternoon, when the accident occurred, until the following morning) was $80 \mathrm{~d} / \mathrm{m}$. It was expected from the conditions of the accident that there would be a tremendous excretion, but, surprisingly enough, the value was low. Then, instead of dropping as had happened in previous cases, the excretion rose on subsequent days. This rising level was interpreted to mean there was still plutonium left at the wound site that was slowly feeding into the blood stream.

After 4 days treatment was stopped. The reasoning was as follows. Only a little over $1000 \mathrm{~d} / \mathrm{m}$ had been put out in the 4 days. If the treatment were very effective, i.e., this excretion level represented a high percentage of the patient's body burden, then he must not have had much plutonium in him. The other alternative was that the low level of excretion was due to the fact that the treatment was not being very effective. In either case it was advisable to stop treatment. With the stopping of the drug, the urinary plutonium excretion dropped to $27 \mathrm{~d} / \mathrm{m} /$ day and stayed about this level for 5 days. This gave further confirmation to the impression than an intramuscular source was feeding into the blood stream since the excretion level would have been expected to drop sharply at this time had a single acute exposure occurred. Another 2 days of Ca EDTA treatment was given and again the excretion went up, this time even higher than on the fourth day. It was decided to excise more of the wound area. The second tissue piece assayed $30,000 \mathrm{~d} / \mathrm{m}$. The effect of the excision was immediately manifest in the urine. The excretion began to fall and dropped steadily to a level of $9 \mathrm{~d} / \mathrm{m} /$ day about one week after the second excision. One year after the accident, the level had fallen to approximately $4 \mathrm{~d} / \mathrm{m} / \mathrm{day}$.

At the time the excretion level was on the order of $9 \mathrm{~d} / \mathrm{m} /$ day, it was possible to do further studies to gain more information about Ca EDTA and plutonium excretion. In one such study a comparison between orally administered and intravenously administered Ca EDTA was made. A 3-day trial of $2 \mathrm{~g}$ a day of $\mathrm{Ca}$ EDTA was made for each mode of administration. The oral treatment produced no change in excretion above the control levels. Intravenous administration resulted in a tenfold increase in urinary plutonium 


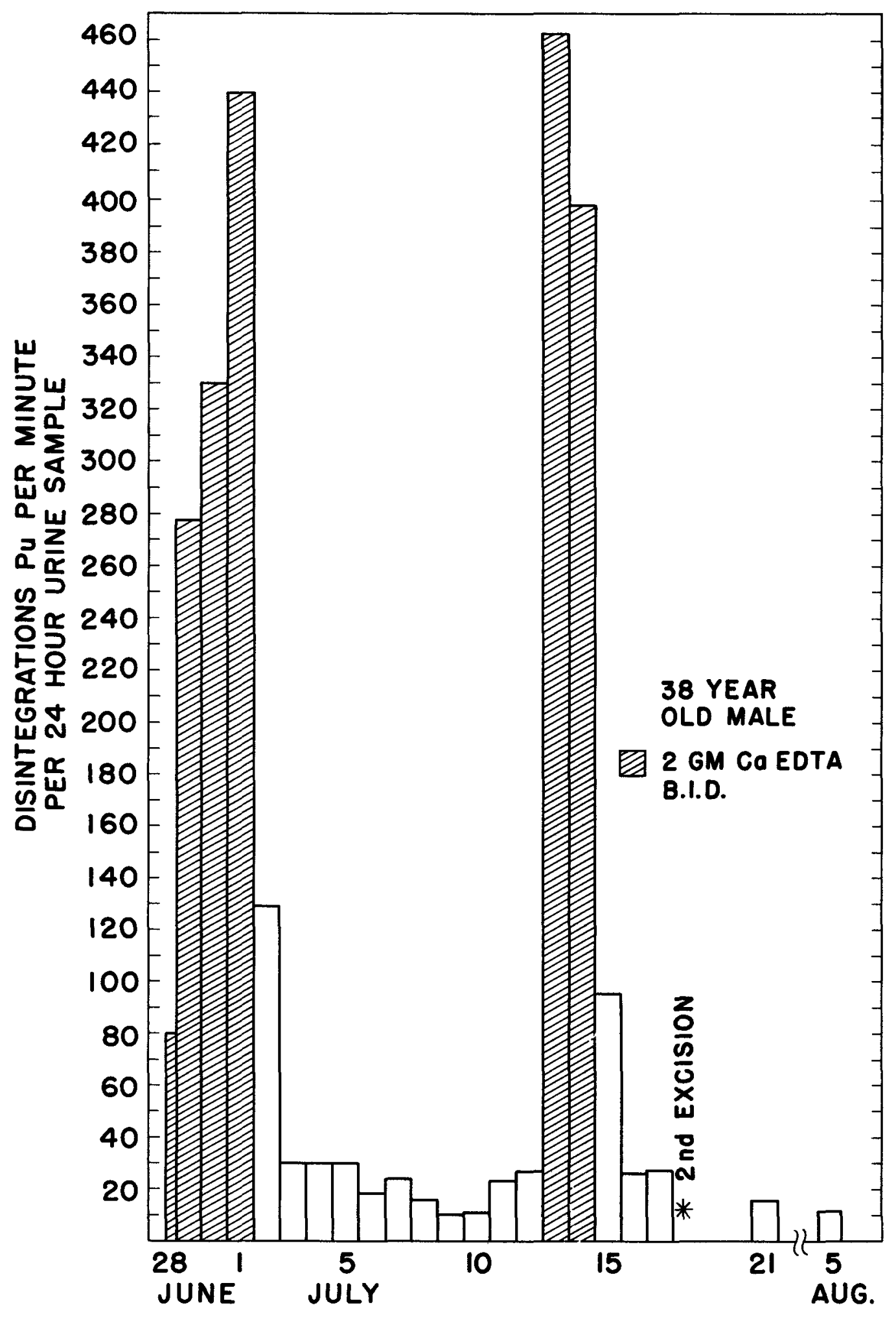

Fig. 2 Urinary excretion of plutonium, Case 2. 
excretion. Fecal excretion assays were also made at these times. The feces : urine excretion was approximately $1: 1$ in the control period and did not change during the oral-treatment period. Intravenous administration of the drug did not alter the fecal excretion.

The net increase in excretion brought about by the Ca EDTA was not significant. The administration of the drug was of value primarily because of the diagnostic information it gave, namely, in pointing out the residual intramuscular deposit. The apparent ineffectiveness of Ca EDTA in this case might well have been because of the very low blood levels of plutonium present at any one time, i.e., the small amount of plutonium that was being fed slowly from the intramuscular site into the circulation.

Using the mode of calculation described above, it is estimated that the body burden at 70 days after the accident was approximately $50,000 \mathrm{~d} / \mathrm{m}$.

Case 3

The following is an americium exposure case wherein Ca EDTA was particularly effective. This case involved a skilled radiochemist who, at the time of the accident, was working with a very "hot" mixture of $\mathrm{Am}^{241}$, $\mathrm{Am}^{242}, \mathrm{Cm}^{242}$, and associated fission products contained in a quartz ampoule. A gamma-ray meter held 6 in. from the ampoule read $150 \mathrm{r} / \mathrm{hr}$. This mixture had been obtained by irradiating $5 \mathrm{mg}$ of $\mathrm{Am}^{241}$ in a high-neutronflux pile. Just as the ampoule was about to be opened, it exploded (for reasons still undetermined), forcing the contents over the top of the lead shield in front of the working space and spraying his face and hair. Almost immediately he scrubbed thoroughly with soap and water, but even after thorough washing there remained "hot" spots over his forehead, cheek, and upper lip that went off scale on the alpha meter and that read over $20 \mathrm{mr} / \mathrm{hr}$ on the beta-gamma meter. He was taken to the hospital, where physical examination revealed only minor abrasions over his forehead and in the mucous membranes of his mouth and nares.

The first urine sample, obtained approximately three hours after the accident, assayed 2 to $3 \mathrm{~d} / \mathrm{m} / \mathrm{cc}$. The next urine sample, $1 \frac{1}{2}-\mathrm{hr}$ later, showed an even higher count. This rising count strongly indicated that he had accumulated a body burden of radioactivity, probably through the abraded areas on his skin and mucous membranes and possibly by inhalation. Undoubtedly, some material was swallowed since gastric washings assayed over $1000 \mathrm{~d} / \mathrm{m} / \mathrm{cc}$.

It was decided to attempt therapy, i.e., to use a means to accelerate the excretion of the radioactivity. Calcium EDTA was administered intravenously in $2-\mathrm{g}$ doses in $250 \mathrm{cc}$ saline twice a day for 5 days, starting 
$8 \mathrm{hr}$ after the accident. A blood sample drawn at the time treatment was started assayed $3 \mathrm{~d} / \mathrm{m} / \mathrm{cc}$. For the first 2 days the patient was hospitalized for observation and to assure full collection of urine and feces. He was ambulatory at all times and was able to help with the monitoring and to take full precautions to prevent contamination of hospital equipment. During his hospital stay the remaining "hot" spots on his skin and hair were removed by repeated scrubbing with a mixture of detergent and Ca EDTA solution. Shortly after admission to the hospital, a series of laboratory tests were run to serve as a base line against which to compare changes which might result from the radiation exposure or possibly from the use of the drug. These tests included a CBC with a platelet count, complete urinalysis, serum proteins, serum calcium and phosphorous, serum alkaline phosphates, and prothrombin time. Urinalysis was repeated daily while treatment was going on. Hematological tests and serum chemistry were repeated in a week and after a month. No significant changes were ever noted.

After leaving the hospital, treatment was continued at the same dosage level for 3 more days on a come-and-go basis in the first-aid room. Following that, Ca EDTA was given only as a diagnostic aid at times indicated in Fig. 3.

The results of urine excretion assays are presented in Fig. 3, expressed as disintegrations of americium* per minute per $24 \mathrm{hr}(\mathrm{d} / \mathrm{m} / 24 \mathrm{hr})$. During the first $24 \mathrm{hr}$ urine excretion was about $11,000 \mathrm{~d} / \mathrm{m}$, a surprisingly high amount, indicating either a high degree of effectiveness for the treatment or the presence of a dangerously high level of activity in the body. Fortunately, the former turned out to be true. The excretion level remained high in the first few days and dropped abruptly with cessation of treatment, rising again sharply when treatment was reinstituted. One month after the accident urine excretion levels had fallen to approximately $0.5 \mathrm{~d} / \mathrm{m} / 24 \mathrm{hr}$, the lowest level of significant detection by the analytical procedure used. Urine samples were assayed monthly from then on. The assays ranged from 0.12 to $0.30 \mathrm{~d} / \mathrm{m} / 24 \mathrm{hr}$, values which would not be taken to indicate with any certainty the presence of americium internally. One could not distinguish these urine samples from others obtained from individuals who had had no americium exposure.

* Because of the short half-lives of most of the isotopes in the contamination mixture, the only appreciable activity detectable in a few days after the accident was that associated with $\mathrm{Am}^{241}$ and $\mathrm{Cm}^{242}$. Pulse-height analysis of the activity in the urine showed the $\mathrm{Am}: \mathrm{Cm}$ ratio to be $16: 1$. Since the hazard from $\mathrm{Cm}$ is almost the same as that from Am, no great error was introduced by considering all of the activity to be Am. 


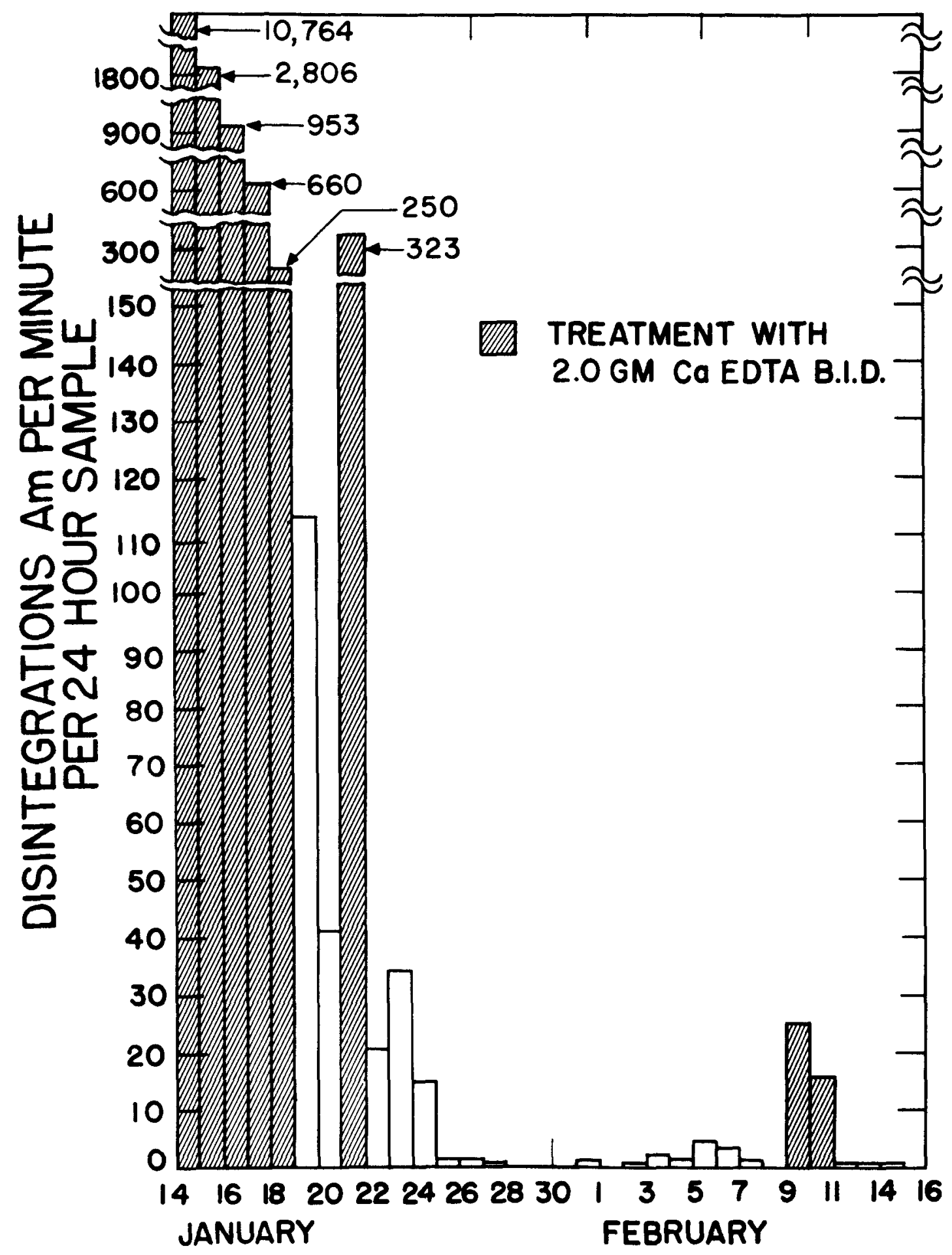

Fig. 3 Urinary excretion of americium following Ca EDTA treatment, Case 3. 
Approximately one year after the accident an additional trial of $\mathrm{Ca}$ EDTA was given. The urine excretion level rose from a pretreatment level of 0.2 to $4.2 \mathrm{~d} / \mathrm{m}$ on the first day of treatment, to 2.4 and $1.6 \mathrm{~d} / \mathrm{m}$ on subsequent treatment days, and dropped to $0.3 \mathrm{~d} / \mathrm{m}$ the day after treatment was stopped. Calcium EDTA, used in this fashion, served as a diagnostic aid to demonstrate the presence of a body burden of activity not otherwise detectable.

Fecal samples were collected for the first 6 days. The results of assay of these samples are given in Table 1. The very high fecal excretion in the 6 days, over $80,000 \mathrm{~d} / \mathrm{m}$ of americium, was undoubtedly in a large measure due to material that was swallowed and excreted, rather than material which had actually gained entrance into the circulation and was being excreted through the bowel.

Table 1

FECES ASSAY FOR AMERICIUM EXPOSURE IN CASE 3

$\begin{array}{crr} & & \begin{array}{c}\mathrm{d} / \mathrm{m} \text { Am } \\ \text { per } 24-\mathrm{hr} \\ \text { Specimen }\end{array} \\ \text { January } & 14 & 14,800 \\ 15 & 41,200 \\ 16 & 19,000 \\ 17 & 1,541 \\ 18 & 2,105 \\ 19 & 1,710 \\ 20 & 438\end{array}$

With the data at hand it was possible to estimate the present body burden, the effectiveness of the treatment, and the initial dose taken in. The calculation of the body burden was made by using an empirical excretion curve obtained from humans for plutonium and extrapolating to americium by use of a factor. The assumption was made that the treatment did not alter the slope of the excretion curve at times long after the treatment was stopped. The equation of the excretion curve for plutonium is Langham's formula as given in Eq. 1, where $D_{r}$ is the body burden in $d / m, U$ is the 
urine excretion in $\mathrm{d} / \mathrm{m}$ on day $\mathrm{t}$, and $\mathrm{t}$ is the time in days after the exposure. The factor for adapting the equation for use with americium was obtained from Carter' $\mathrm{s}^{7}$ study in animals, wherein it was shown that plutonium and americium urine excretion curves are approximately parallel and that at a given time, i.e., 30 days, after the entrance of the isotope into the body, the urine excretion of americium is twice that of plutonium. With this information adapting the equation for use in americium cases was simple, namely:

$$
\text { D. } \left.(\text { americium })=1 / 2 \mathrm{D}_{\mathrm{r}} \text { (plutonium }\right)=1 / 2\left(435 \mathrm{Ut} \mathrm{t}^{0.76}\right) \text {. }
$$

In the case under discussion, at 30 days, the urine americium excretion, $U$, was $0.45 \mathrm{~d} / \mathrm{m}$. Putting this datum into the equation gave a body burden of $1350 \mathrm{~d} / \mathrm{m}$ at 30 days.

The calculation of the amount of activity entering the body was made as follows. Langham (again from human plutonium studies) found the amount of plutonium remaining in the body at 30 days after acute exposure is 96.3 per cent of the initial dose. For americium, using the same factor as above, this would be approximately 92.5 per cent. With a body burden of $1350 \mathrm{~d} / \mathrm{m}$ the corresponding initial dose is 1460 , or approximately 1500 . However, in the present case, treatment resulted in the excretion of an additional 16,000 (this neglects the approximately $150 \mathrm{~d} / \mathrm{m}$ which would have been excreted normally but this is negligible compared to the 16,000 ), so the actual initial dose must have been $1500+16,000$ or approximately $17,500 \mathrm{~d} / \mathrm{m}$.

The efficacy of the treatment can be judged by comparing the present body burden with that which would have resulted had no treatment been given. With an initial dose of $17,500 \mathrm{~d} / \mathrm{m}$ the amount remaining in the body at 30 days (without treatment) would be the initial dose $(17,500)$ minus the amount excreted $(0.075 \times 17,500)$, namely, $16,000 \mathrm{~d} / \mathrm{m}$. This value compared to 1350 indicated that the treatment was decidedly efficacious. As a result of the treatment the body burden is less than one-tenth of what it would have been had treatment not been instituted.

\section{References}

1. Finkel, A. J., and Hathaway, E. A., "Medical Care of Wounds Contaminated with Radioactive Materials," J. Am. Med. Assoc., 161, 121-126 (1956).

2. Scott, K. G., Axelrod, D. J., Fisher, H., Crowley, J. F., and Hamilton, J. G., "The Metabolism of Plutonium in Rats following Intramuscular Injection," J. Biol. Chem., 176, 283-293 (1948). 
3. Schubert, J., "An Experimental Study of the Effect of Zirconium and Sodium Citrate Treatment on the Metabolism of Plutonium and Radioyttrium," J. Lab. Clin. Med., 34, 313-325 (1949).

4. Foreman, H., "The Use of Chelating Agents for Accelerating Excretion of Radioelements," J. Am. Pharm. Assoc., 42, 629-632 (1953).

5. Foreman, H., Finnegan, C., and Lushbaugh, C. C., "Nephrotoxic Hazard from Uncontrolled Edathamil Calcium-Disodium Therapy," J. Am. Med. Assoc., 160, 1042-1046 (1956).

6. Langham, W. H., "The Application of Excretion Analyses to the Determination of Body Burden of Radioactive Isotopes," Brit. J. Radiol., Supplement No. $\underline{7}, 95-113$ (1956).

7. Carter, R. E., and Langham, W. H., "The Relative Physiological and Toxicological Properties of Americium and Plutonium," Los Alamos Scientific Laboratory Report LA-1309, Nov. 15, 1951.

S. M. Sanders, Jr., Savannah River Plant*

The procedure used at the Savannah River Plant for the determination of urinary plutonium was described at the October, 1955, Blo-Assay and Analytical Chemistry Meeting held at Fernald and later published as an unclassified AEC Research and Development Report, DP-146. This procedure is still used routinely by the Savannah River Bio-Assay Laboratory, but at the same time a program for improvement of the procedure is being carried out.

The program is divided into an immediate and a long-term phase. The immediate phase is devoted to shortening the present procedure and increasing the sensitivity; either of these improvements would make the analyses less expensive and less time-consuming. A reduction in the large number of manipulations required for each analysis would increase the limited capacity of the laboratory and, consequently, the coverage of the blo-assay program. Any increase in the sensitivity would reduce the volume of urine necessary for analysis, and this would reduce the time needed to obtain a sample. The

* This talk concerns development work pursued at the Savannah River Plant and does not imply the adoption of the techniques for routine procedure. 
long-term phase of the program is devoted to finding a new and better procedure for plutonium analyses.

The present procedure, used routinely, includes two bismuth phosphate coprecipitations, two lanthanum fluoride coprecipitations, a thenoyltrifluoroacetone (TTA) solvent extraction, an electrodeposition, and an autoradiographic determination. This procedure, like that used at Hanford, electroplates the plutonium on an area $7 \mathrm{~mm}$ in diameter.

The revisions which we have effected in the procedure and may use routinely in the future will be described. Since the bio-assay group prefers not to evaporate the urine, a single bismuth phosphate coprecipitation is used to separate the plutonium from the urine. After $350 \mathrm{mg}$ of bismuth have been precipitated slowly from the urine as the phosphate and allowed to digest, the supernatant is aspirated and the precipitate filtered and muffled. Muffling the precipitate eliminates the need for a second bismuth phosphate coprecipitation and treatment with perchloric acid to remove the organic matter. Two lanthanum fluoride coprecipitations are still found necessary prior to solvent extraction.

Solvent extraction in the revised procedure is very similar to that presently used at the Savannah River Plant and at Hanford. Two, rather than $40 \mathrm{ml}$, of aluminum nitrate solution is used to dissolve the $1-\mathrm{mg}$ lanthanum fluoride precipitate, no sodium nitrite is added prior to extraction, and only $2 \mathrm{ml}$ of TTA solution is used for the extraction. The salts present in the electrolyte are reduced by using $5 \mathrm{ml}$ of $3 \mathrm{~N}$ hydrochloric acid, in two portions, to back-extract the plutonium from the organic phase. Previously $15 \mathrm{ml}$ of $8 \mathrm{~N}$ hydrochloric acid had been used. We were able to reduce the amount of acid by adding $10 \mathrm{ml}$ of toluene to $2 \mathrm{ml}$ of TTA solvent. The electrolyte is heated before electrodeposition to drive off any toluene which may be held by the aqueous phase. Residual toluene will attack the Lucite plating cells.

The electrodeposition step is much the same as that previously described except that we have reduced the plating area. We had been electroplating the plutonium on an area $7 \mathrm{~mm}$ in diameter, but the device used to scan the autoradiographic emulsions scans only one-third of this area. The sensitivity of the procedure was tripled by reducing the plated area to 149 mils $(3.785 \mathrm{~mm})$ in diameter. This reduction has enabled us to reduce the amount of urine collected by one-third.

Urine collection has been a problem at Savannah River. We have approximated a 24-hr sample by collecting $1500 \mathrm{ml}$ of urine representing several voidings of a single individual on the job. The employee must change from work clothes to decontaminated clothing and walk some distance to a clean change room to void. The sample collection has taken as long as 2 to 3 weeks, and by reducing the amount to $500 \mathrm{ml}$, we hope to shorten 
collection time to 2 or 3 days which will permit us to detect unknown exposures much sooner.

Electrodeposition on the 149-mil area requires more work to establish conditions for optimum recovery. Good recoveries have been obtained using the Hanford ammonium nitrate-nitric acid electrolyte. However, reagent concentration and $\mathrm{pH}$ must be too carefully controlled for routine analyses by technicians. We have gone back to the Hanford potassium hydroxide solution but this does not give completely reproducible results with the small plates. The search for an ideal electrolyte is being continued.

Replacement of the Lucite plating cells with Teflon has been considered. When Teflon is used, the electrolyte is made with 10 per cent methanol to reduce the surface tension of the aqueous solution and allow complete wetting of the Teflon. Plating on the polished end of a stainless steel wire also has been tried. The stainless steel wire was used as the stirring anode. This technique was successful, but it is too difficult to make electrodes for each analysis.

The autoradiographs have been improved by the addition of a trackeradication step prior to exposure. The emulsion slides are treated as follows: (1) 3-min agitation in a formalin-sodium-carbonate hardening solution, (2) 2-min wash in water, (3) 10-min agitation in dilute chromic acid solution, (4) 8-min wash to remove the chromic acid. The film is then airdried and exposed for the usual 1-week period, which has not been shortened. With the increased sensitivity of the method we had the choice of reducing urine volume and collection time or the exposure time. We felt that more was to be gained by reducing the collection time. The emulsion development procedure is unchanged except for the substitution of a rapld actd-fix solution containing ammonium thiosulfate.

Our instrument group has developed an alpha scintillation counter with a background of $0.01 \mathrm{c} / \mathrm{m}$. The tube is arranged so that the source is brought right up to the small phosphor. The tube and the other components of the counter have been chosen to reduce background noise. The source must be plated on a $\frac{1}{2}$-in. disk for use with this counter. We will use this counter in the event of accidents or other cases where results must be obtained quickly.

Our improved procedure is expected to reduce the time necessary for sample collection from 14 to 3 days and the analysis time from 4 to 2 or $2 \frac{1}{2}$ days.

We have done work on other methods for separating plutonium from urine. Attempts to coprecipitate plutonium from urine by adding egg albumen and then denaturing the albumen gave very poor recoveries. Millipore filter paper was used in the coprecipitation step and was found to be too slow. Attempts to separate nonmetabolized plutonium from urine with 
a Dowex -50 resin column gave poor results. Plutonium has been successfully separated from 6 to $8 \mathrm{~N}$ acid solution with an anion exchange column. These recoveries have been promising, and this technique may prove useful for isolation of plutonium after preliminary separations. 


\section{THE FISSION-PRODUCT PROBLEM}

Payne S. Harris

Los Alamos Scientific Laboratory

University of California

Los Alamos, New Mexico

I have been asked to give a "philosophical" talk about the fissionproduct problem. I am going to confine my discussion to the general problem since other papers will deal with some of the more concrete problems connected with fission products.

The most common source of fission products is, of course, weapons. Fission products have been with us since we started firing nuclear weapons, but they did not come to the attention of the lay public until the Castle Test Series of 1954 at Eniwetok and Bikini Atolls. However, most of us, particularly those of us who have been involved in the various tests, have been concerned about fission products for a long time. Weapons produce fission products, and substantial quantities are usually released by a large-yleld (thermonuclear) device.

It was announced, after the latest overseas test series, that a so-called "clean" thermonuclear device had been shot. With this type of system, fission products from weapons therefore may possibly be relegated to the insignificant position which they had before the advent of a large-scale thermonuclear device, that is, insignificant from the lay point of view. We shall still have the fission-product problem in the immediate test areas. Others will continue to study the weapons fission-product problem from the longrange viewpoint. The Army Medical Service Graduate School at Walter Reed Hospital has been studying the uptake of fission products by individuals living at great distances from the test site, from bombs of small yield which were shot in Nevada. This is being done with excretion studies which have correlated very well with the time of cloud passage. A measurable uptake of certain fission products by individuals has been found, and Dr. Schrodt will 
report on this later in the program.

The other source of fission products is reactors of all types. Those who have been connected with the use of reactors for production, development, and research are well acquainted with the problems involved here. I'd like to give an example or two of how the fission-product problem may become, effectively, world-wide even if we consider only reactors as the source.

There are today on the drawing boards and under construction nuclearpowered aircraft. It has been suggested by the Air Force that we may have several of these aircraft in the air continuously, that is, $24 \mathrm{hr}$ per day, necessarily operating at a generous power level. Depending on design, various proportions of the total fission products will be released in the air during operation of the alrcraft. If you run several large reactors in the air $24 \mathrm{hr}$ a day for one year, the total amount of fission-product production per year is equivalent to many megatons of energy released by a nuclear explosion. If one of these aircraft, after operating for one year, crashed and the reactor caught fire and burned, there would be released in the crash area fission products equivalent in amount to the total fission products produced during the entire running time of that reactor. For example, one aircraft with one-year's operating time on the reactor would put out the equivalent of a few megatons of $\mathrm{Sr}^{90}$.

Another reactor development which has been announced is the use of nuclear power for rocket engines. According to one plan, the nuclearpowered engine would not be used until the third stage, where the rocket itself would be outside of our atmosphere. These rocket engines must be tested however, and they will expend a fair amount of energy. It is possible during testing that we will have ruptures of the canned units in the engines. If this material were put out at present testing rates, a few kilotons of energy per day of operating time could be released into the atmosphere. Again, some of the limits on the use of power reactors are going to be the production of fission products in the reactor, how to get rid of them, and where to bury them. At the present time reactors are controlled and are not continually loosing their total fission-product production into the atmosphere. However, it seems certain that with the advent of new reactors for new uses, it will become more economical under operating conditions to release at least certain fractions of the total fission products produced. So, even though we may build absolutely clean thermonuclear weapons, or even if we stop the testing of all types of nuclear weapons, the fission-product problem is still going to be with us. And as more production of nuclear power for general use evolves, the problem will not remain restricted to the small groups operating under the aegis of the AEC as it is now. It will become more widespread, and I certainly believe that it may become, from the standpoint of power alone, a world-wide proposition. 
The second point that I should like to discuss is fission-product distribution. Distribution can be subdivided into local and world-wide. Returning to weapons, if we talk about small atomic devices with yields of less than $200 \mathrm{kt}$, the contamination problem from fission products produced is essentially a local one. Similarly, the contamination problem from present reactor uses is essentially local. Here at Los Alamos with our reactors, which operate at very low power, it has been a very small problem. This is also true at Hanford, where a detailed study of local environmental contamination has been under way for some time. World-wide contamination becomes extremely important from the testing of our present thermonuclear weapons and when one considers the future uses of atomic power. At present we can say that the majority of the individuals exposed to fission products are in a reasonably restricted area and in that sense are partially controlled. Plant personnel are controlled by the plant operators, and people who live in the plant area are under partial control, i.e., what we might call "exposure control, " by the management of the plant. When we consider worldwide contamination, either from bombs or from reactor operation and accidents, uncontrolled populations enter the picture. In the case of local contamination to which the controlled populations are exposed, the fissionproduct material enters the biosphere almost immediately. By this I mean that the fission products become avallable for intake without an interim transmission through some other medium. The transfer of flssion products from a small atomic bomb or from a reactor to the human may be in stages. For example, the contamination of surface and of water allows a single-stage transfer to the individual or a two-stage transfer with an animal or vegetable intermediate host. In the case of certain of the fission products the transfer is direct to the human via the lung.

With world-wide contamination the transfer from source to individual is much more complex. World-wide contamination from our present sources, thermonuclear weapons, is achieved by steps. The first step is the production of the material at the source with a distribution of 50 per cent of the available fission products within a few hundred square miles. The remainder of the material goes to the stratosphere, above the tropopause, where it is contained for a period of years and from which it slowly equilibrates with the biosphere at ground level and becomes available for intake by the human. Operation Gabriel, which has been carried on for some years by the AEC, has indicated that the mean life of transfer from the stratosphere to the troposphere, to the biosphere, and to the human is on the order of ten years. This means that if we stopped all testing and production of fission products from thermonuclear materials, we would reach an equilibrium level with the human some 17 years from now. There is a latency in the world-wide condition of contamination which one must recognize. The transfer to the human 
of the materials coming from the stratosphere into the biosphere is apparently not direct, i.e., it is not being taken into the individual directly through the lung. Some of the information which Dr. Anderson will present will indicate the hosts or intermediate stages. It appears that the fission products must go through a series of plant or animal steps before they become avallable to the human. Dr. Anderson has been making a series of measurements of the $\mathrm{Cs}^{137}$ uptake from world-wide contamination.

After considering briefly the source and distribution of fission products, I should like to discuss what I call "diagnostic probabilities." We are interested in finding out where the fission products are, the amount contained in individuals, and which ones we should look for. These are the underlying problems for the bio-assayer. There are two things to be considered in this picture: one is time and the other is human metabolism. In the case of the time parameter one has to consider the availability of any particular isotope within the fission-product spectrum. The isotopes prominent at early times following production are all the iodines, $\mathrm{Ba}^{140}, \mathrm{La}^{140}, \mathrm{Sr}^{80}, \mathrm{Mo}^{89}$, those from the noble gases $\mathrm{Xe}$ and $\mathrm{Kr}, \mathrm{Ce}^{141}$, and various others. At long times after production the prominent isotopes are $\mathrm{Sr}^{90}, \mathrm{Y}^{90}, \mathrm{Cs}^{137}, \mathrm{Ru}^{106}$, and $\mathrm{Rh}^{106}$. It is important to the bio-assayer to know when the isotope became available to the biosphere so that he may know which isotope or isotopes to look for in order to evaluate the general exposure. The isotopes prominent at later times are generally of lower specific activity and in lesser concentrations because of decay in the total mixture. One has to use a more sensitive method of assay, therefore, than one does for the early isotopes. If the material has entered directly into the individual with no intermediate steps, we look for isotopes of the first group. Later on, or if there are many intermediate steps between the production source and the human, we look for the second group.

The search is further restricted by human metabolism. There is at present a fair amount of information on this subject but most of it is extremely old. J. G. Hamilton's data on the feeding, inhalation, uptake, and excretion of single and mixed fission products are probably some of the best that we have. There has been insufficient work on these problems to give us better data. For example, $\mathrm{Cs}^{137}$ is given in NBS Handbook 52 with a turnover time of 15 days and an uptake of 100 per cent since it is an ionizable salt. These data were taken from Hamilton's studies which were done in the early days. Work in progress at Los Alamos, using the human counter, shows that the turnover time is greater than 100 days. In this case the Handbook is in error by something on the order of a factor of 10 . Much of the information which we have on uptake, metabolism, and excretion of fission products is at least 15 years old, and some of it may be in error by as much as the $\mathrm{Cs}^{137}$ figures. 
Transfer of fission products into the body medium is generally through the lung or via the GI tract. Transfer from the lung to the rest of the body depends in some cases on the chemistry of the isotope, and in others on the physical properties of the particle, the particle size, rate of solution in body fluids, etc. For instance, among the early isotopes iodines have an uptake in the body of 100 per cent whether they enter via the lung or the GI tract; however, only 10 per cent of the barium and strontium are absorbed from the GI tract and 10 per cent or more from the lung depending on the physical properties of the particles. Many of these factors controlling contamination in the individual have not as yet been accurately worked out. Similarly, the retention and excretory mechanisms are involved. For instance, if an intermediate-host transfer is needed, e.g., through a food animal into the human, intra-animal distribution and excretion from the animal play a part in the total amount that gets into the human. A material bound in bone with negligible distribution throughout the soft tissues could be concentrated to a great extent by a food animal but still be of no consequence in the human, unless one likes bones. A prominent early isotope is $\mathrm{Ce}^{141}$, which is apparently biologically unimportant. We were unable to find this isotope in the urines from Rongelap natives which we examined. This may however be due to the lack of a sensitive method for analysis rather than to a lack of cerium uptake and metabolism by the individual. So again, human metabolism restricts the number of isotopes of analytical interest.

The third limitation is the method of examination. If you are going to examine excreta, you are limited to those isotopes which are excreted in detectable quantities. If you are fortunate enough to have a human counter, you can look for isotopes that are gamma emitters, have a high uptake, and have a longer than instantaneous turnover time. Thus, the fission products of interest to the bio-assayer can be narrowed to a few major isotopes. I do not believe that fractionation occurs after production and the fractionation produced in the intermediate-host steps through plant or animal. In other words, I don't believe that it is necessary to look at the entire spectrum of fission products just because you don't know the fractionation absolutely. You can determine only a few of the total fission products and arrive at a good approximation of the contamination level. Ruthenium is a good example of this type of thing. In 1954 we went to Kwajalein and collected large quantities of urine from the Rongelap natives who had been transferred there after the March 1 detonation. We analyzed this urine to try to show that the internal hazard from fission products was, in this case, negligible compared to the external hazard. Dr. Bugher suggested that we look for ruthenium in these samples since the British had found that this element was concentrated in certain plants following tests in Australia. We looked 
for ruthenium and found it in extremely small quantities, which is not surprising since the uptake by humans is on the order of 0.001 per cent. The point I am making is that certain foodstuffs do concentrate ruthenium which is then ingested by the individual, but you have another filtration process in the human body so the ruthenium is not absorbed and retained by the human. Hence, the detection of ruthenium in excreta does not give you a criterion for the fission-product body burden. Another example is $\mathrm{Ce}^{141}$, which has a very small uptake. In the examination of excreta at early times the ideal isotopes are the iodines, $\mathrm{Ba}^{140}$, and $\mathrm{Sr}^{89}$ and at long times $\mathrm{Sr}^{90}$ and $\mathrm{Cs}^{137}$. With a human counter, where one is restricted to gamma emitters, at early times lodine is your best isotope and at later times $\mathrm{Cs}^{137}$. As an aside, it is of interest to note how little is really known about the history of iodine in the body. Physicians have been giving microcurie tracer doses of it for years. Therefore, the uptake is well known and the excretion is well known for the first $24 \mathrm{hr}$. Beyond that time it is hard to find anything in the literature on the subject. In the native urine we found that iodine was the most prominent early-time fission product. Chemical separation of iodine from human urine is a real problem, so we were fortunate in that we had counter systems which required little or no separation. We also examined the urine for the $\mathrm{Ba}-\mathrm{La}$ mixture, $\mathrm{Sr}^{89}$, the $\mathrm{Ru}-\mathrm{Rh}$ complex, and $\mathrm{Ce}^{141}$, which we did not find with our methods. We found that the iodines, $\mathrm{Ba}^{140}$, and $\mathrm{Sr}^{89}$ were the best isotopes to look for. This was true, not because of the amount produced or taken into the body, but because of the interposition of the human as the uptake-excretion path.

This brings up the problems which I would like to give to this audience. When we collected our native urine, we did not know which fission products to look for, nor in most cases did we have avallable procedures for the determination of the individual isotopes. I would like to see a detalled flow sheet set up for the examination of excreta for fission products at both early and late times. We have such a flow sheet for the examination of fllter papers which is, of course, a much simpler task than the analysis of urine. The other urgent problem, which I mentioned before, is the need for new experimental data on the actual uptake, internal metabolism, and excretion of the various fission products. 


\section{THE DETERMINATION OF IODINE-131 AND STRONTIUM-90 IN URINE}

Ariel G. Schrodt

Walter Reed Army Institute of Research

Washington, D. C.

Introduction

I would like to talk about some of the specific problems associated with fission products. The Biophysics Department at Walter Reed Army Institute of Research became interested in this problem in the winter of 1954 . We were originally interested in $\mathrm{I}^{131}$ in urine samples since iodine would be a considerable hazard in the case of an acute exposure - the type that troops in the field might have. We set up a program during the Teapot test series to examine urine samples for $\mathrm{I}^{131}$. The samples were collected from a number of stations throughout the world. Initially, we did not plan to examine the samples for $\mathrm{Sr}^{90}$ or any of the other fission products.

In order to determine the amount of iodine we might expect, we assumed that we would shoot one nominal weapon, say $20 \mathrm{kt}$, that would distribute the fission products uniformly over the Northern Hemisphere. Then we ignored inhalation and assumed that the iodine would enter the body via milk; and estimating the amount that a cow would ingest through grazing, we arrived at a figure for the lodine which we would expect to find in the urine samples. The estimates, on the order of $10 \mathrm{~d} / \mathrm{m} / 24-\mathrm{hr}$ sample, or less, indicated that we would have to use low-level counting techniques in order to determine the iodine in individual $24-\mathrm{hr}$ samples. We built a low-level counting apparatus which is arranged to accomodate four sample counters. It is surrounded by a large iron shield like the ones used for radiocarbon dating. Inside there is a bundle of Geiger tubes which completely surrounds the sample counter, or counters in some cases. The tubes are connected in anticoincidence to further reduce the background. We use a counter which is about 6 in. long and $1 \frac{1}{2} \mathrm{ln}$. in diameter, with its cylindrical wall being its sensitive surface. 
The apparatus gave us a background of about $3 \mathrm{c} / \mathrm{m}$. We were able to order the material for this apparatus, set it up, and have it operating in a month. Figure 1 shows the low-level counter.

In addition we have a 20-channel analyzer for use with a well-type scintillation counter so that we can do gamma analysis. Some of our screening work is done with a scintillation counter, and we count $\mathrm{Cs}^{137}$ with a scintillation spectrometer. However, our most precise work is done with our low-level beta counter.

The dots on Fig. 2 show the location of our collection stations throughout the United States. As I said, we did have a number of stations all over the world. Most of the stations were scattered about the Northern Hemisphere with a few in the Southern Hemisphere. At each station 10 individuals contributed 24-hr specimens once a week, from Tuesday to Wednesday morning. This gave us a total of about 2000 samples from the United States and about 500 samples from overseas, which we processed during the course of the Teapot serfes. We were unable to collect regularly from the overseas statlons since transportation was difficult and expensive. This work is supported by the Armed Forces Special Weapons Project.

\section{Urine Analyses for Fission Products}

The analytical procedure which we use for lodine is included at the end of the paper. It was written by one of the men in our group who was largely responsible for the chemical analyses. The procedure is a modiflcation of a procedure originally credited to Purves.* The iodine in the urine is exchanged on silver chloride, which is counted directly. The silver chloride is impregnated in asbestos mats which are wrapped around our counters, giving close cylindrical geometry. The lodine exchanges on the top surface of the mat, which is carefully prepared to give very little absorption. We beta-count, not gamma-count, the lodine.

During the course of this work we decided to save all the specimens and process them again for strontium. We did this and have again saved the specimens so that we may look for $\mathrm{Cs}^{137}$ in this urine. We looked at the $\mathrm{Sr}^{90}$ via the $\mathrm{Y}^{90}$ daughter since my assistant and I had worked with this procedure during the AEC Sunshine Project at the Chicago laboratory. We used the methods that Libby had invented, "in a few microseconds," as he said. The strontium procedure is given in detail with the iodine procedure.

Figure 3 shows the flow sheet for the present scheme of analysis, which we are using on the Redwing samples. The urine samples are preserved

* Purves, H. D., "Recovery of Radioactive Iodine from Urine," Nature 169, 111-112 (1952). 


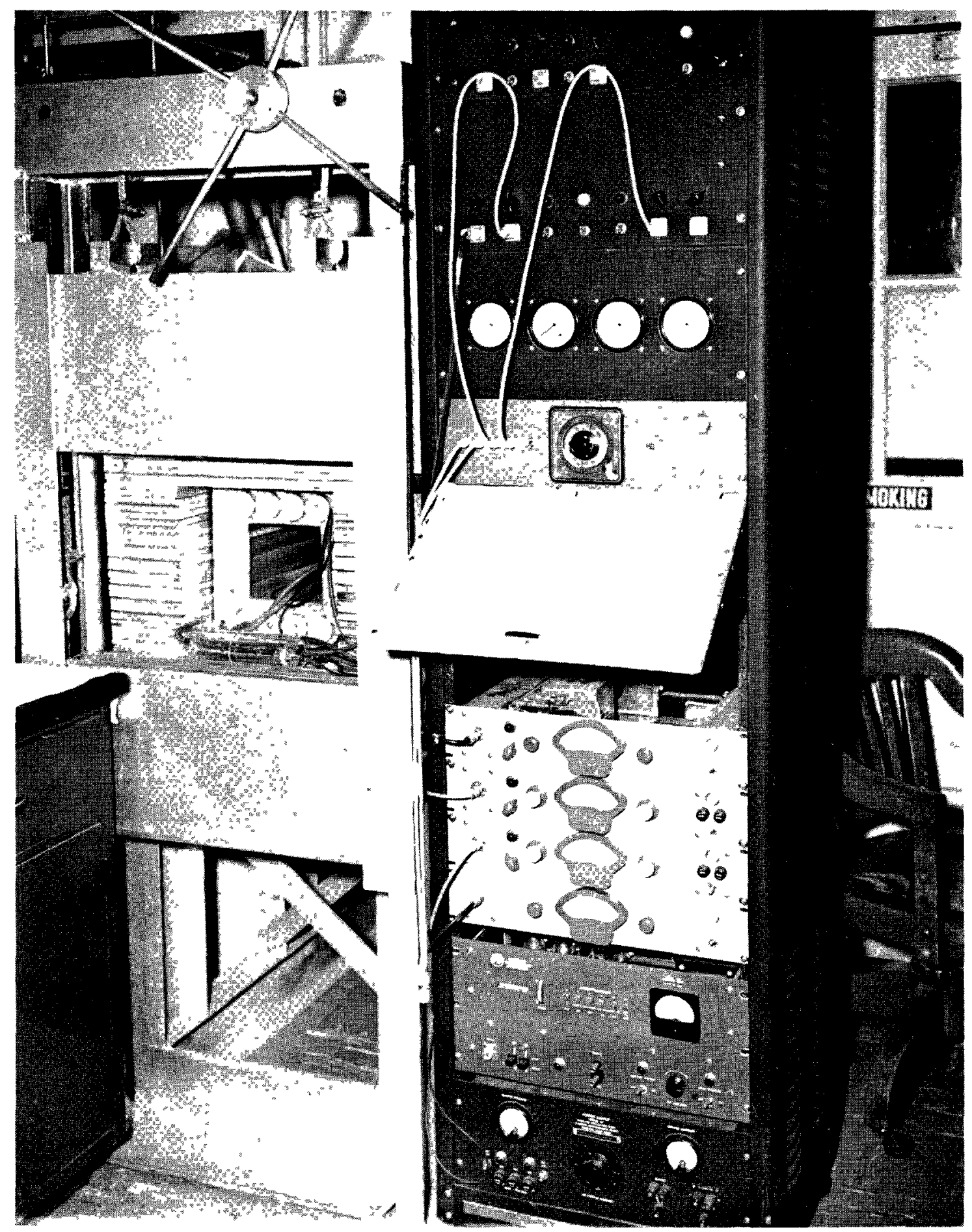

Fig. 1 Low-level beta counting apparatus. 


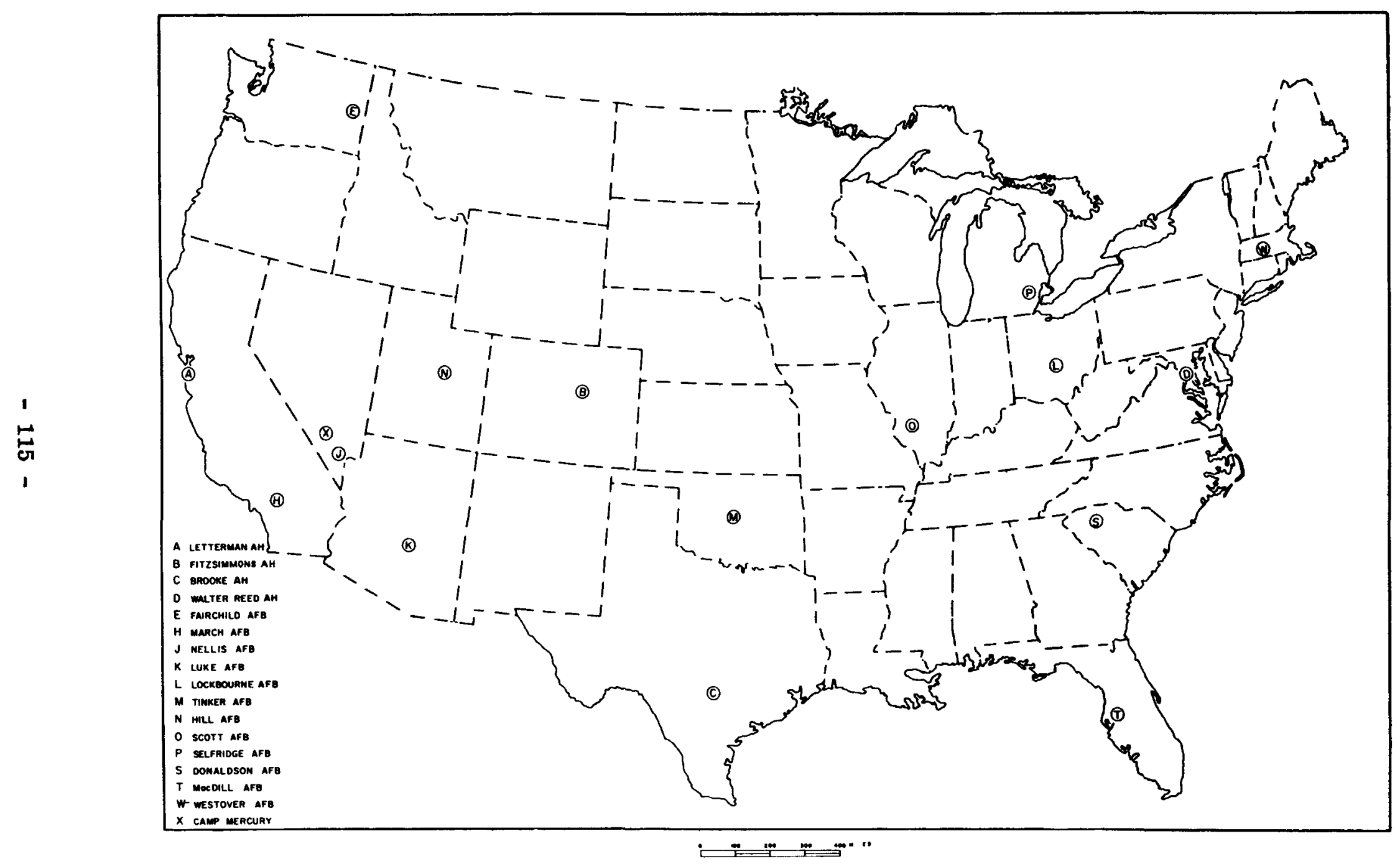

Fig. 2 Map of United States collection stations. 


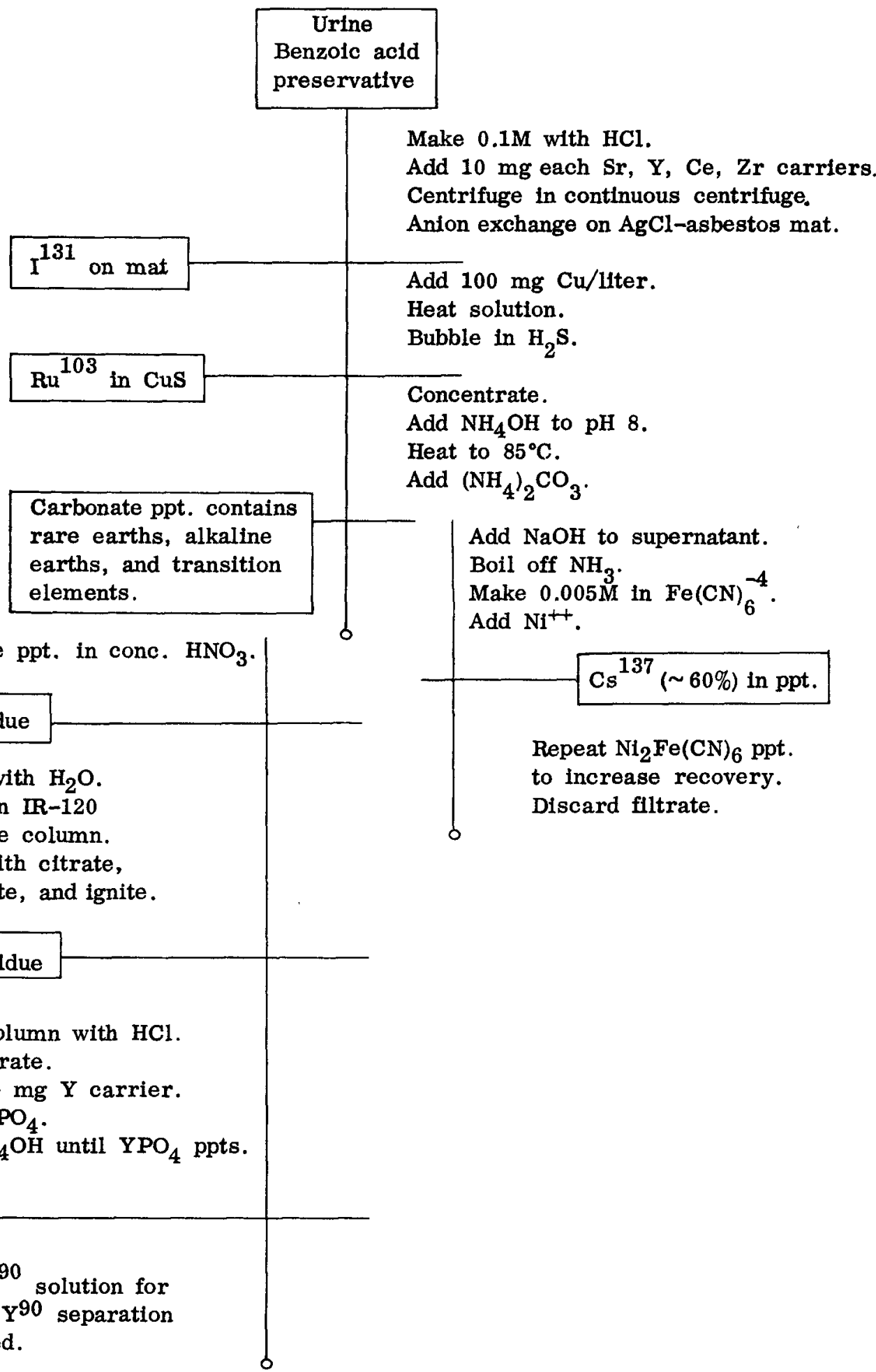

Elute column with $\mathrm{HCl}$.

Concentrate.

Add $200 \mathrm{mg} \mathrm{Y}$ carrier.

Add $\mathrm{H}_{3} \mathrm{PO}_{4}$.

Add $\mathrm{NH}_{4} \mathrm{OH}$ until $\mathrm{YPO}_{4}$ ppts.

$\mathrm{Y}^{90}$ in ppt.

Save $\mathrm{Sr}^{90}$ solution for further $\mathrm{Y}^{90}$ separation if needed.

Repeat $\mathrm{Ni}_{2} \mathrm{Fe}(\mathrm{CN})_{6} \mathrm{ppt}$ to increase recovery.

Dilute with $\mathrm{H}_{2} \mathrm{O}$.

exchange column.

Elute with citrate,

evaporate, and ignite.

$\mathrm{Ce}^{144}$ in residue

Fig. 3 Flow sheet for fission-product recovery from urine. 
with benzoic acid. The urine goes through anion exchange, giving us $\mathrm{I}^{131}$ on the mat, which we beta-count. This year we have counted the mats as quickly as possible. If we find appreciable activity, we continue with our complete procedure. If there is very little activity, we go directly to the alkaline earths and rare earths. If the complete procedure is indicated, we do a copper sulfide precipitation to bring down any ruthenium. Incidentally, we do not find much of this element. The levels this year are low, but we will be able to give some upper limits to the ruthenium that was excreted.

We have tried two procedures to reduce the urine to simple inorganic systems. One involved spray-drying followed by ignition of the residue. This gave us much trouble because we lost a lot of each sample in our spray dryer. The other was a concentration procedure, which actually worked very well, using a continuous-type, steam-heated, vacuum-concentration apparatus that recycled the urine. This enables us to concentrate the urine very rapidly.

The alkali metals are removed from the ignited samples by leaching. A carbonate precipitation is done on the concentrated urines, leaving the alkali metals in the filtrate. With either of these solutions we do a nickel ferrocyanide precipitation to bring down the cesium. If this precipitation is carefully done, we get about 80 per cent of the cesium. This precipitate is dissolved to separate the soluble cesium from the insoluble zirconium. The solution from the first precipitation contains the alkaline and the rare earths which were separated. The $\mathrm{Ce}^{144}$ in the rare-earth fraction is oxidized to the +4 state and precipitated as the iodate. Lanthanum-140 and $\mathrm{Y}^{90}$, the daughters of barium and strontium, are separated in the remaining rare-earth fraction and counted to calculate the amount of $\mathrm{Ba}^{140}$ and $\mathrm{Sr}^{90}$ present. This is, very roughly, the analytical scheme we are using this year.

We have decided that it is not worthwhile to examine for all these isotopes. We have found very little ruthenium or zirconium. We have found $\mathrm{I}^{131}, \mathrm{Ce}^{144}, \mathrm{Ba}^{140}, \mathrm{Sr}^{90}$, and their daughters. We do not usually measure the $\mathrm{Sr}^{89}$ which is present but assay $\mathrm{Sr}^{90}$ only. I can't give the detailed results of the analyses since we have not compiled the data for this year. I do have the results from the Teapot series for iodine and strontium. I will discuss these findings in some detail. As a matter of interest, the highest iodine excretion found in the Teapot series was $774 \pm 15 \mathrm{~d} / \mathrm{m}$, and the highest strontium level was $4.2 \times 10^{-6} \mu \mathrm{c}$, about $10 \mathrm{~d} / \mathrm{m} / 24-\mathrm{hr}$ sample. These samples were collected at Camp Mercury. Another sample from Mercury showed $160 \mathrm{~d} / \mathrm{m}$ of $\mathrm{I}^{131}$ and $3500 \mathrm{~d} / \mathrm{m}$ of $\mathrm{I}^{133}$ per $24-\mathrm{hr}$ sample. Incidentally, our over-all average volume on $24-\mathrm{hr}$ samples was $1250 \mathrm{ml}$. 
Figure 4 gives the data for samples collected at Camp Mercury during the Teapot series. Note that the dates or approximate times of the shots in the series and the corresponding sample-collection times are listed along the base. The data on these graphs are the average for several collected specimens. Individual samples varied considerably - by as much as a factor of 10, but the majority agreed quite well. The data on this, and subsequent figures, have been corrected to the time of collection. A week or more usually elapsed between the collection time and the time of analysis.

The results on samples collected at Salt Lake City are shown in Fig. 5. This graph includes the gummed-paper data which we obtained from the New York Operations Office. If the activity was less than 50 curles per square mile, we omitted it for that day. We have tried to correlate the activity in the urine with the cloud passage. The small " $x$ " denotes the passage of the cloud at the altitude shown over, or in the vicinity of, our collection station. The cloud data were supplied by the Weather Bureau. The correlation is not too good. At times the activity and cloud passage correlate well, and at times the activity increases when no radioactive cloud has passed near the station.

Figure 6 shows the data obtained at Oklahoma City. Again we have shown the cloud passage. We have not taken into account the spread of the cloud in these graphs. A cloud at this distance may be a few hundred miles wide, and although the center does not come near our station, our personnel may be exposed to some portion of it. In addition the winds are tricky and the material may be disseminated in various patterns. These figures show an increase in activity from the beginning to the end of the seriles. This year, during the Redwing series, we have not seen any striking increases in activity except in the cases of the pilots and the men who worked on the planes that actually flew through the cloud. Their samples did show a high level of activity. The highest average figure was something like $750 \mathrm{~d} / \mathrm{m} / 24-\mathrm{hr}$ sample.

Figure 7 shows data obtained at Walter Reed. You see that the gummed paper shows very little activity; of course, the activity in the Washington area was never high. Again, we find little correlation with cloud passage. More Washington data are shown in Fig. 8. This plot shows the urine data obtained at Walter Reed and the air-sampling data given to us by Blifford at the Naval Research Laboratory. Again, we did not get the correlation that we had hoped for between the biological data and the physical sampling.

The results obtained from the samples collected in Denver are shown in Fig. 9. It is of interest to note that during the 5 weeks between March 8 and April 12 the activity in the specimens dropped off with a 7-or 8-day 


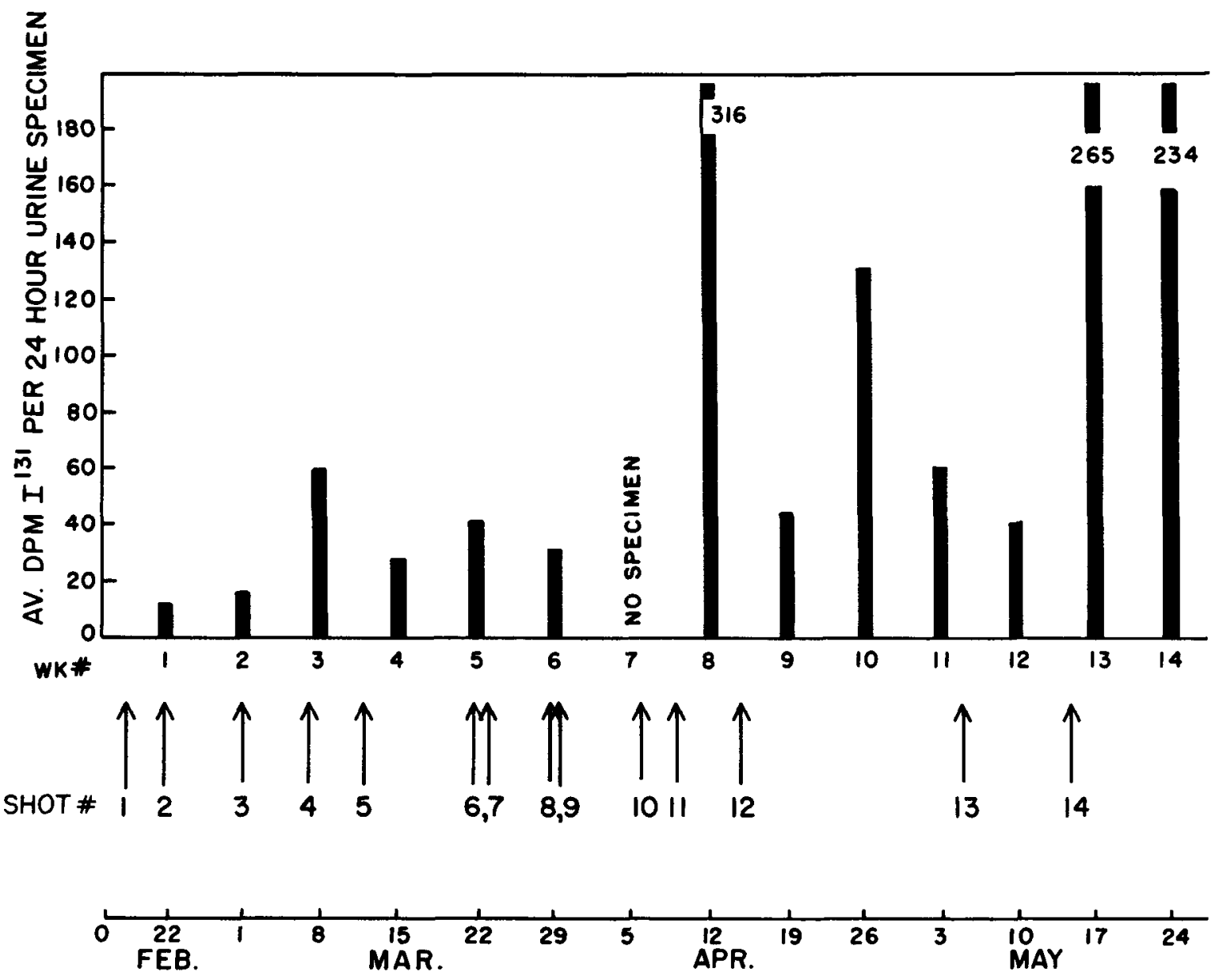

Fig. 4 Samples from Camp Mercury, Nevada. 

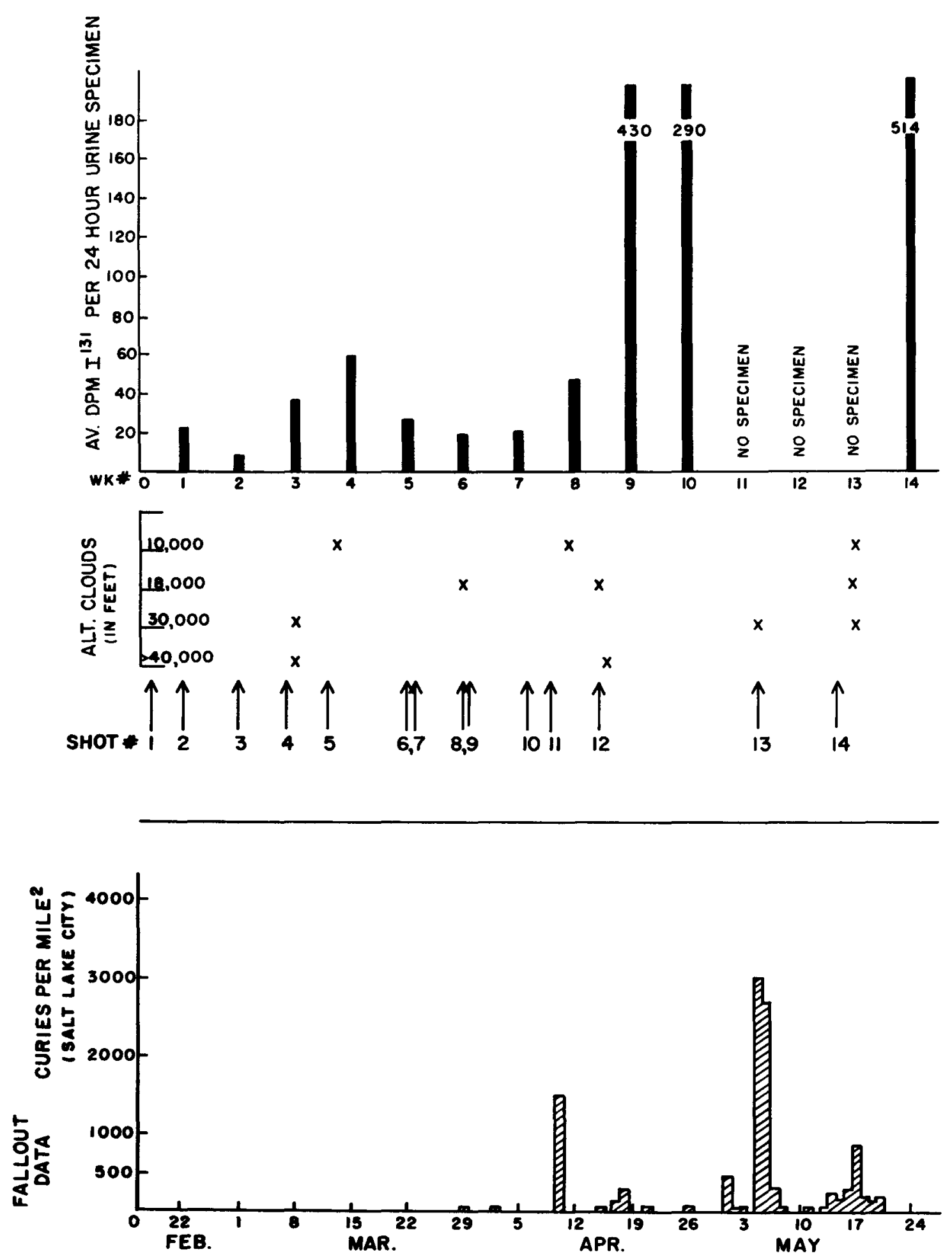

Fig. 5 Salt Lake City samples from Hill Air Force Base, Ogden, Utah. 

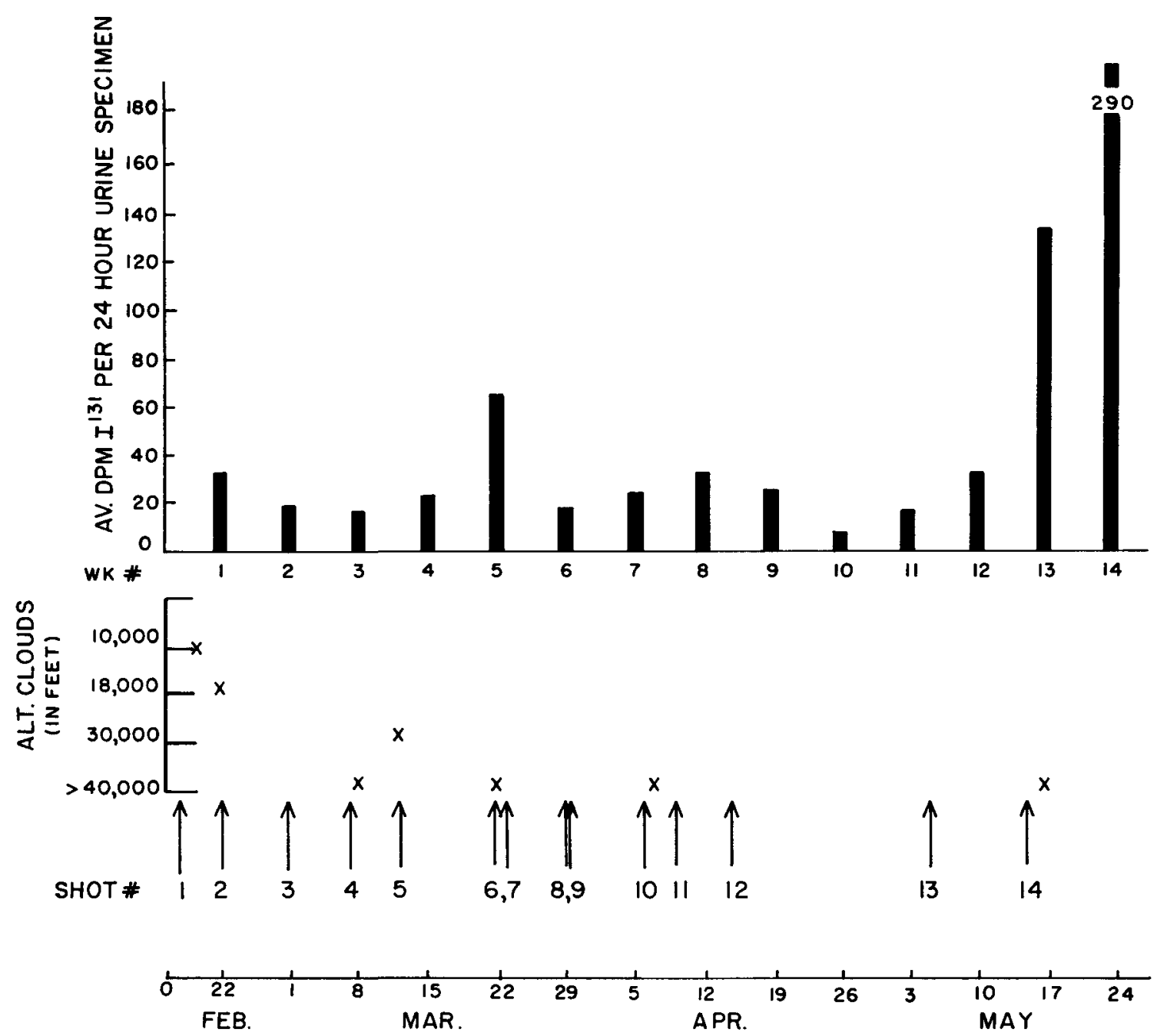

Fig. 6 Samples from Tinker Air Force Base, Oklahoma City, Oklahoma. 

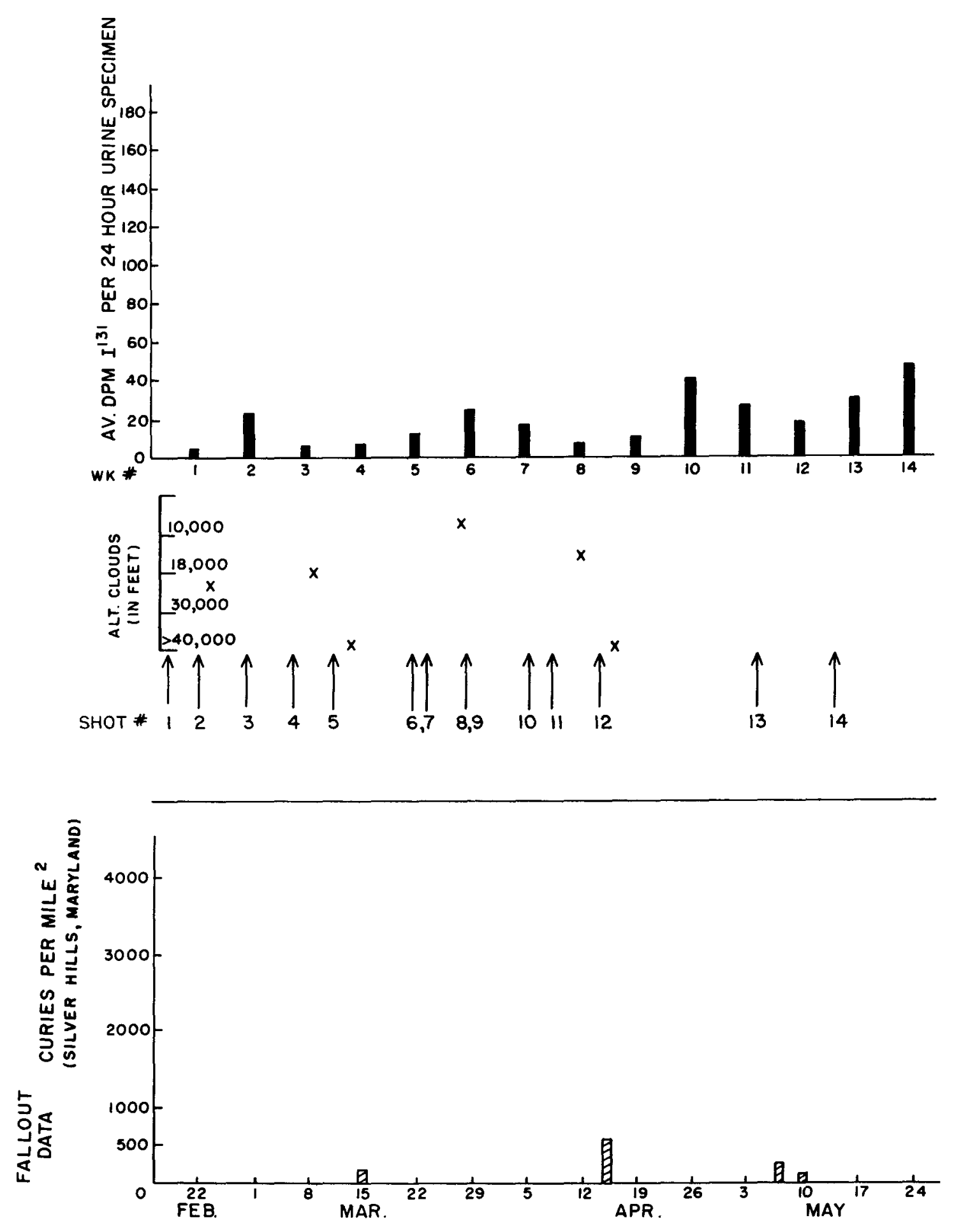

Fig. 7 Samples from Walter Reed Army Medical Center, Washington, D. C. 

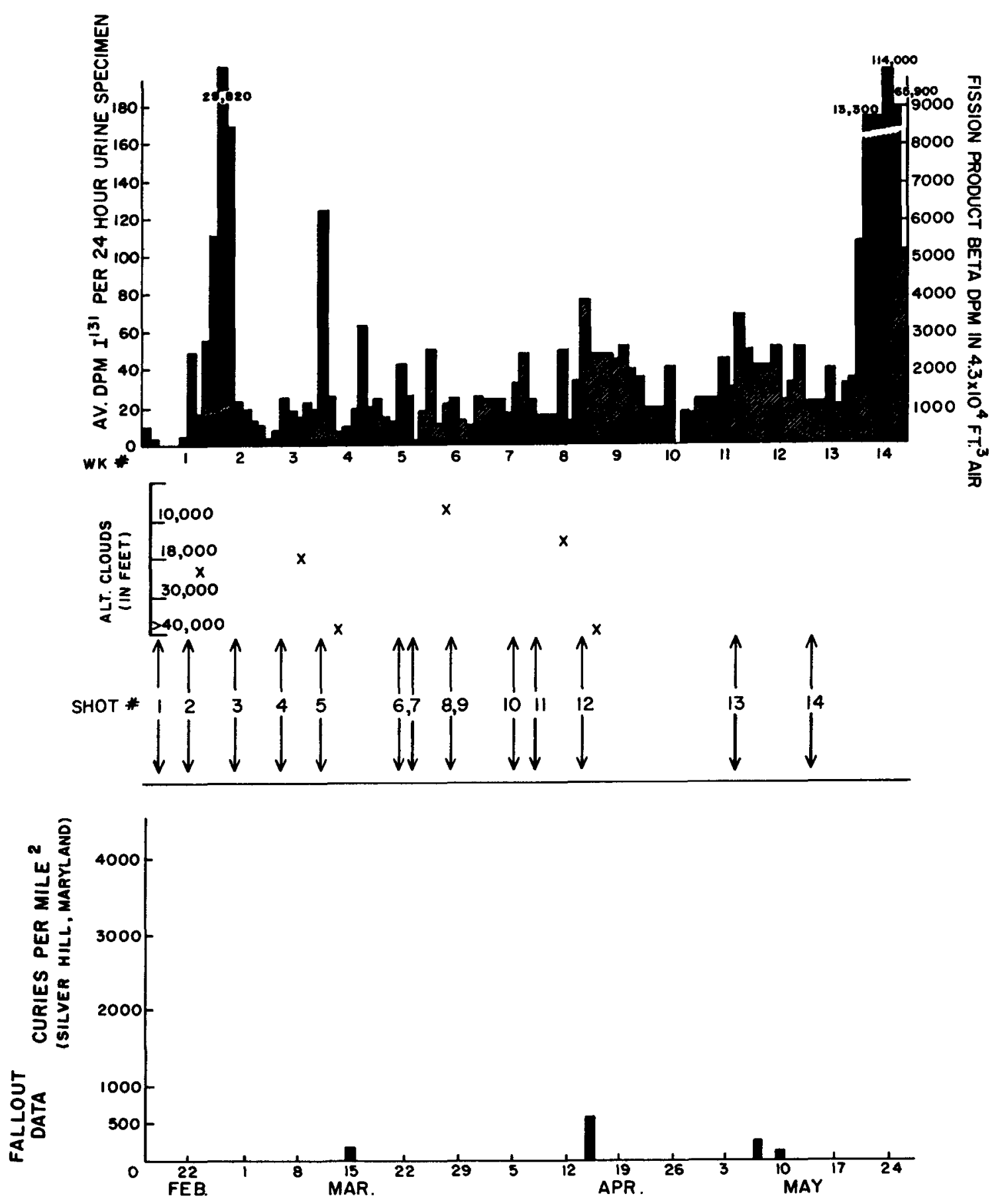

Fig. 8 Naval Research Laboratory air-sampling data (shaded area) superimposed on Fig. 7. 

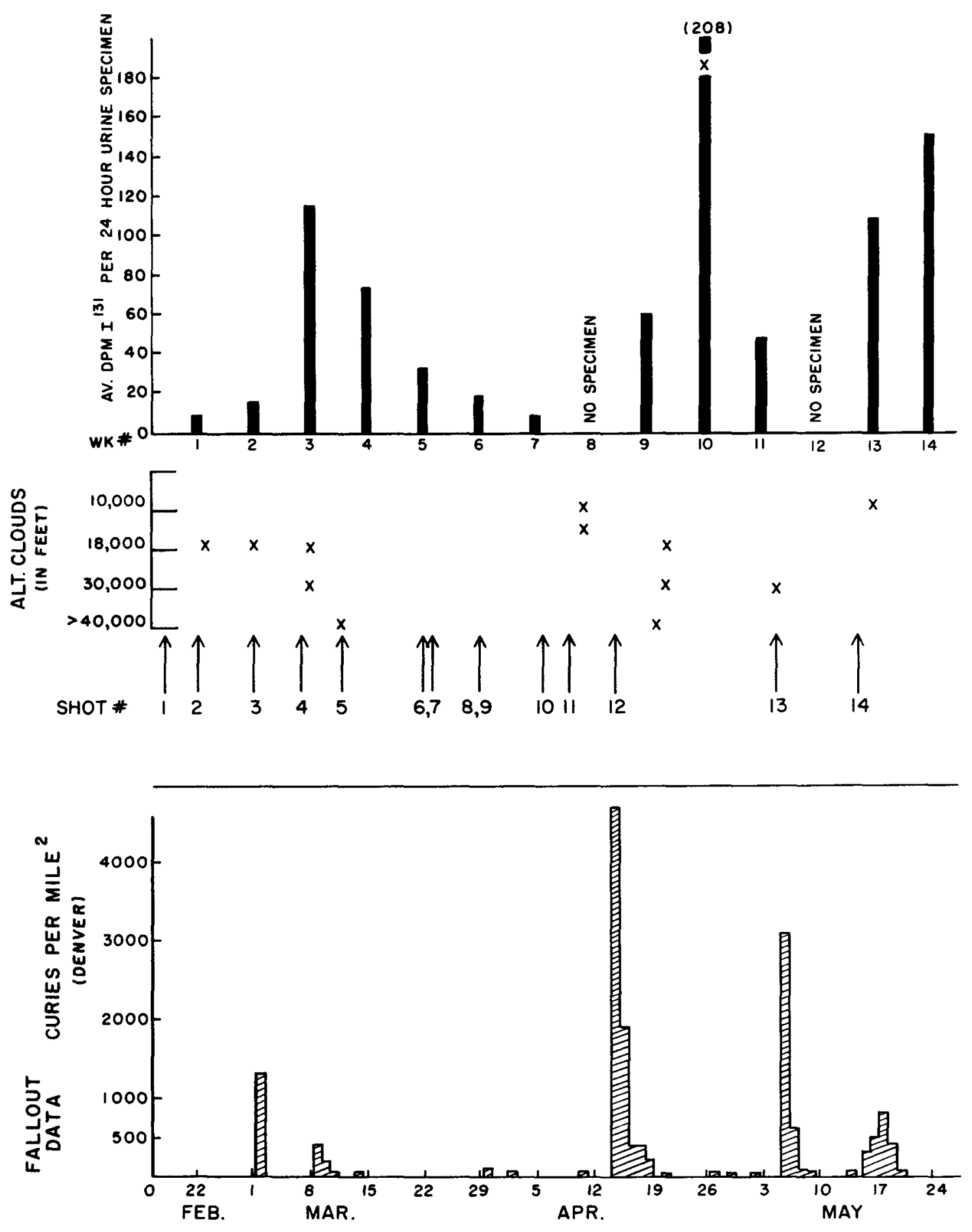

Fig. 9 Samples from Fitzsimmons Army Hospital, Denver, Colorado. 
half-life, which is close to that of the lodine itself. Again, this curve represents the average of the specimens obtalned from this station on these dates. It is al'so interesting to note that the average of the individual specimens plotted here gives a nice smooth curve, although the individuals vary a great deal. The activity excreted by an individual also varied from week to week, both increasing and decreasing. The curve shown here indicates that there must have been a continuing source of actlvity to which the people in this area were exposed, even though there were no clouds passing over this area for a period of 4 weeks.

$\underline{\text { Relationship Between } \mathrm{I}^{131} \text { and } \mathrm{Sr}^{90} \text { Activity }}$

The strontium data that we have is shown in Fig. 10. The strontium activity is much lower than that of lodine due to the long half-life of strontium. This plot also shows the $\mathrm{I}^{131}$ disintegrations which were corrected to the time of fission. The corrections may not be valid, but we did get a straight line which intersects the ordinate at 0 . Before correction the intersection was at 1250 on the ordinate. The strontium data represent combined samples. No one $24-\mathrm{hr}$ sample gave us enough strontlum activity to count, so we combined all the $24-\mathrm{hr}$ samples from a given station for one collection period. In some cases we had to combine all the samples for several weeks from one station in order to determine the strontium. From these we obtained an iodine:strontium ratio which is not unlike the ratio present in the fission-product mixture itself. We found a reasonable agreement, which may be simply fortuitous. In order to have background levels of the iodine and strontium activity in urine, we analyzed some combined samples before the test series began. We did find both lodine and strontium in these samples, with a higher ratio of strontium to iodine than found during the test. We are unable to explain the source of the iodine since there were no bombs fired, to our knowledge, at that time. Table 1 shows the $\mathrm{Sr}^{90}$ activity found in some pooled specimens.

Procedure for the Determination of $\mathrm{I}^{131}$ in Urine

The 24-hr urine samples, preserved with approximately $5 \mathrm{~g}$ of benzoic acid, were acidified with $3 \mathrm{ml}$ of concentrated sulfuric acid per liter of urine to bring the $\mathrm{pH}$ to approximately 1.0. The acidified samples settled overnight, and the sedimentary solids were removed by filtration through six to eight layers of filter paper.

An anion exchange mat was prepared using stock asbestos-silver chloride suspension. The stock suspension was made by combining in order: $1465 \mathrm{ml}$ of distilled water, $5 \mathrm{ml}$ of concentrated sulfuric acid, $25 \mathrm{ml}$ 


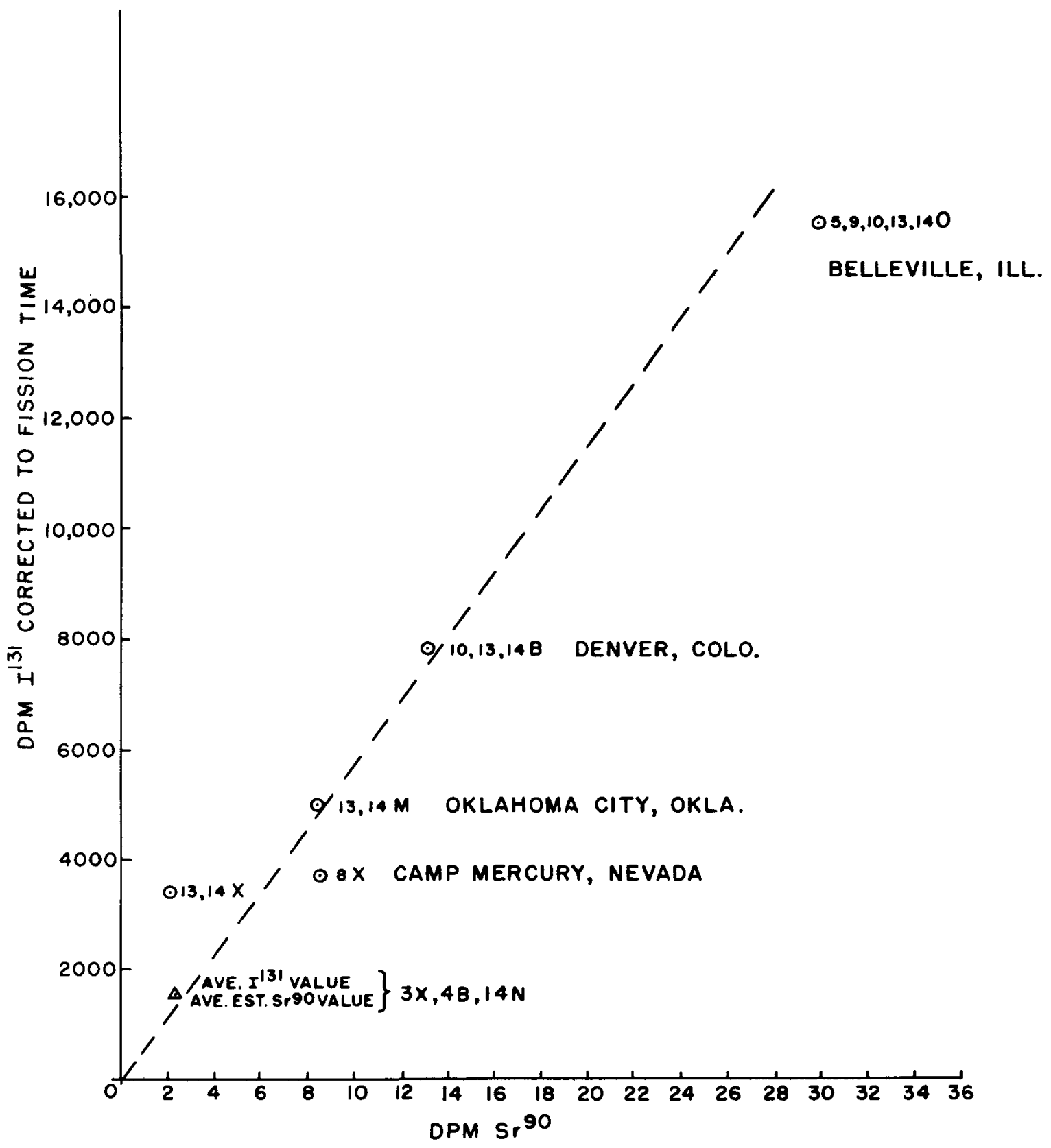

Fig. 10 Relationship between $\mathrm{I}^{131}$ and $\mathrm{Sr}^{90}$ activity (corrected data for pooled specimens by weeks and stations). 
Table 1

$\mathrm{Sr}^{90}$ IN TEAPOT URINE SAMPLES*

Sample

Overseas Combination \#1, pooled from Southern

Hemisphere stations,

collected 15 Feb. - 4 May 1955

Overseas Combination \#2, pooled

from northern Atlantic stations,

collected 15 Feb. - 4 May 1955

Overseas Combination \#3,

pooled from northern

Pacific stations,

collected 15 Feb. - 4 May 1955

Continental U. S. Combination \#1, pooled from U. S.

stations, collected 8 Feb. 1955

Denver, Colorado, pooled from 19 and

26 April 1955

Oklahoma City, Oklahoma, pooled from 19 and

26 April 1955

Camp Mercury, Nevada, from 15 Mar. 1955

* Additional data of this type will appear in a forthcoming report.
No. of 24-hr

Specimens

129

126

0.34

169

0.46

109

0.32

21

0.63

13

0.65

8

0.07

$$
\mathrm{d} / \mathrm{m} / 24-\mathrm{hr}
$$

(1)

8

of $1.25 \mathrm{~N}$ hydrochloric acid, and $30 \mathrm{~g}$ of Gooch asbestos. To this, $3.75 \mathrm{~g}$ of silver nitrate dissolved in $5 \mathrm{ml}$ of water was added dropwise with constant stirring.

Asbestos mats were prepared by assembling an $80-\mathrm{mm}$ medium-sintered glass filter funnel in a 2-liter filter flask. A filter paper, just large enough to cover the sinter, was put in place, and $100 \mathrm{ml}$ of the freshly prepared 
stock asbestos suspension was added. It was found that a battery of 12 mats could be made from each lot of the anion exchange preparation and operated on a manifold. It was necessary to shake the asbestos suspension frequently in order to mix it sufficiently to ensure a mat of uniform thickness. A filter paper was placed over the mat and suction was applied. When most of the liquid was drained from the mat, it was washed with two $25-\mathrm{ml}$ portions of $0.1 \mathrm{~N}$ sulfuric acid. Care was taken never to allow the mat to run dry since this was found to cause channeling within the mat.

When most of the second portion of wash liquid had been drawn through the mat, the urine was carefully poured to avoid disturbing the edges of the mat. Suction was regulated to a rate of flow not exceeding 1 liter per 10 min. When a few milliliters of urine remained, the mat was washed with two $25-\mathrm{ml}$ portions of $0.1 \mathrm{~N}$ sulfuric acid and sucked dry under full vacuum. The top filter paper was removed and discarded. The mat was then stripped from the filter funnel with a spatula and placed in a Petri dish.

Frequent use caused the funnels to clog and made control of filtration rate difficult. Haemosol was satisfactory for cleaning in moderate cases, and $6 \mathrm{~N}$ sulfuric acid was used for the more difficult.

Recovery studies of a series of mats indicated that the anion exchange process removed $90 \pm 10$ per cent of known amounts of iodine from the urine. This is of the same magnitude of iodine recovery as reported by Purves. Studies to determine the extent of self-absorption for beta radiation by the exchange mat were carried out. These demonstrated that approximately 80 per cent of the iodine was exchanged in the upper 10 per cent of the mat with a density of $4 \mathrm{mg}$ per square centimeter.

\section{Procedure for the Determination of $\mathrm{Sr}^{90}$ in Urine}

The 24-hr urine samples for each week from a station were pooled and acidified to $\mathrm{pH} 0.5$ with hydrochloric acid. Two grams of strontium nitrate and $30 \mathrm{~g}$ of calcium nitrate were added as carrier, together with $25 \mathrm{~g}$ of oxalic acid. The sample was heated to 80 or $90^{\circ} \mathrm{C}$. Oxalate salts were precipitated by adding sufficient concentrated ammonium hydroxide to approximate $\mathrm{pH} 8$, and the sample was allowed to stand overnight.

The following day most of the supernatant fluid was carefully siphoned off, and the remainder filtered by suction through a porcelain Buchner funnel containing Whatman No. 42 filter paper. The precipitate was pulled dry and washed with dilute ammonium oxalate-oxalic acid solution ( $2 \mathrm{~g}$ of ammonium oxalate per $100 \mathrm{ml}$ of 1 per cent oxalic acid). The precipitate was dried on the funnel, and paper was ignited overnight at $900^{\circ} \mathrm{C}$ in a porcelain crucible by an electric muffle furnace. 
The mixed oxides were welghed and dissolved in concentrated hydrochloric acid using approximately $3 \mathrm{ml}$ of acid per gram of oxide. The acid solution was diluted to approximately $3 \mathrm{~N}$ then filtered if not clear.

One milliliter of 85 per cent phosphoric acid per gram of oxide and $0.3070 \mathrm{gm}$ of yttrium oxide dissolved in dilute hydrochloric acid were added to the solution. The solution was then heated to 80 or $90^{\circ} \mathrm{C}$, and concentrated ammonium hydroxide was added slowly with vigorous stirring to redissolve any calcium phosphate which might be coprecipitated with yttrium phosphate. The base was added until slight opalescence was produced, and the solution was digested until the precipitate (yttrium phosphate) coagulated. Ten milliliters of $2 \mathrm{~N}$ ammonium hydroxide was added per milliliter of sample solution, and the solution was digested for 15 minutes. The precipitate was then recovered using Whatman No. 42 filter paper in a porcelain Buchner funnel.

The precipitate was dissolved from the funnel by two $10-\mathrm{ml}$ portions of hot $2 \mathrm{~N}$ hydrochloric acid followed with a wash of distilled water.

The combined solution and wash were diluted to approximately $400 \mathrm{ml}$. Five milliliters of 85 per cent phosphoric acid was added, and the solution was heated to 80 or $90^{\circ} \mathrm{C}$. Concentrated ammonium hydroxide was added until the solution was faintly opalescent. The precipitate (yttrium phosphate) was coagulated by digestion, and 50 to $75 \mathrm{ml}$ of $2 \mathrm{~N}$ ammonium hydroxide was added in small portions until the $\mathrm{pH}$ just changed from 1 to 2 as indicated by Hydrion paper. After a 15-min digestion, the solution was filtered as previously described.

The precipltate was pulled dry, transferred quantitatively to a porcelain crucible, and ignited by a gas-oxygen flame, while gently bleeding oxygen into the top of the crucible. The ignited sample was then weighed, ground to a fine powder, and mounted on a half cylinder of Bakelite for counting, using agar solution ( 0.1 per cent) and 95 per cent ethanol as mounting agents.

The filtrate from the final precipitation was alkalinized to approxtmately $\mathrm{pH} 8$ by the addition of ammonium hydroxide. The precipitate was separated by centrifugation, the supernatant decanted, and the precipitate dissolved in a few milliliters of hot $\left(70^{\circ} \mathrm{C}\right) 6 \mathrm{~N}$ hydrochloric acid. This solution was combined with the filtrate from the first yttrium phosphate precipitation. This solution, containing $\mathrm{Sr}^{90}$, was saved for subsequent "milkings" for the daughter yttrium.

It was noted from the decay curves of the single precipitation that 1sotopes other than $\mathrm{Y}^{90}$ were present. After a period of 2 weeks to allow equilibrium between $\mathrm{Sr}^{90}$ and $\mathrm{Y}^{90}$ to be approached, the solution was again processed to remove the yttrium. The only variation in the process was that the preliminary procedures through the first addition of phosphoric acid were 
not necessary. After the addition of the yttrlum oxide carrier, the process was as outlined above. In the event a fourth milking was deemed necessary, an excess of phosphate was assured by adding phosphortc actd. 


\title{
PROCEDURE FOR THE RADIOCHEMICAL ANALYSIS OF STRONTIUM AND BARIUM IN HUMAN URINE
}

\author{
L. B. Farabee
}

Oak Ridge National Laboratory

Union Carbide Nuclear Company

\section{Introduction}

I shall describe a procedure for the determination of radioactive strontium and barium in large volumes of urine. Strontium-90 is without doubt the most hazardous of the fission products due to its long half-life, its concentration in the bone, and the slow rate of elimination from the body. The urine of personnel, who are exposed to possible hazards of ingestion of fission products, should be analyzed periodically for $\mathrm{Sr}^{90}$. This procedure was designed to detect strontium in urine at low levels, and has been used routinely at Oak Ridge National Laboratory for two years.

A method for the routine analysis of radiostrontium in urine should be rapid and simple, and should provide for the removal of $\mathrm{K}^{40}$ and the organic and inorganic solids. This purification is necessary to eliminate self-absorption by the sample and the radiations from $\mathrm{K}^{40}$ in particular. Although this procedure is more time-consuming than one might wish, the method is (1) simple in operation, (2) specific for radiostrontium and radiobarium, and (3) excellent for separation from $\mathrm{K}^{40}$. Most of the operations are carrfed out on a cation exchange resin column. There is no necessity for adding either strontium or barium "carriers," which makes this procedure useful for analyzing either urine or bone for the presence of minute amounts of these elements in samples containing large amounts of calcium and magnesium.

The method depends on the coprecipitation of strontium and barium isotopes with the alkaline earth phosphate from an alkaline solution, the preferential chelation by Versene of calcium and magnesium over strontium and barium at a controlled $\mathrm{pH}$, the adsorption of ionic strontium and barium on a Dowex -50 resin column, the separation of adsorbed calcium and magnesium 
from the resin with a solution of citric acid and Versene of $\mathrm{pH} 5.0$, removal of the sodium from the resin with $0.5 \mathrm{~N}$ hydrochloric acid, and a final elution of the strontium and barium with $6 \mathrm{~N}$ nitric acid.

The Alkaline Earth Phosphate Precipitation

A short approach to a urinalysis procedure would be to use the urine directly without preliminary concentration by precipitation. However, certain difficulties arise in using urine directly with ion exchange resins in a separation procedure. The high concentration of dissolved salts, as well as the variability in individual specimens, and the presence of organic matter in urine makes a preliminary precipitation an important adjunct in this procedure. Strontium is coprecipitated with the calcium and magnesium in urine as an alkaline earth phosphate in a basic medium. This operation is simple and effectively separates the alkaline earths from the sodium, potassium, and organic matter, and eliminates the laborious process of ashing large volumes of urine. After centrifugation of the phosphate precipitate and subsequent destruction of the remaining organic material, the chief difficulty is the separation of radiostrontium from the calcium and magnesium normally present in a urine sample.

Because of this large amount of calcium and magnesium, conventional methods of separating the radiostrontium are difficult to apply in urinalysis. The addition of strontium "carrier" and the precipitation of strontium nitrate from a 75 to 80 per cent nitric acid solution has the disadvantages that (1) the acid is very corrosive, (2) several precipitations are necessary with some loss of strontium in each, and (3) gravimetric determination of the recovered strontium "carrier" is complicated by the presence of an indeterminate amount of nonradioactive strontium that is excreted in the urine. The strontium chromate precipitation from a buffered acetate solution at a $\mathrm{pH}$ of 8 in the presence of 35 per cent alcohol was the basis of a procedure which was developed at ORNL in 1949. This was published as "A Procedure for the Radiochemical Analysis of Barium, Strontium, and the Rare Earths in Urine" in ORNL-368 and in AECU-2692. Cation exchange resins were used to separate the phosphates and sulfates prior to the chromate precipitation in a basic medium. This procedure is long and complicated and the chromate precipitate is difficult to handle. Some of the above difficulties have been circumvented by this new strontium procedure which uses Dowex-50 resin and Versene and sodium citrate as complexing agents. 
Preparation of the Column

Beds of $50-100$ mesh Dowex-50-X12* are prepared in a Pyrex glass column $18.5 \mathrm{~cm}$ long and $1.8 \mathrm{~cm} \mathrm{I.D.} \mathrm{The} \mathrm{bottom} \mathrm{is} \mathrm{fitted} \mathrm{with} \mathrm{a} \mathrm{one-way}$ stopcock in a one-hole rubber stopper. Glass wool over the rubber stopper holds the resin bed. A 6-in. Pyrex funnel fitted to the top of the glass column with a rubber tubing serves as a reservoir for the feed and wash solutions. The Dowex -50 resin is converted to the sodium cycle by regenerating with sodium chloride. The resin which has been used in urinalysis can be re-used by converting to the sodium cycle.

It was expedient to keep the amount of resin used to a minimum in order to reduce the volume of elutriant solutions and thereby reduce the time required to perform a single analysis. Experimentally, it was found that $16.3 \mathrm{~g}$ of resin (air-dried) or about $25 \mathrm{ml}$ by volume (wet-form) was sufficient for an individual analysis.

\section{Operation of the Column}

The planning and interpretations of ion exchange separation schemes are dependent upon a knowledge of the interactions among the adsorbing ions, the eluting ions and the exchanger. Most ion exchange separations are performed under nonequilibrium conditions. However, a knowledge of equilibrium states furnishes a rapid and reliable method for evaluating the effect that chemical variables will have upon nonequilibrium operations.

Equilibrium studies show that (1) the affinity of a cation exchanger for the alkaline earths will be: ${ }^{1} \mathrm{Ba}^{++}>\mathrm{Sr}^{++}>\mathrm{Ca}^{++}>\mathrm{Mg}^{++}$, (2) the ease of removal of the alkaline earths by citrate solutions is just the reverse, and (3) alkaline earths vary in the ease by which they are chelated by Versene as indicated by their stability constants. ${ }^{2}$ From such data one would expect the order of elution from a cation exchange resin to be $\mathrm{Ca}^{++}>\mathrm{Mg}^{++}>\mathrm{Sr}^{++}>$ $\mathrm{Ba}^{++}$. The difference in the adsorption of calcium and strontium on Dowex-50 resin in the presence of excess Versene is shown in Fig. 1. It will be noted that Versene becomes a less efficient chelating agent as the $\mathrm{pH}$ is decreased, and is also more effective for chelating calcium than strontium in the $\mathrm{pH}$ range 4.5 to 6.0 .

This urinalysis procedure was designed to handle up to $800 \mathrm{mg}$ of calcium and $200 \mathrm{mg}$ of magnesium per sample. This is more calcium than is excreted per day. The strontium analysis is made on the supernate after plutonium separation, where $600 \mathrm{mg}$ of calcium is added to the urine sample

*Product of Dow Chemical Co., Midland, Mich. 


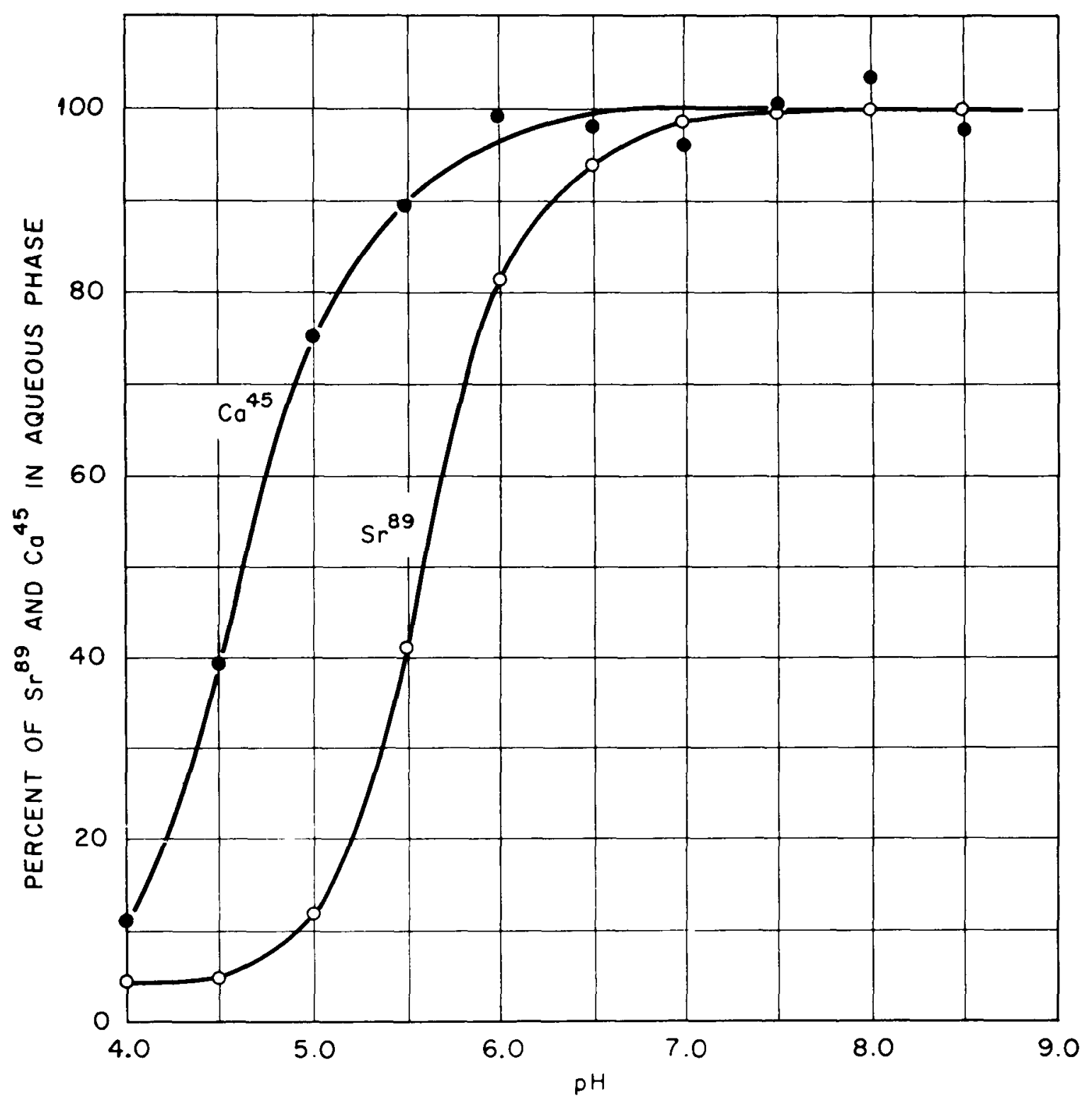

Fig. 1 Distribution of carrier-free $\mathrm{Sr}^{89}$ and $\mathrm{Ca}^{45}$ in a 1 per cent solution of disodium dihydrogen Versenate equilibrated with $1 \mathrm{~g}$ of Dowex-50 (50 to $100 \mathrm{mesh}$ ) as a function of $\mathrm{pH}$. 
prior to the plutonium analysis. It was expedient to use one urine sample for both plutonium and strontium analyses. This quantity of alkaline earths would exceed the capacity of the resin column described above. Therefore, it was necessary to minimize the adsorption by adding sufficient Versene to chelate all of the alkaline earths, and adjusting the $\mathrm{pH}$ of the solution to 5.5 prior to putting this feed solution over the resin column. In this manner, 97 per cent of the calcium and about 50 per cent of the magnesium will pass through the resin bound in nonionic form as a Versenate chelate, while the ionic strontium and barium are retained on the resin. The effluent loss of $\mathrm{Sr}^{89}$ was less than 0.2 per cent.

As shown in Fig. 1 Versene is a less efficient chelating agent as the $\mathrm{pH}$ is reduced; therefore, there will be some calcium and magnesium adsorbed on the resin when the pH of the feed solution is reduced to 5.5. From experimental runs it was found that 15 to $25 \mathrm{mg}$ of calcium and 40 to $50 \mathrm{mg}$ of magnesium remained on the resin column. This residual calcium and magnesium can be moved from the resin, without loss of strontium, by passing over the column an elutriant solution composed of a mixture of 0.75 per cent technical grade Versene - 1 per cent citric acid adjusted to a $\mathrm{pH}$ of 5.0 with sodium hydroxide. The calcium is removed by the Versene while the magnesium is removed by the citrate. The effectiveness of various eluting solutions for the removal of calcium and magnesium from a Dowex-50 resin column is shown in Figs. 2, 3, and 4.

After the removal of the calcium and magnesium, only strontium, barium and sodium remain on the column. Since the Dowex -50 resin had been put on the sodium cycle in preparation for urinalysis, the sodium must be removed in order that the final eluate can be evaporated and prepared for counting without further chemical purification. Eight hundred milliliter of $0.5 \mathrm{~N}$ hydrochloric acid put over the column at a flow rate of $4 \pm 0.8$ $\mathrm{ml} / \mathrm{min} / \mathrm{cm}^{2}$ will elute all of the sodium and only about 0.2 per cent of the strontium.

Elution and Preparation for Counting

The final step is the elution of the strontium and barium from the resin column. This is done with $200 \mathrm{ml}$ of $6 \mathrm{~N}$ nitric acid at a flow rate of $2 \mathrm{ml} / \mathrm{min} / \mathrm{cm}^{2}$. This volume of acid will elute 99.6 per cent of the strontium and barium. The eluate is evaporated to near dryness at a temperature slightly below the boiling point and is transferred to a stainless steel dish for counting. The density of the inorganic residue in the counting dish will be less than $1 \mathrm{mg} / \mathrm{cm}^{2}$; therefore, self-absorption of the beta radiation by the sample will be nil. 

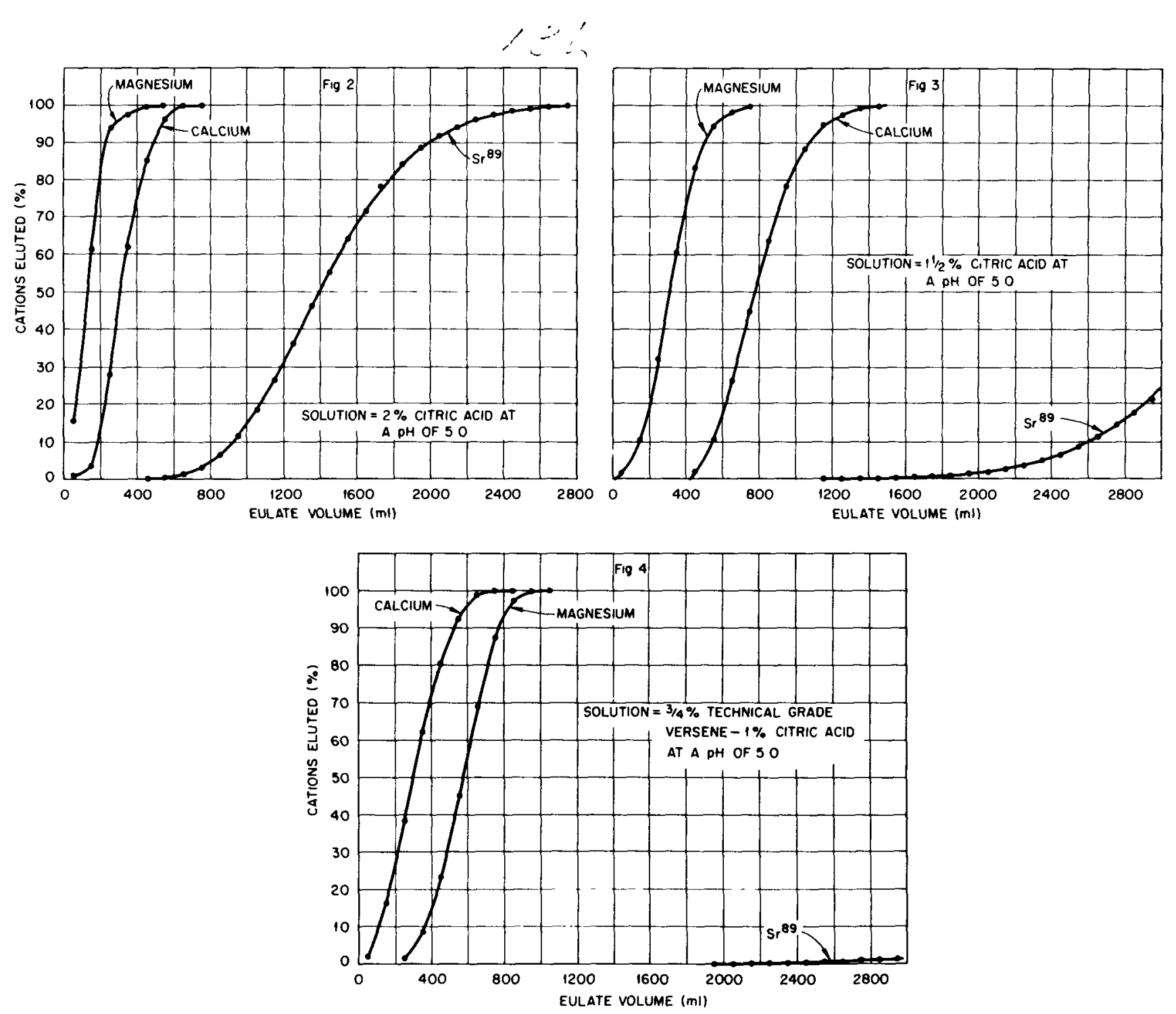

Figs. 2, 3, 4 Elution of a mixture of $50 \mathrm{mg}$ of magnesium, $25 \mathrm{mg}$ of calcium, and about $10^{6} \mathrm{c} / \mathrm{m}$ of $\mathrm{Sr}^{89}$ from a column $18.5 \mathrm{~cm}$ long and $1.8 \mathrm{~cm} \mathrm{I}$. D. containing $16.3 \mathrm{~g}$ of Dowex-50-X12 (air-dried and on sodium cycle). Flow rate, $4 \mathrm{ml} / \mathrm{min} / \mathrm{cm}^{2}$. 
Separation from Other Fission Products and Also $\mathrm{K}^{40}$

The determination of radioactive strontium in urine at low levels requires complete separation from fission products and also $K^{40}$. This procedure provides a separation factor of $3 \times 10^{4}$ from fission products other than strontlum and barium. A 24-hr urine specimen will contain about $3400 \mathrm{~d} / \mathrm{m}$ beta due to $\mathrm{K}^{40}$. Using this procedure on urine samples from 17 persons who had no known exposure to fission products, the average beta activity was $2.8 \mathrm{~d} / \mathrm{m}$.

\section{$\underline{\text { Results }}$}

More detailed studies of the various steps indicated that the average loss of $\mathrm{Sr}^{89}$ tracer in the phosphate supernate is less than 0.5 per cent, while the total losses in the column operation are less than 1 per cent. At $\mathrm{Sr}^{89}$ tracer levels of about $75 \mathrm{c} / \mathrm{m}$, the recovery in 22 runs was reproducible to \pm 2.5 per cent. Although it would be desirable that this procedure be specific for strontium, radioactive barium is recovered along with strontium. However, due to its shorter half-life the probability that radiobarium will be present in a urine sample is small. This procedure is simple to carry out in ordinary laboratory operation, and there are no critical steps where strontium loss is liable to occur. The time for an individual analysis is long, but one operator can do from 8 to 10 analyses at one time. Since the chemical recovery of strontium is about 98 per cent, no gravimetric determination is necessary. This procedure may find application to the radiochemical analysis for strontium in other media in which large amounts of calcium are present.

\section{References}

1. Nachod, F. C., Ion Exchange - Theory and Application, p. 175, Academic Press, New York, 1949.

2. Martell, A. E., and Calvin, Melvin, Chemistry of the Metal Chelate Compounds, p. 445, Prentice-Hall, Inc., New York, 1952.

Questions and Answers

Question: How often do you sample your personnel on a routine basis and what sort of urine tolerance levels do you use? 
Answer: I would like to ask P. E. Brown, who is in charge of the routine urinalysis program at ORNL, to answer these questions.

Mr. Brown: On a routine basis we try to obtain one sample per year from each exposed man. When a man is working with high levels of activity we collect weekly samples. In cases of unusually high exposures we collect more often. Our criteria for $\mathbf{S r}^{89}$ is $400 \mathrm{~d} / \mathrm{m} / 24-\mathrm{hr}$ sample. This is based on a $2-\mu \mathrm{c}$ body burden and an effective half-life of 2700 days as given in the ICRP Report of 1954 or NBS Handbook 52.

Question: Have you any data on the strontium excretion pattern following high exposures?

Answer: In our experience with high human exposures, we have found that the excretion is very rapid at first and then drops off and seems to approach an exponential rate. After about 180 days you have something on the order of a 60-day excretion half-life. After 300 or 1000 days the half-life levels off to something like 1000 days. The various half-lives give you a series of exponentials. We tried fitting these data to power functions and found a reasonable fit after the first 4 days. The power function does not fit the excretion curve during the first 4 days. After a long period of time (after exposure) we have found anomalies in the curve which may be due to re-exposures or perhaps to a hold-up somewhere in the system. C. L. Comar, at ORINS, has data on sheep which show the same excretion pattern and power function. However, with his data we were able to show a continuous straight line to about 500 days, where his data ended. There were no anomalies in the curve.

Question: Do you have data on the comparison of fecal to urine excretion of strontium?

Answer: We have no human data on the feces: urine ratio, but the sheep data indicate about a $1: 1$ ratio. This does not hold true during the first 4 days. The sheep showed something like 20 per cent of the total dose excreted in the first day. Most of this was in the urine. On the second day a total of more than 20 per cent was excreted, and after that the percentage of the total went down and then began leveling off to a very long half-life.

Question: What type of power function did these curves follow?

Answer: The power function that Dr. Langham showed: 


$$
\frac{d R_{t}}{d t}=-A b t^{-(b+1)}
$$

Procedure for the Analysis of Urine for Strontium and Barium*

The Alkaline Phosphate Precipitation

1. Add concentrated hydrochloric acid to a $1500-\mathrm{ml}$ urine sample to make the urine $0.1 \mathrm{~N}$ in hydrochloric acid and yleld a clean solution.

2. Heat the sample on a hot plate to a temperature of 85 to $90^{\circ} \mathrm{C}$.

3. Add $6 \mathrm{ml}$ of $6 \mathrm{M}$ phosphoric acid to provide an excess of phosphate to insure complete precipitation of all calcium and magnesium.

4. Use an electric motor stirrer to provide vigorous stirring for the subsequent precipitation.

5. Add slowly $6 \mathrm{M}$ sodium hydroxide until a basic phosphate precipitate is visible. Continue the addition until the solution is basic, $\mathrm{pH}$ of 8 to 10 . Universal $\mathrm{pH}$ paper can be used to determine the $\mathrm{pH}$ in this case.

6. Allow the precipitate to settle for $2 \mathrm{hr}$ or longer.

7. Decant the supernatant liquid by suction to the lowest possible level without disturbing the precipitate. Discard the supernatant solution.

8. Pour the remaining slurry into a centrifuge cup. Centrifuge the precipitate at $1500 \mathrm{rpm}$ for $5 \mathrm{~min}$. Decant the supernatant liquid by suction and discard.

Preparation of the Column

(a) Description of column system

Beds of 50-100 mesh Dowex-50-X12 resin are prepared in a Pyrex glass column $18.5 \mathrm{~cm}$ long and $1.8 \mathrm{~cm} \mathrm{I.D.} \mathrm{The} \mathrm{bottom} \mathrm{of} \mathrm{the} \mathrm{glass} \mathrm{column}$ is fitted with a one-way stopcock in a one-hole rubber stopper. Glass wool over the rubber stopper holds the resin bed. A 6 in. Pyrex funnel fitted to

*The procedure given here is taken from the document "Procedure for the Radio-chemical Analysis of Strontium and Barium in Human Urine," Oak Ridge National Laboratory, Report ORNL-1932, September 1955. 
the top of the glass column with a rubber tubing serves as reservoir for the feed and wash solutions.

\section{(b) Preparation of the resin}

New Dowex -50 resin is conditioned by several washings, alternating 5 per cent sodium chloride and 5 per cent hydrochloric acid. During the conditioning process a considerable quantity of "fines" is removed. This is accomplished by stirring the resin sample in a large beaker filled with the conditioning solution. Most of the resin is allowed to settle and the liquid containing the "fines" is decanted. The resin is then converted to a sodium cycle with 5 per cent sodium chloride. About $250 \mathrm{~g}$ of the resin is put into a Pyrex glass tube $4 \mathrm{ft}$ long and $2.5 \mathrm{~cm}$ in diameter. Six liters of 5 per cent sodium chloride is then passed over the resin at a maximum flow rate $\left(4.7 \mathrm{ml} / \mathrm{min} / \mathrm{cm}^{2}\right)$. The resin is emptied into a large beaker and washed free of sodium chloride with distilled water. The resin which has been used in urinalysis can be re-used by converting it to the sodium cycle as described above.

Operation of the Column

(a) Preparation of feed solution

1. Dissolve the precipitate from the alkaline phosphate precipitation, Step 8, in about $15 \mathrm{ml}$ of concentrated nitric acid.

2. Pour this solution into the beaker in which the original phosphate precipitation was carried out.

3. Destroy the organic matter by heating the solution and by alternately adding concentrated nitric acid and 30 per cent hydrogen peroxide.

4. Repeat Step 3 until a white residue remains and take to dryness.

5. Remove the beaker from the hot plate and wash down the walls with about $20 \mathrm{ml}$ of distilled water. Add 2 to $3 \mathrm{ml}$ of concentrated hydrochloric acid and heat until the inorganic material is dissolved. Dilute to $800 \mathrm{ml}$ with distilled water.

6. Add $1 \mathrm{~N}$ sodium hydroxide until the $\mathrm{pH}$ is about 9.0 .

7. Add $2 \mathrm{ml}$ Eriochrome Black T.*

* Mix $0.5 \mathrm{~g}$ of Eriochrome Black $\mathrm{T}$ and $4.5 \mathrm{~g}$ of hydroxylamine hydrochloride. Dissolve in $100 \mathrm{ml}$ of alcohol and filter. 
8. Add a solution of 7.5 per cent* technical grade Versene until the indicator changes from wine red to blue at pH 10.5. This point indicates complete chelation of all calcium and magnesium.

9. Reduce the $\mathrm{pH}$ to 5.5 with concentrated hydrochloric acid. The final adjustment can be made with $1 \mathrm{~N}$ hydrochloric acid.

10. Pass this solution over the resin column at a flow rate of not greater than $8 \mathrm{ml} / \mathrm{min} / \mathrm{cm}^{2}$.

11. Wash down the walls of the funnel with about $50 \mathrm{ml}$ of distilled water.

12. Discard the effluents.

(b) Elution of adsorbed calcium and magnesium from the resin column

1. Pass $800 \mathrm{ml}$ of a solution of 1 per cent citric acid and 0.75 per cent Versene $(10.93 \mathrm{~g}$ of citric acid monohydrate, $100 \mathrm{ml}$ of 7.5 per cent Versene, diluted to 1 liter and the $\mathrm{pH}$ adjusted to 5.0 with $6 \mathrm{M}$ sodium hydroxide) over the resin column at a flow rate of $4 \pm 0.8 \mathrm{ml} / \mathrm{min} / \mathrm{cm}^{2}$.

2. Discard the effluent wash.

(c) Removal of sodium from the resin column

1. Pass $800 \mathrm{ml}$ of $0.5 \mathrm{~N}$ hydrochloric acid over the resin column at a flow rate of $4 \pm 0.8 \mathrm{ml} / \mathrm{min} / \mathrm{cm}^{2}$.

2. Discard the effluent wash.

(d) Elution of strontium and barium

1. Pass $200 \mathrm{ml}$ of $6 \mathrm{~N}$ nitric acid over the resin at a flow rate of $2 \mathrm{ml} / \mathrm{min} / \mathrm{cm}^{2}$.

2. Catch the eluate in a $400-\mathrm{ml}$ beaker.

Preparation of the Sample for Counting

1. Evaporate the nitric acid eluate to near dryness on a hot plate.

2. Wash the contents into a $50-\mathrm{ml}$ beaker with distilled water. Clean the walls of the larger beaker with nitric acid and water and add the wash

*Dissolve $75 \mathrm{~g}$ of tetrasodium ethylenediaminetetraacetic acid (technical grade) in about $800 \mathrm{ml}$ of distilled water. Filter and dilute to 1 liter. 
to the small beaker.

3. Evaporate the liquid in the small beaker to dryness.

4. Dissolve the residue in about $0.5 \mathrm{ml}$ of $1 \mathrm{~N}$ nitric acid. Transfer this liquid to a counting dish* using a transfer pipette. Wash the walls of the beaker with another $0.5 \mathrm{ml}$ of acid, and add this to the dish.

5. Dry under an infra-red lamp.

The radioactivity of the sample can be counted in a conventional endwindow Geiger-Mueller counter.

*This counting dish is made from a circular piece of type 304 stainless steel 1-3/4 in. in diameter and 0.005 in. thick. A cup, 1 in. in diameter and 0.125 in. in depth, is die-pressed for holding the sample. 


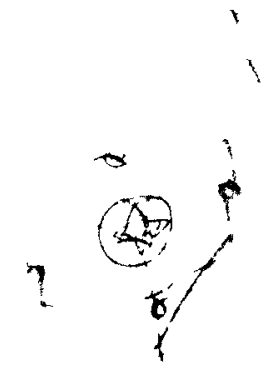

THE HUMAN COUNTER

E. C. Anderson

Los Alamos Scientific Laboratory

University of California

Los Alamos, New Mexico

The difficulties in determining body burdens based on chemical analyses of the excreta aroused our interest in developing an alternative technique, the whole body counter. The advantages are obvious. The question of excretion rate and the problem of extrapolation from excretion to body burden are eliminated, no chemical separations are necessary, and the procedure is extremely rapid. The limitations are also obvious. The only isotopes which can be detected are gamma emitters and, possibly, those that emit $x$-rays. The method may be applicable to bremsstrahlung detection of hardbeta emitters such as the $\mathrm{Sr}^{90}-\mathrm{Y}^{90}$ pair, but there is a severe loss of sensitivity with isotopes other than gamma emitters.

\section{The Counter}

Two systems have been developed for whole body counting. One is the system developed by L. D. Marinelli ${ }^{1,2}$ at the Argonne National Laboratory which uses a large sodium iodide crystal in a specially shielded low-background chamber. The NaI crystal has some pronounced advantages. The energy resolution is very high and it has a wide detection range. It can detect gamma energies as low as $40 \mathrm{Kev}$. Marinelli has shown that it is possible to determine a number of gamma-emitting isotopes simultaneously. In addition it is possible to localize activity within the body using the NaI crystal. On the other hand, there are disadvantages in the use of this method. Sodium iodide crystals are limited in size and it is difficult to intercept more than a small solid angle from the body. In order to determine a total body burden, the crystal and the subject must be some distance 
apart. As a consequence the counting efficiency becomes quite low. Even though the sensitivity is extremely high, on the order of a body burden of $5 \times 10^{-10}$ curie, the low efficiency necessitates long counting times. However, the counting times have been decreasing as the size of the crystal has been increased. Recently the counting times were about $30 \mathrm{~min}$ in contrast to the $2 \mathrm{hr}$ needed several years ago.

One method of increasing the counting efficiency, and consequently decreasing the counting time, is to surround the subject with the detector, in other words, a $4 \pi$ counting geometry. This is very expensive with NaI crystals but is feasible with a liquid scintillation counter. This is the system which we have used at Los Alamos. With this type of counter the efficiency is so high that one can obtain sensitivities similar to those of the NaI crystal with counting times of 2 min instead of $30 \mathrm{~min}$. This is an appreciable saving when one is making routine studies of large numbers of people. With a large detector of this type the background becomes relatively unimportant, thus eliminating elaborate shielding and the necessity for extremely pure component materials. Both samples and background rates are so high that good statistical precision is obtained in a very short time. An unusual degree of stability is required of the background, but because of the short counting times, this stability is essential only over periods of minutes rather than hours. This is illustrated graphically in Fig. 1. The percentage standard deviation is plotted against background count for various sampleto-background ratios and also for various sample rates. The counting time was $100 \mathrm{sec}$. Note that for a constant sample-to-background ratio the precision increases rapidly with increasing background. In this case the sample count is also increasing, so the statistics are better for a given period of time. In addition the plot shows that as the sample rate increases, the precision is almost independent of background. The background for the human counter is usually about $200 \mathrm{c} / \mathrm{s}$, and the average count per person is approximately $60 \mathrm{c} / \mathrm{s}$. With these counting rates the standard deviation does not vary more than from 1 to $1 \frac{1}{2}$ per cent with a background increase from 100 to $400 \mathrm{c} / \mathrm{s}$.

A schematic cross section of the counter $r^{3,4}$ is shown in Fig. 2. The subject is contained in a cylindrical cavity $\hat{b} \mathrm{ft}$ long and $18 \mathrm{in}$. in diameter. $\mathrm{He}$ is almost completely surrounded by the scintillation solution of 6 in. thickness (terphenyl plus POPOP in toluene). Scintillations are detected by 108 2-in.-diameter photomultiplier tubes arranged in rows along the outside wall of the counter. Figure 3 is a photograph of the counter rolled outside its 5-in. lead shield. The tank moves easily on ball-bearing rollers to facilitate tube installation, etc. The subject is introduced into the counting position by means of a canvas sling which rides in a loading trough. The cavity is closed at the end with a 2-in.-thick lead plug. Because of the 


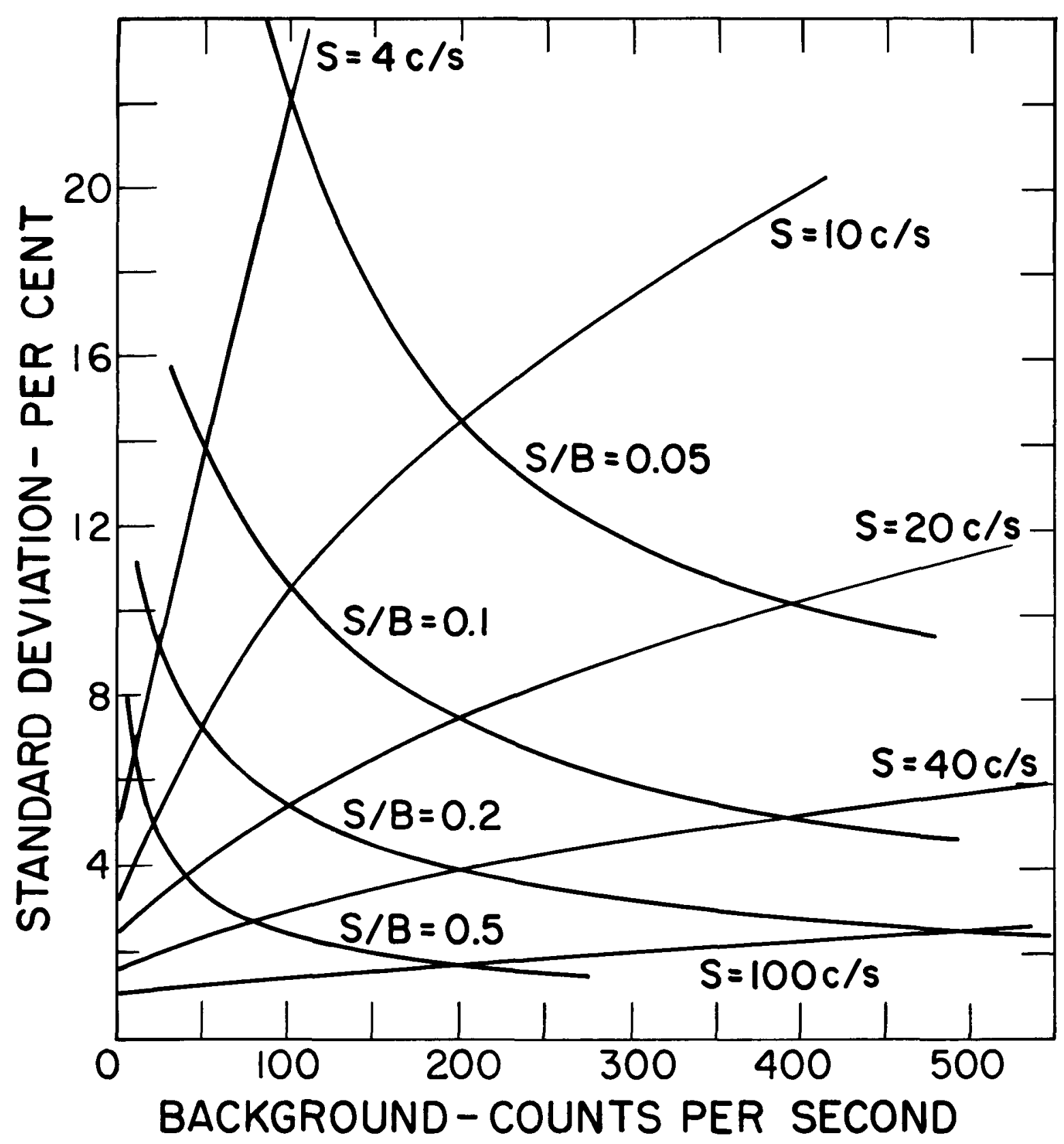

Fig. 1 Standard deviation as a function of sample : background ratios. 


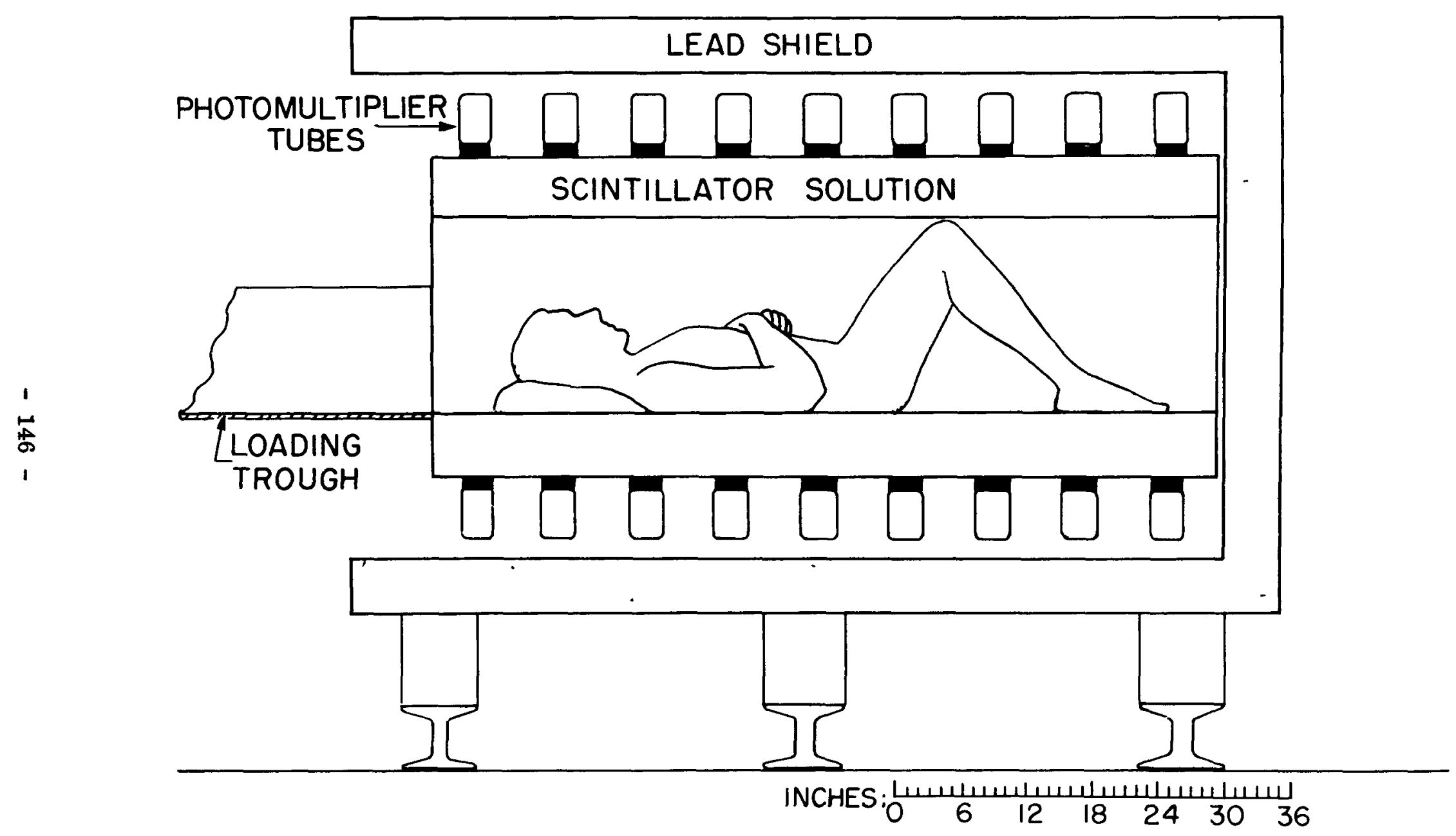

Fig. 2 Schematic cross section of the detector. 


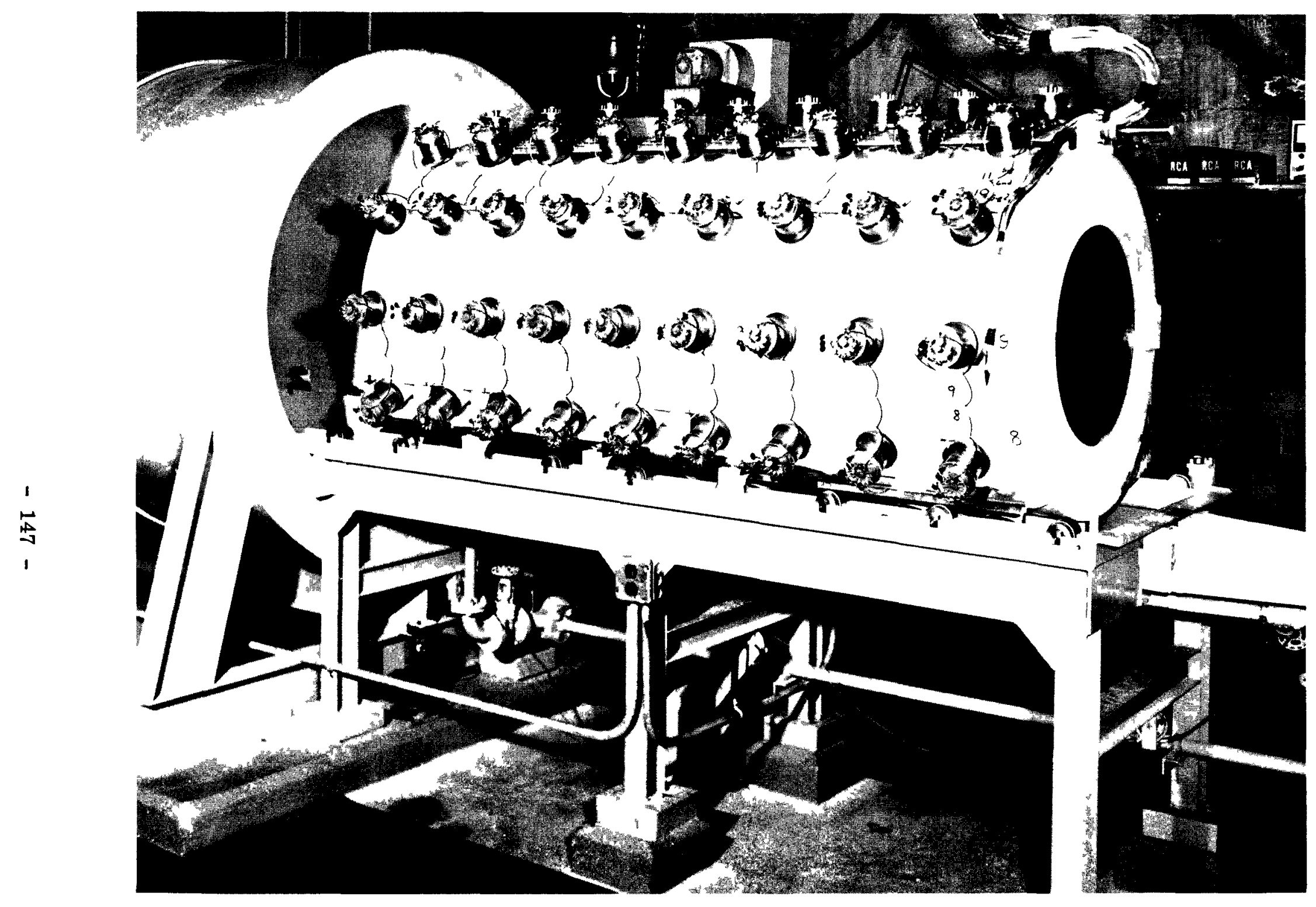

Fig. 3 Detector tank outside of lead shield. 
short counting time, ventilation is not a problem and has been handled adequately by air leakage around the plug. Atmospheric radon prevents the use of forced air.

The electronic equipment includes a special high-voltage control panel which provides individual switches for removing the high voltage from each photomultiplier tube, and individual variable resistors in series with each photomultiplier bleeder string. This makes it possible to test and balance each photomultiplier tube individually without removing the counter from the shield. Should a tube go bad during operation, it can be located and shut off within a few minutes. The counter will function quite well with as many as 10 tubes out, provided they are not too close together. The tubes are connected in parallel in two banks of 54 tubes each. A coincidence analyzer selects those pulses of the desired energy in one bank which are in coincidence with any pulse from the other bank. Two analyzers and scalers provide for the simultaneous determination of the activities in two different energy channels, 0.5 to $0.8 \mathrm{Mev}$ and 1.0 to $2.0 \mathrm{Mev}$.

Figure 4 shows how the sensitivity of the detector varies with a point source which was moved along the length of the counter. The solid curve is the calculated efficiency which would result from geometrical end-loss. The horizontal lines show the observed response to a $\mathrm{KCl}$ source. The agreement is quite good, thereby indicating that the only variation in counting efficiency is due to the geometry of the counter. Between 1 and $5 \mathrm{ft}$ the efficiency is more than 90 per cent.

The energy resolution obtained with the liquid scintillation detector is shown in Fig. 5, which shows the differential pulse-height spectra from $\mathrm{K}^{40}$ (1.4 Mev gamma) and $\mathrm{Co}^{60}$ (1.17 and $1.33 \mathrm{Mev}$ gammas). The $2.5 \mathrm{Mev}$ peak on the $\mathrm{Co}^{60}$ spectrum results from coincident pulses of both gamma rays. It is apparent that, while gamma rays differing in energy by a factor of 2 can be resolved, no exact determination of gamma-ray energy can be made since the half-widths of the pseudo-photopeaks are only 30 per cent at halfheight (because of multiple Compton scattering). This resolution compares very poorly with that obtained with the NaI crystal, but it is sufficient to discriminate between gamma emitters whose energies differ by an appreciable amount. The poor resolution is not necessarily inherent in the liquid scintillation detector, but is the result of the difficulty in getting the light out of the large volume and to the photomultiplier tube.

Before we used the instrument, we did a series of background studies to determine that the stability of the instrurnent was within statistical limits. An analysis of 67 determinations of $100-\mathrm{sec}$ backgrounds made during the course of 1 day is shown in Fig. 6. The results are plotted in terms of their deviation from the average background counting rate, $198.68 \mathrm{c} / \mathrm{s}$. The smooth curve is the expected Gaussian distribution of the events about the 


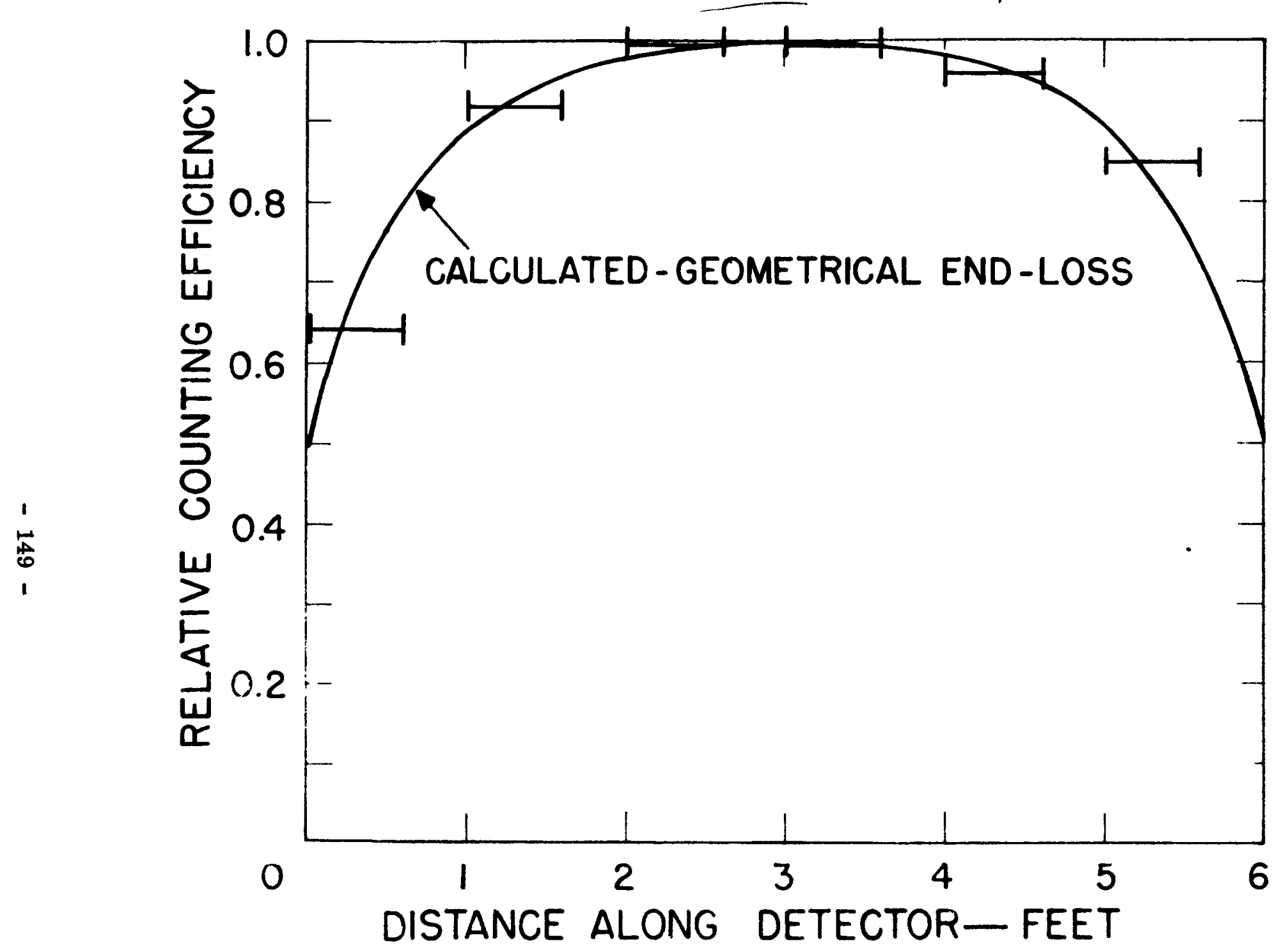

Fig. 4 Efficiencies obtained at several positions in the counter. 


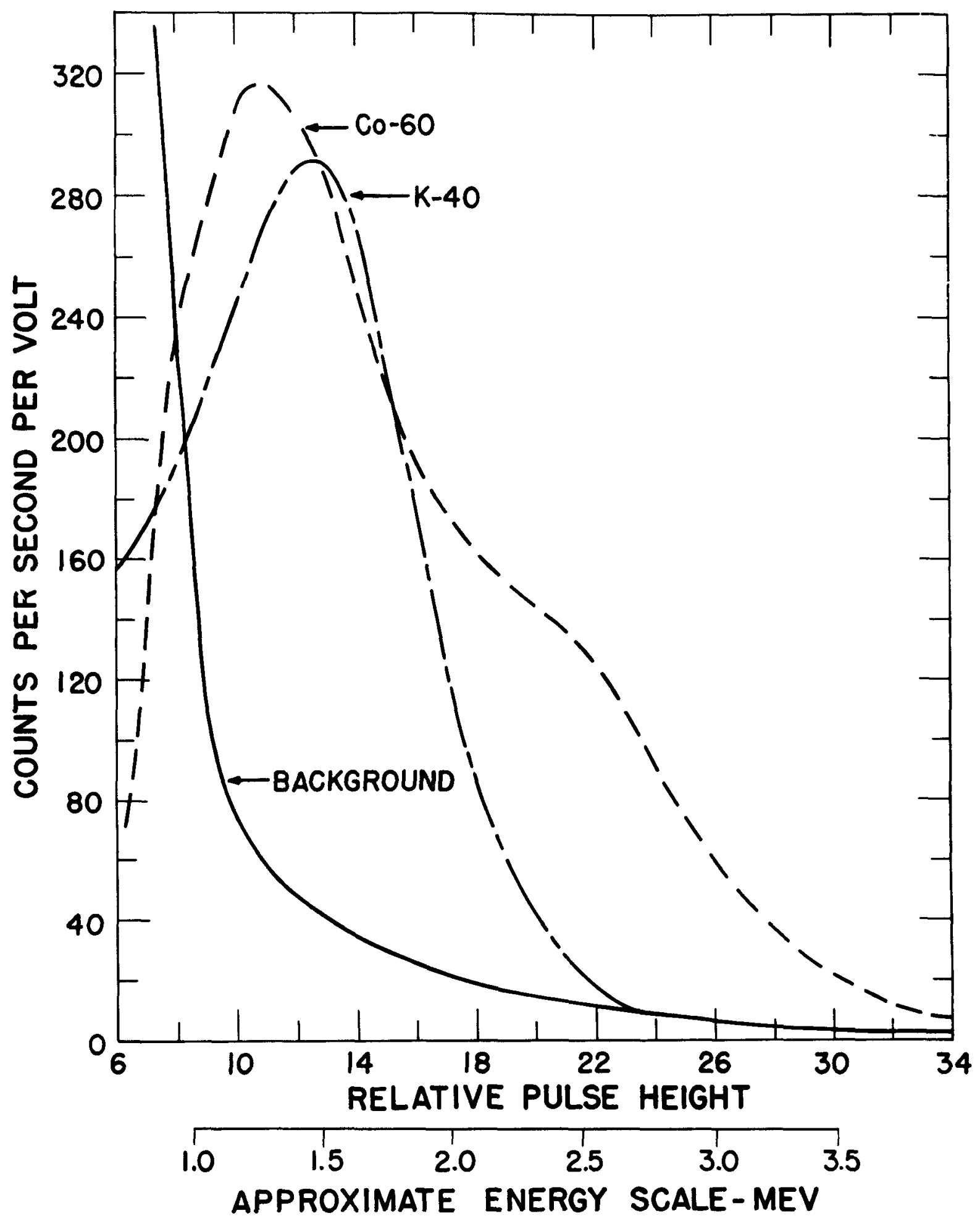

Fig. 5 Differential pulse-height spectra. 


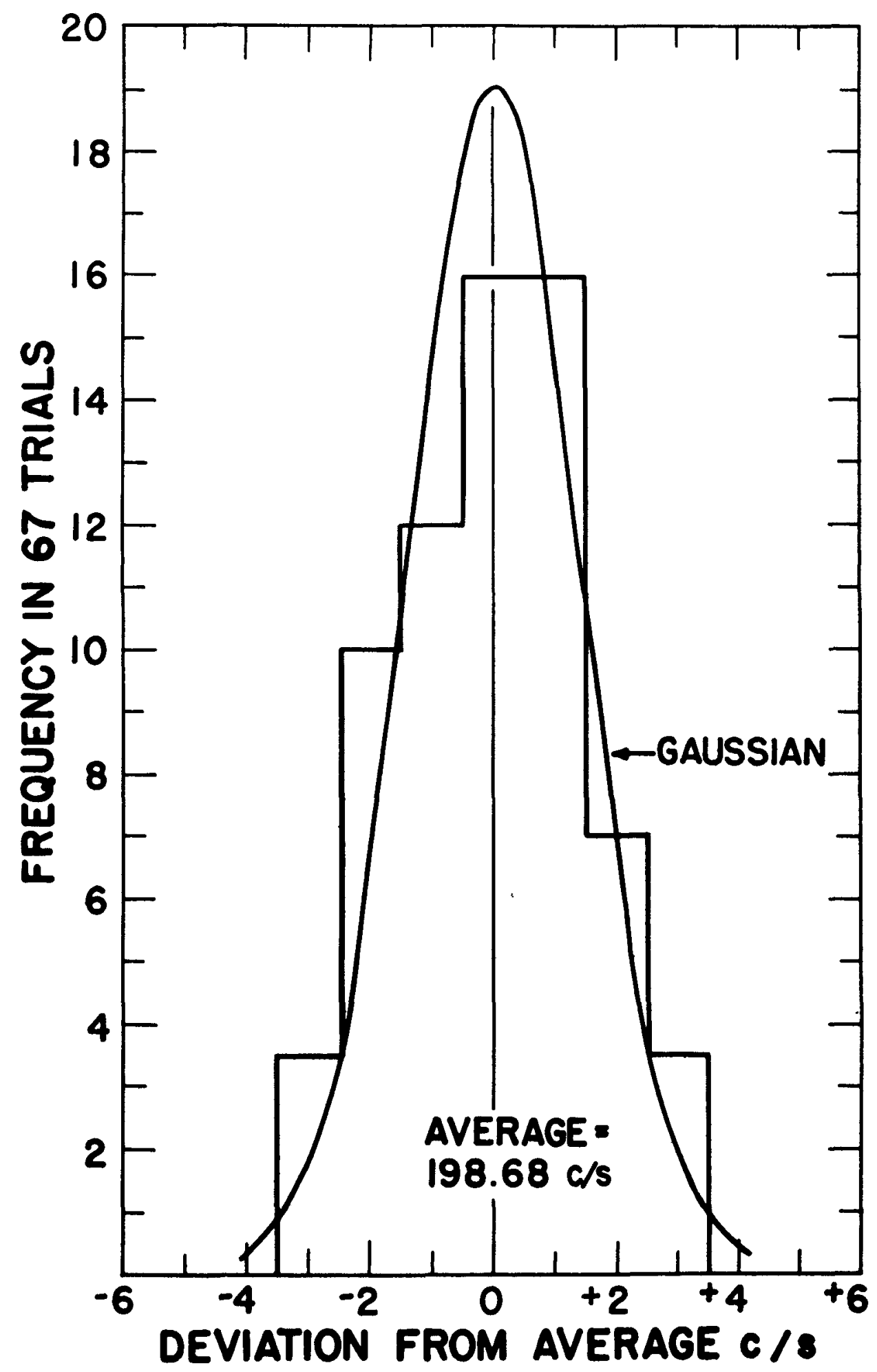

Fig. 6 Statistical distribution of 100-sec background counts. 
average, and the histogram shows the observed distribution. Under these conditions the standard error is approximately $1.4 \mathrm{c} / \mathrm{s}$, which is a bit better than a 1 per cent statistical precision. The results of a similar set of studies on stability are given in Table 1 . We used various counting times and also took some counts on a $\mathrm{KCl}$ standard, which increased the counting rate by a factor of 10 . The relative standard error, as computed from the observed scatter of the data about the average (last column), is compared with the error computed from counting statistics alone (fifth column). It is concluded that for a period of at least $2 \mathrm{hr}$, background is stable to at least 0.2 per cent; and for samples with 10 times background, the stability is 0.1 per cent over a 4 -hr period.

Table 1

STATISTICAL STABILITY

\section{Duration of Count, sec}

10

$40 \quad 140\left\{\begin{array}{l}35 \\ 35 \\ 35 \\ 35\end{array}\right.$

100

100

800

1400
Number of Runs

125

67

132

13

4

Counting
Time,
min

30

120

240

300

240

120

Average
Rate,
c/s

190.60

$\pm 4.4$

183.06

$\pm 2.1$

198.68

$$
\pm 1.4
$$

1563.0

$\pm 4.0$

1563.0

$\pm 1.4$

183.06

$\pm 0.36$
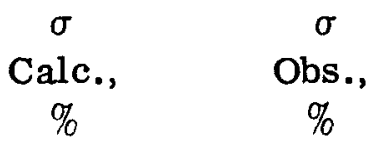

2.29

2.29

1.55

1.08

1.22

1.65

$\begin{array}{ll}0.71 & 0.75\end{array}$

0.25

0.27

1.37

0.11

0.09

0.11

0.20

0.21 
Natural $\mathrm{K}^{40}$ Determinations

We first directed our attention to the measurement of the natural $\mathrm{K}^{40}$ activity of people for two reasons: (1) it presents a good test of apparatus performance at the $0.01-\mu \mathrm{c}$ level, and (2) it constitues an inevitable "background" which must be subtracted to determine body contamination with other hard-gamma emitters. The results of the measurements of 82 subjects are shown in Fig. 7. We have plotted absolute disintegration rate, in the 1- to 2-Mev channels, against gross body weight. The considerable scatter of the data is due to variation in the muscle : fat ratio of the subjects since the potassium occurs principally in muscle and is absent in fat. These data show a pronounced scatter of the points to the right of a limiting line and a definite difference between males and females. As weight and age increase, people move away from this line with a decrease in potassium specific activity. The limiting line represents a body structure which includes a minimum amount of fat. The points that fall below the line are due to the addition of fat to the basic body structure. Using this line it is possible to predict the "fat-free," or "lean body," weight with considerable precision. This is of interest since the methods for determining body fat are difficult and time consuming. In addition, knowing the subject's height, weight, and age, one can predict, within limits, his natural potassium activity. With this information one can determine what fraction of the individual's total activity is due to a gamma-emitting contaminant in cases where the energies are not sufficiently different to resolve with our counter.

Correlation of potassium activity with fat-free body weight of a select group of these subjects (Fig. 8) indicated the amount of fat to be the principal factor causing variation in the apparent potassium content of the body. This corroborated our explanation of the variations shown in Fig. 7. We used two different methods of plotting each point in Fig. 8. The first is simply gross body weight versus gamma activity. In the second we determined the body water of the 14 subjects by tritium isotope dilution and computed the lean body weight from the body water using the equation

$$
\% \text { "fat-free" weight }=1.93 \times \% \text { body water }-39.9 \text {. }
$$

The equation involves certain physiological assumptions, but as an empirical relation the precision is sufficient for this study. These points are much more consistent than those obtained using the gross body weight. The standard deviation of the points about the average line is reduced from 17 to 6 per cent by this correlation. In routine monitoring it is usually sufficient to assume that an individual has $0.011 \mu \mathrm{c}$ of $\mathrm{K}^{40}$, which will be within 10 


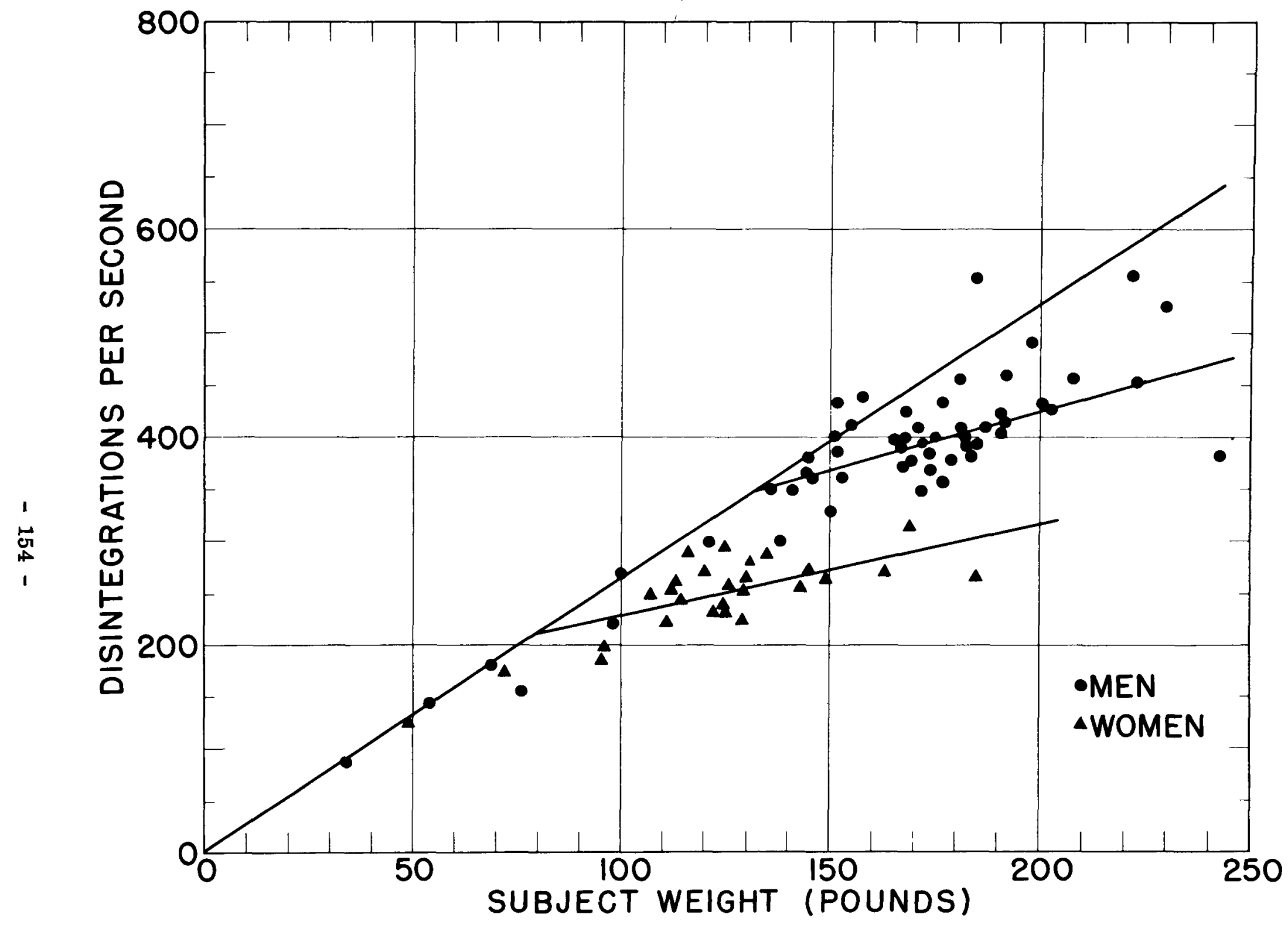

Fig. 7 Radioactivity of human subjects due to $\mathrm{K}^{40}$. 


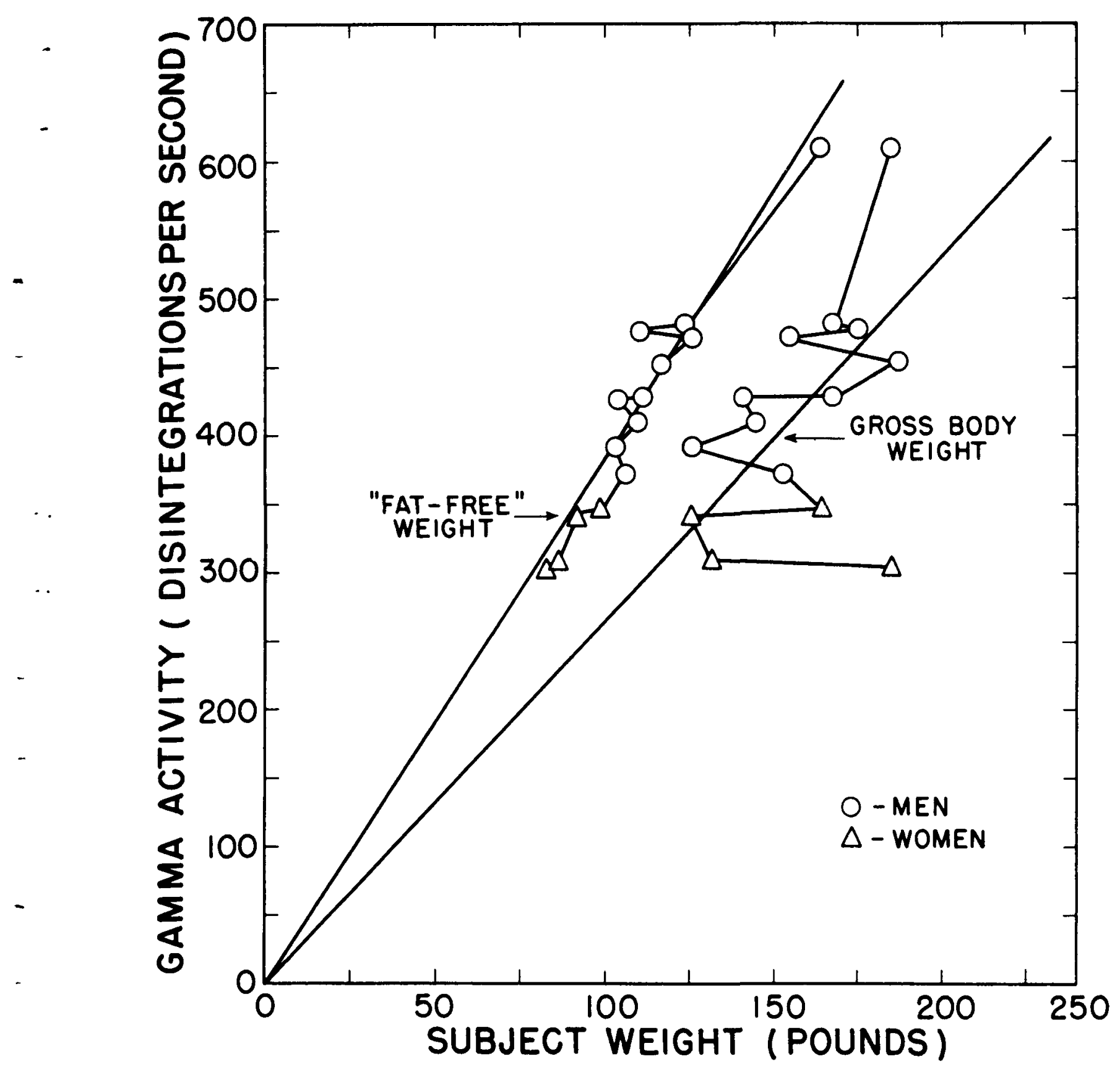

Fig. 8 Correlation of $\mathrm{K}^{40}$ activity with "fat-free" weight. 
per cent of the measured value, giving an uncertainty of $0.001 \mu \mathrm{c}$. Use of this correction will increase precision three- to fivefold.

A series of $\mathrm{K}^{40}$ determinations on two individuals over a 3-month period showed that the activity was constant within the 2 per cent statistical limits. Similar studies, shown in Fig. 9, were carried out from April to September of 1956. In this study the monitoring of the potassium activity was used to indicate the absence of any other hard-gamma-emitting material. The scattered high values during the period from June to September are probably due to surface contamination from tropospheric fallout during Operation Redwing. The sudden rise and fall of the values, also the fact that these apparently high $\mathrm{K}^{40}$ values were reduced to normal by bathing, indicates that they were due to surface contamination and do not represent an internal burden of some moderate or long-lived fission product.

\section{$\underline{\mathrm{Cs}^{137} \text { Studies }}$}

In the spring of 1956 Miller and Marinelli ${ }^{5,6}$ announced the identification of $\mathrm{Cs}^{137}$, a long-lived fission product (27-year half-life), in a number of people studied at Argonne. They were able to determine the isotope by its gamma energy and confirmed this by urinalyses for $\mathrm{Cs}^{137}$. We have confirmed these observations and found from 0.001 to $0.008 \mu \mathrm{c}$ of $\mathrm{Cs}^{137}$ in people. ${ }^{7}$ Since the $\mathrm{Cs}^{137}$ and $\mathrm{K}^{40}$ gammas differ in energy by a factor of 2 , differentiation is no problem. We have been measuring the $K^{40}$ in the $1-$ to 2-Mev channel and the $\mathrm{Cs}^{137}$ in the $0.5-$ to $0.8-\mathrm{Mev}$ channel. The first group contains only $\mathrm{K}^{40}$ activity and the second group contains the cesium plus a certain number of potassium counts. The fraction of the total potassium activity which appears in the lower channel can be determined easily with a potassium-sugar phantom, and corrections can be made easily for each individual.

During 1956 we made approximately 1500 cesium determinations on people. The average total body burden is apparently on the order of $0.005 \mu \mathrm{c}$, with a range of 0.001 to $0.008 \mu \mathrm{c}$. We have measured 186 people from 30 states and have found no geographical correlation. People who drink milk tend to have a higher cesium content than those who do not, but this does not always hold true. Eight subjects measured weekly from April 1 through September 10, 1956 (Fig. 10) showed no detectable change in $\mathrm{Cs}^{137}$ levels from April to June. In June the activity began to be erratic. This may be due to surface contamination, but from the data it appears that the cesium level is rising slightly for the rest of the period shown. This indicates that the cesium derives from slow stratospheric fallout with an ecological concentration, as does the $\mathrm{Sr}^{90}$ observed in people. The activity 


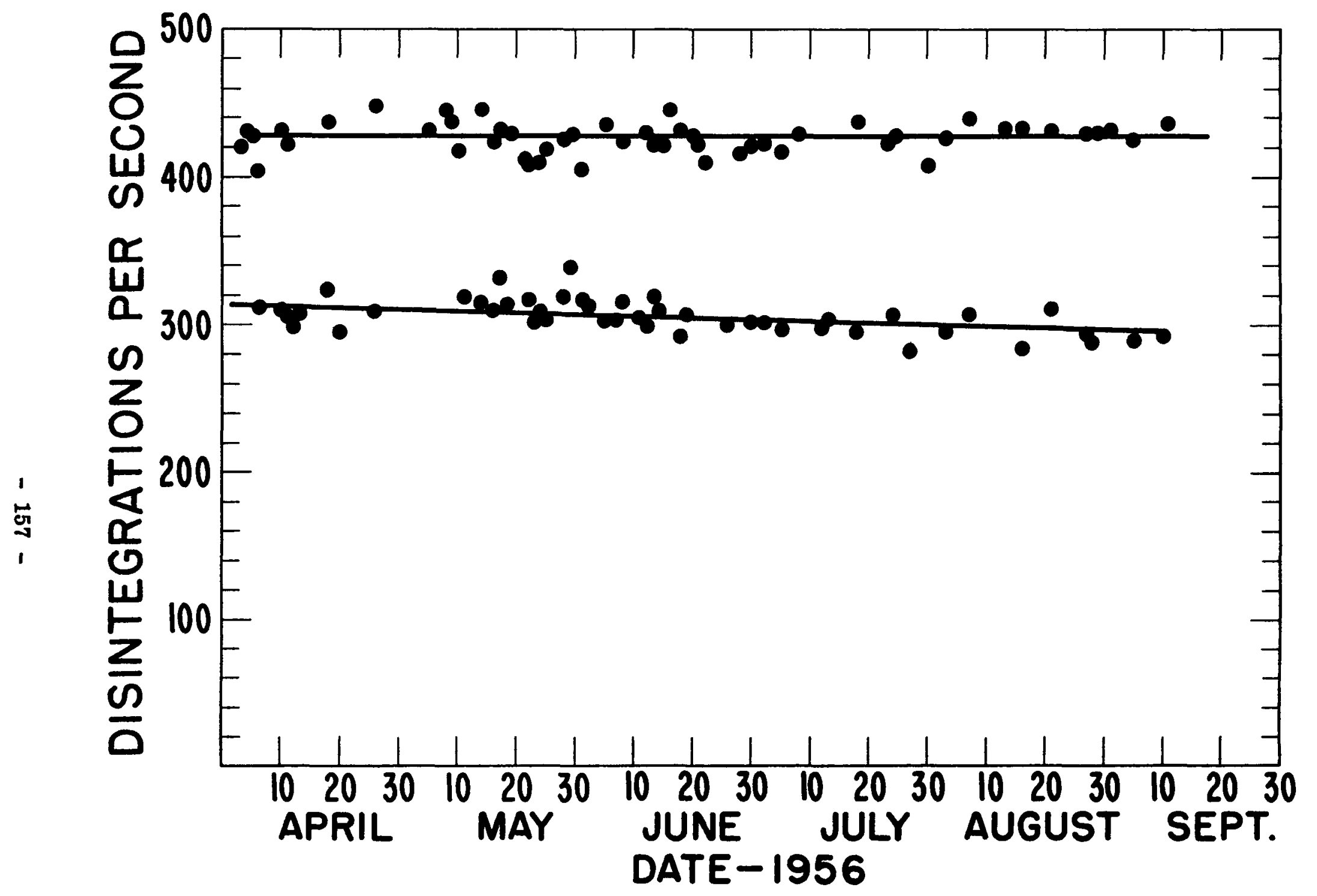

Fig. 9 The $\mathrm{K}^{40}$ activity of two subjects for 5 months during Operation Redwing. 


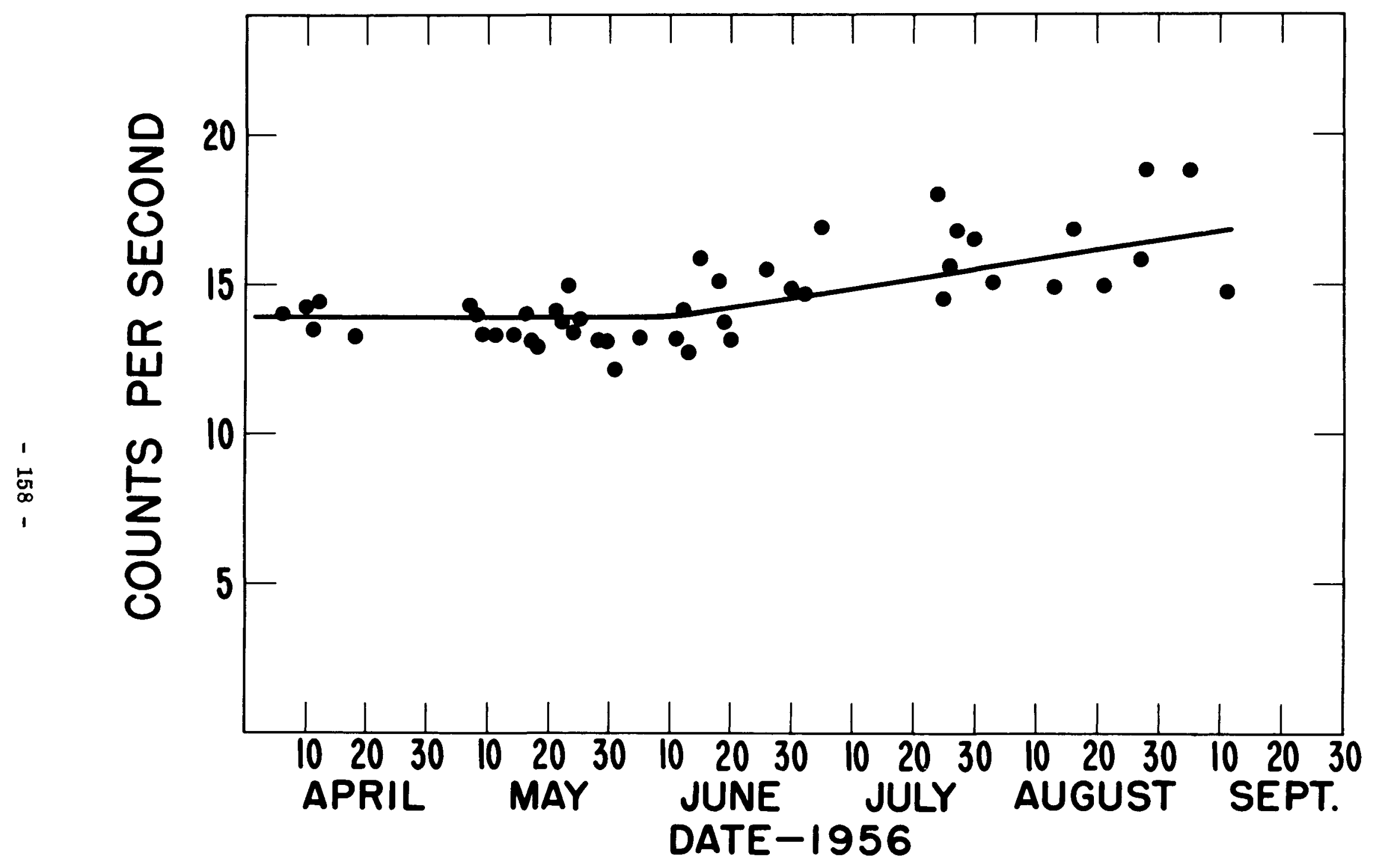

Fig. $10 \mathrm{Cs}^{137}$ levels for eight subjects during Operation Redwing. 
is assimilated by plants, vegetables, and grains and then is picked up by grazing animals and appears in meat and milk. This is a slow process with a latent period between the entrance of the isotope in the biosphere and its ingestion by man, so one would expect a gradual, not sudden, increase in the cesium activity of a population. Other moderately long-lived fission products, such as $\mathrm{Ru}^{106}, \mathrm{Zr}^{95}$, and $\mathrm{Ce}^{144}$ do not appear due to their low retention on ingestion.

While comparatively harmless itself, largely because of its short biological half-life of 144 days, ${ }^{8}$ the $\mathrm{Cs}^{137}$ level may provide a useful monitor of the rate of accumulation of the more dangerous $\mathrm{Sr}^{90}$. Direct conversion from one to the other is not possible because of their very different chemical behavior. However, the cesium results can yield valuable information on the rate and geographical distribution of fallout which will aid in the extrapolation of present $\mathrm{Sr}^{90}$ levels to the future. This would be very useful since the chemical determination of strontium is tedious and time-consuming. Attempts to count strontium by bremsstrahlung with the human counter have not been too successful.

We undertook a study of the $\mathbf{C s}^{137}$ levels in various foodstuffs to determine the more important ecological chains by which it enters the human body. Some of the preliminary results are given in Table 2.

Libby ${ }^{9}$ adopted the procedure of reporting $\mathrm{Sr}^{90}$ results as $\mathrm{Sr}^{90}: \mathrm{Ca}$ ratios because of the metabolic similarity of strontium and calcium, and to facilitate the comparison of different types of materials. Our Cs ${ }^{137}$ results are reported as the ratio of $\mathrm{Cs}^{137}: \mathrm{K}^{40}$ gamma disintegrations for similar reasons. While cesium is not a substitute for potassium, they are both alkali metals, and one can assume that their metabolisms are similar. Humans had, in the spring and summer of 1956, a Cs : $\mathrm{K}$ ratio of approximately 0.5 , and one can see that meats during that same period had a similar ratio. Pork, which comes from a nongrazing animal, is lower than lamb or beef. Other differences are apparent but the amount of data is, as yet, too limited to attempt detailed interpretation. Results on milk are shown in Fig. 11. The results are reported in terms of milk solids which, of course, have a very high potassium content, but the $\mathrm{Cs}: \mathrm{K}$ ratios are comparable to those found in meat. Data on the geographic distribution of the $\mathrm{Cs}^{137}$ : $\mathrm{K}^{40}$ ratio in milk are being collected and indicate that the distribution is essentially uniform. Two Australian milk samples are in agreement with the general U. S. average, lending support to the assumption that the general levels are derived from the stratospheric reservoir. A sample of American dried milk produced in 1942 showed no detectable $\mathrm{Cs}^{139}$ and the Cs : $\mathrm{K}$ ratio was less than 0.02 .

Based on the average diet of the people of the United States, using the Department of Agriculture figures ${ }^{10}$ for total food consumption, we estimated 
Table 2

RADIOACTIVITY IN FOODSTUFFS

Sample

Meats - Spring 1956

Beef rounds

Lamb, dressed carcass

Pork, fresh hams

Pork, loins

Meats - Winter 1956-1957

Beef, sirloins

Lamb, dressed carcass

Pork, loins

Pork, loins

Dried Blood

Illinois, April 1952

California, Winter 1956-1957

Minnesota, Winter 1956-1957

Texas, Winter 1956-1957

Flour - Spring 1956

High altitude wheat (Colorado)

Bleached, enriched (A)

Bleached, enriched (B)

Whole wheat, graham

Potatoes - Spring 1956

Colorado

Idaho

Vegetables - Spring 1956

Lettuce
Cabbage
Carrots

Fruits - Spring 1956

Tomatoes

Oranges

Grapefruit

Watermelon

Coffee - Spring 1956

$$
\mathrm{K}^{40}
$$

Specific Activity

(dis/sec/lb)

$\mathrm{Cs}^{137}: \mathrm{K}^{40}$

$\begin{array}{ll}3.84 & 0.53 \\ 3.83 & 0.81 \\ 3.52 & 0.30 \\ 3.23 & 0.19\end{array}$

2.75

0.15

3.67

0.16

0.10

0.07

3.26

9.2

$<0.07$

7.3

0.25

5.4

0.25

5.9

0.18

1.30

0.09

1.70

0.32

1.49

0.27

7.00

0.11

$7.82<0.06$

6.52

$<0.06$

2.34

$<0.07$

3.20

0.12

6.82

$<0.03$

3.81

0.03

2.10

0.38

3.30

0.25

3.75

$<0.03$

30.00

$<0.06$ 


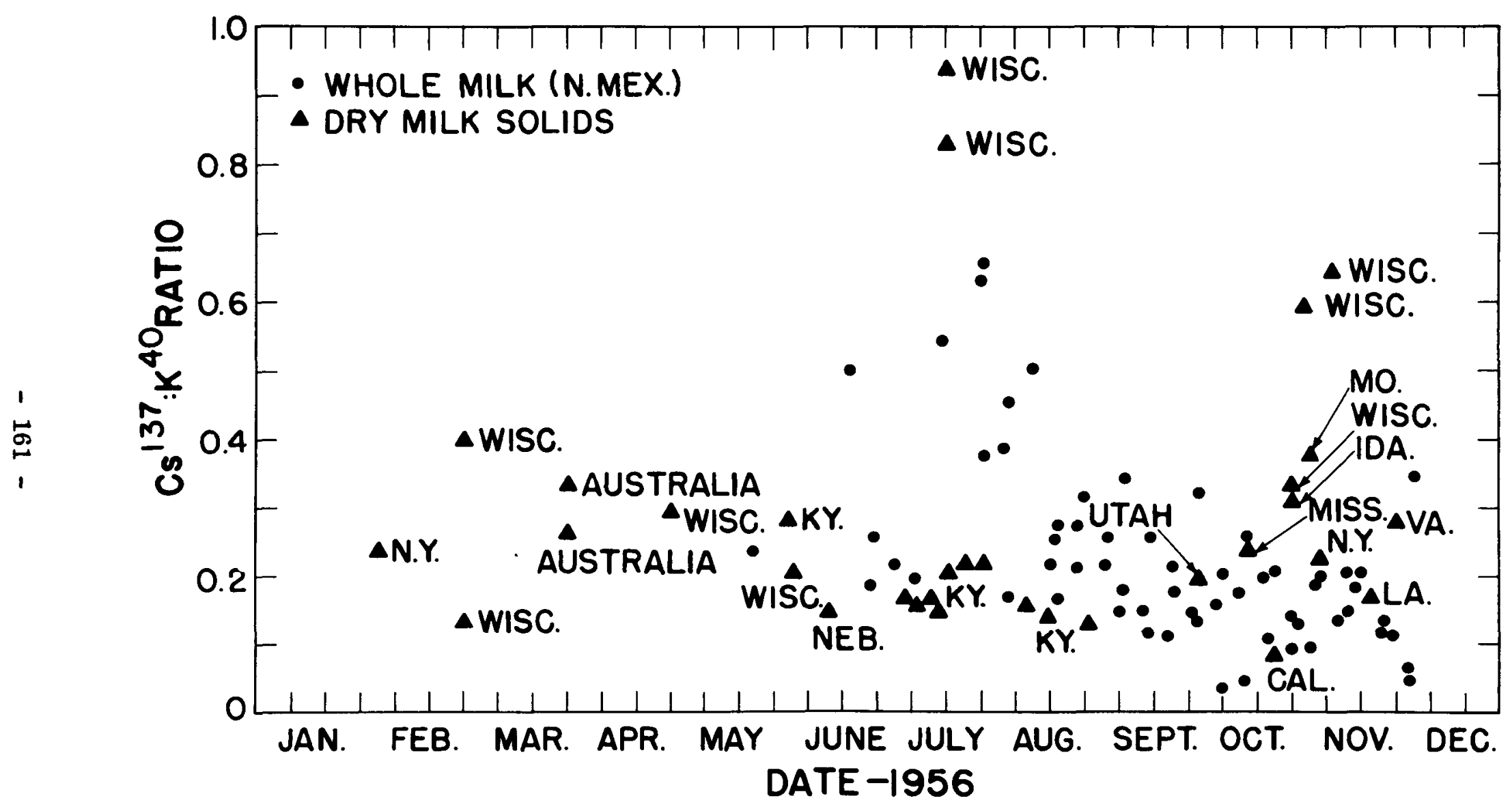

Fig. $11 \mathrm{Cs}^{137}: \mathrm{K}^{40}$ ratios for milk during 1956. 
that milk and milk products contribute about 50 per cent of the cesium ingested by humans. Meat contributes 25 per cent and the remaining 25 per cent comes from grain and vegetables. These figures are estimations since we have not been able to accumulate complete data as yet. It is a problem to obtain representative foodstuffs from various localities for adequate sampling, especially when one considers that we need 50- to 200-pound samples.

\section{Summary}

The Los Alamos human counter is a $4 \pi$ liquid scintillation detector capable of measuring the natural potassium content of the human body to a precision of 2 per cent in 100 seconds of counting time. Additional gamma contamination of as little as $0.0006 \mu \mathrm{c}$ can be detected in the same counting time if the gamma-ray energy exceeds $400 \mathrm{Kev}$. Measurements of the natural potassium have shown the human body to contain 0.28 per cent potassium on a "fat-free" weight basis independent of age, weight, or sex.

Cesium-137, presumably derived from stratospheric fallout from thermonuclear weapons tests, has been measured in people and in foodstuffs. The average amount of $\mathrm{Cs}^{137}$ in the human body is $0.005 \mu \mathrm{c}$ and appears to be derived principally from milk and meat.

The human counter has other potential applications. It may be possible to use it as a slow-neutron dosimeter. A whole-body or partial-body dose of thermal neutrons, as little as $0.3 \mathrm{rem}$, will produce enough $\mathrm{Na}^{24}$ activation in the body to give a measurable amount of gamma activity. The counters can be used to study cases of radium poisoning. The energies of the radium and potassium gammas cannot be resolved, but the radium can be determined by subtracting the predicted natural potassium activity. Fission product inhalation or ingestion can be detected with the instrument. In these cases one usually finds a high degree of external contamination. However, by a rigorous course of decontamination one can determine the body burden. 
References

1. Marinelli, L. D., Norris, W. P., Gustafson, P. F., and Speckman, T. W., "Transport of Radium Sulfate from the Lung and Its Elimination from the Human Body following Single Accidental Exposures," Radiology, 61, 903-915 (1953).

2. Marinelli, L. D., Miller, C. E., Gustafson, P. F., and Rowland, R. E., "The Quantitative Determination of Gamma-Ray Emitting Elements in Living Persons," Am. J. Roentgenol. and Radium Therapy and Nuclear Med., 73, 661-671 (1955).

3. Anderson, E. C., Schuch, R. L., Perrings, J. D., and Langham, W. H., "The Los Alamos Human Counter," Nucleonics, 14, 26-29 (1956).

4. Anderson, E. C., "In Vivo Gamma Measurements at Very Low Levels with $4 \pi$ Liquid Scintillation Detectors," IRE Trans. on Nuclear Sci., NS-3, 96-102 (1956).

5. Miller, C. E., and Marinelli, L. D., "The Gamma-Ray Spectrum of Normal Human Beings," Radiology, 66, 104-105 (1956).

6. Miller, C. E., and Marinelli, L. D., "Radium Toxicity, IV. Progress Report: Measurement of the $\gamma$-Ray Activities from the Human Body," Argonne National Laboratory Report ANL-5518, 52-56, January 1956.

7. Anderson, E. C., "Scintillation Counters, The Los Alamos Human Counter," Brit. J. Radiol., Supplement No. 7, 27-32 (1956).

8. Woodward, K. T., Richmond, C. R., and Langham, W. H., "Measurement of Retention and Excretion of Radioisotopes of the Alkali Metals by Mice and Rats, Using an Annular Liquid Scintillation Counter," Proc. of the Health Physics Soc., 79-88 (1956).

9. Libby, W. F., "Radioactive Fallout and Radioactive Strontium," Science, $123,657-660$ (1956).

10. United States Department of Agriculture Handbook No. 62, Supplement for 1954, U. S. Government Printing Office (1955). 